\title{
MAIZE NODAL ROOT GROWTH UNDER WATER DEFICITS
}

A Dissertation
presented to
the Faculty of the Graduate School
at the University of Missouri-Columbia
In Partial Fulfillment
of the Requirements for the Degree
Doctor of Philosophy
KARA J. RIGGS
by
Debert E. Sharp, Dissertation Supervisor
DECER 2016


The undersigned, appointed by the dean of the Graduate School, have examined the dissertation entitled

\section{MAIZE NODAL ROOT GROWTH UNDER WATER DEFICITS}

presented by Kara J. Riggs,

a candidate for the degree of Doctor of Philosophy, Plant Insect and Microbial Sciences, and hereby certify that, in their opinion, it is worthy of acceptance.

Professor Robert E. Sharp

Professor Walter Gassmann

Professor Felix Fritschi

Professor Abraham J. Koo 


\section{DEDICATION}

To my Lord and Savior Jesus Christ. This Ph.D. became the means by which I encountered God more closely than I had expected, and I am eternally grateful for this season. Thanks be to God, the author of resilience and redemption, who lived to die, who died to rise back to life, and who clothed perfection in rags and hid treasure in jars of clay. I hope you know how much He loves you, and how He died for you, and that His grace is as freely yours as it is mine through a shared faith and trust in Him as Lord.

Thank you to my amazing fiancé Widler Casy, who has supported and loved me throughout these intense past two years. Thank you for loving me for my quirks and my gifts, for your patience with my stubbornness and for allowing me to share those struggles with you. You inspire me to be a stronger, more courageous, thoughtful, hard-working, resilient, and dedicated woman of God. I love you with all my heart.

Thank you to my parents Bob and Lynne Riggs for believing in me and always loving me. Thank you Mom for driving me to OSU so many times, for encouraging my frequent questions as a young scientist, and for loving me exactly as I am. Dad thank you for being my constant support and encouragement, for teaching me compassion, and for spending your valuable time with me and our family despite the many demands on your time. Thank you both for teaching me to love the Lord and for setting a stellar example of integrity, love, courage, vulnerability, and loyalty. Thank you to my sister Dora and my 
brother Mark for loving and supporting me, and for always being eager to listen to and pray for me. I love you all so much.

Thank you to Dr. Greg Holliday who continued to encourage me to be myself, confront challenges, and focus on resilience practices that empowered me to persevere. I will always be grateful that you saw something special in me, and that you labeled it "creative" instead of "crazy," "strong" instead of "fearful," and "kind" instead of "weak."

Thank you to my graduate and non-traditional student group members. You have inspired and pushed me beyond what I thought I deserved. Here's to you, and I pray every graduate student finds the support they need through such encouraging strangers-turned-best friends. 


\section{ACKNOWLEDGEMENTS}

Thank you to Dr. Robert E. Sharp. You gave me the opportunity to grow as a scientist and as a person, challenging me to think independently and to motivate myself. I will always be thankful for the time I was in your lab. Thank you to all of the Sharp lab members who I had the honor of learning from and working with daily: Dr. Priya Voothuluru, Dante Smith, Hallie Thompson, Tyler Dowd, Jian Kang, and Justin Garnett.

Thank you to my committee members Dr. Walter Gassmann, Dr. Felix Fritschi, and Dr. Abe Koo for sharing your perspectives, helpful guidance, valuable time, and thoughtful comments. Thank you to Dr. Dale Blevins for your insight and advice in developing the aeration system and nutrient solution. Thank you to Dr. Steve Anderson for your helpful advice regarding the soil selection, testing, and drying. Thank you to Dr. Jeanne Mihail for your help and advice in my statistical analyses.

Thank you to the Life Sciences Fellowship Program and to the Division of Plant Sciences for funding my graduate program. I would also like to thank the faculty, staff, graduate, and undergraduate students of the Interdisciplinary Plant Group for creating a collaborative atmosphere that encouraged me to become a more creative and interdisciplinary scientist.

Thank you to Dr. Terrence Graham who gave me so much of his precious time and energy, entertained my many questions, and taught me how to think critically and systematically. I would not be here if you had not first entertained 
the awkward twelve year-old's questions about phytoalexins and continued to mentor me. I will always remember with great joy those years with you, Dr. Madge Graham, Dr. Serena Landini, Michelle Sinden, Dr. Jiye Cheng, and Ruth Russell in Kottmann Hall.

To Christy Copeland, Dr. Ken Schneeberger, Dr. Willi Meyers, and Dr. Anne Cafer, Fridah Mubichi, and Leah Sandler from CAFNR International Programs, thank you for giving me the interdisciplinary opportunities to build my skills. You not only taught me how to fish by empowering me with vital skill sets for agricultural development and teamwork, but you also taught me how to fly by dreaming of complex solutions reaching far beyond my plant scientist bench.

Thank you to farmers who feed the world, and thank you to the interdisciplinary, internationally collaborating scientists. The world was made infinitely better every time you leaned in, and you should be proud of your innovative spirit. Thank you. 


\section{TABLE OF CONTENTS}

ACKNOWLEDGEMENTS

LIST OF FIGURES

vii

ABSTRACT ix

\section{Chapter 1}

LITERATURE REVIEW: MAIZE NODAL ROOT GROWTH RESPONSE TO

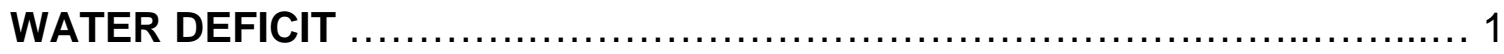

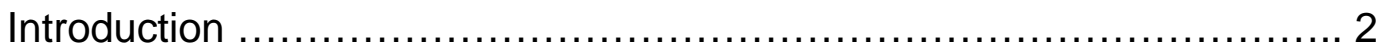

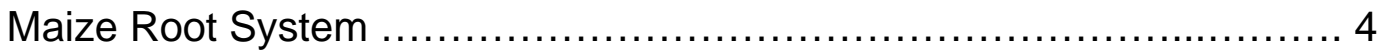

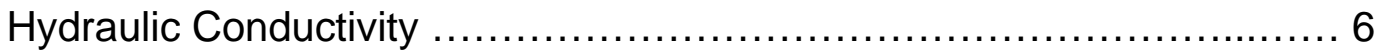

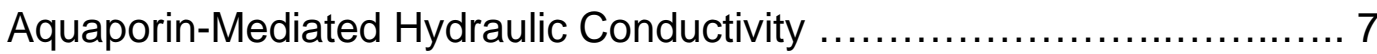

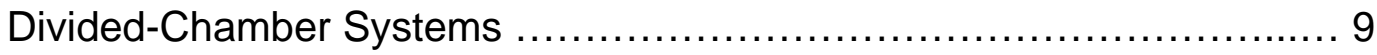

Taking into Account Plant Water Status .............................. 11

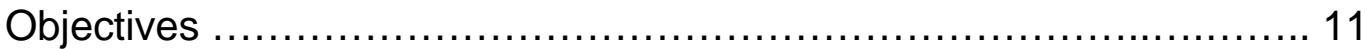

\section{Chapter 2}

DEVELOPMENT OF A NEW DIVIDED-CHAMBER MODEL SYSTEM FOR THE CHARACTERIZATION OF MAIZE NODAL ROOT GROWTH RESPONSE TO

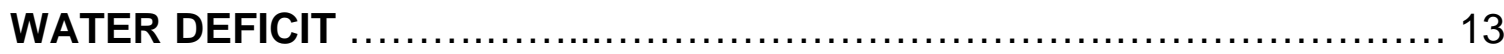

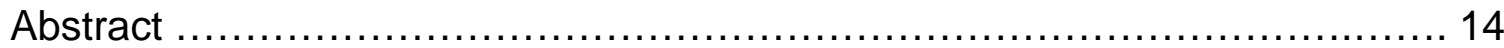

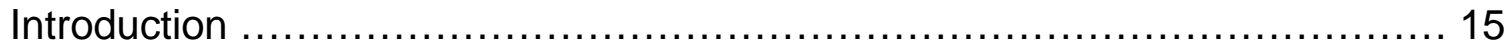

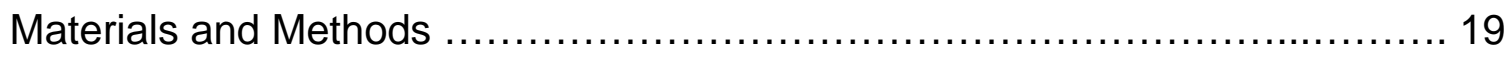

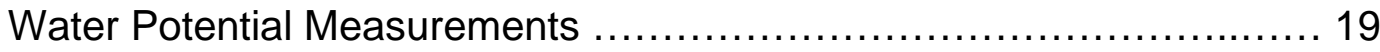

Growth Media and Preparation ...................................... 19

Growth of Plant Materials and Imposition of Water Stress ............... 21

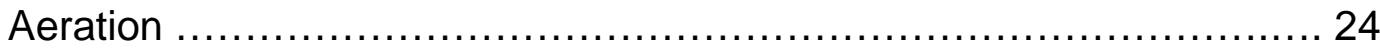




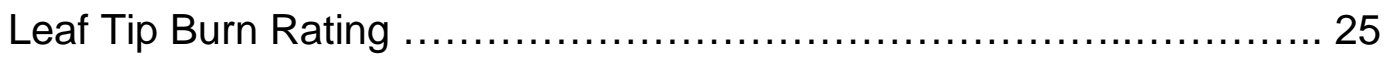

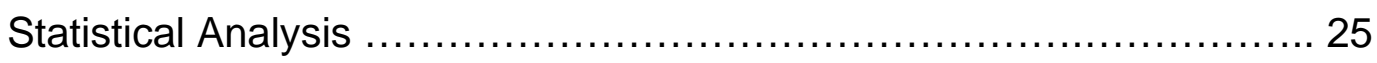

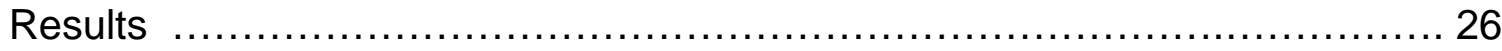

Peat-Based Media Supports Nodal Root Growth ....................... 26

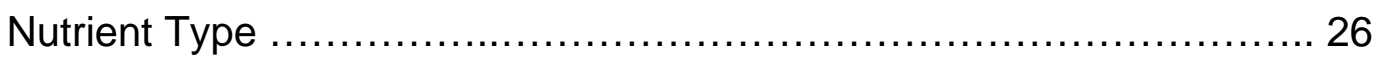

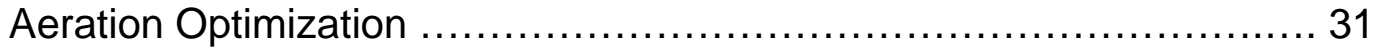

Steady-State Nodal Root Growth Rate .............................. 36

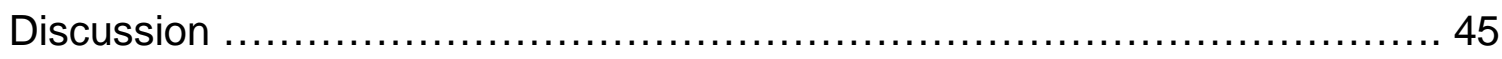

\section{Chapter 3}

MAIZE NODAL ROOT GROWTH RESPONSE TO WATER DEFICIT IN THE

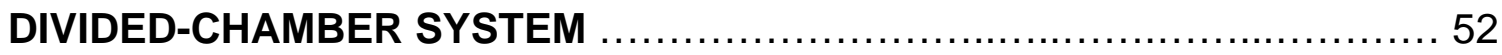

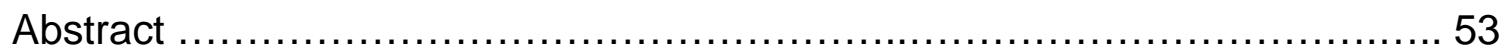

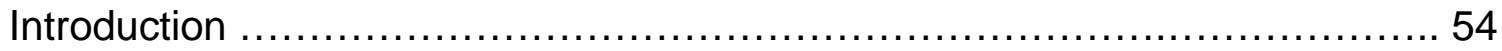

Root Tip Water Potentials: Limits to Hydration .......................... 55

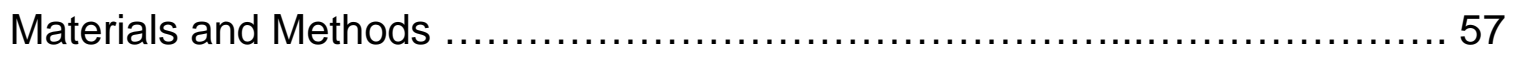

Growth of Plant Materials ….......................................... 57

Water potential measurements ..................................... 57

Measurement of Root Length and Shoot Growth ....................... 58

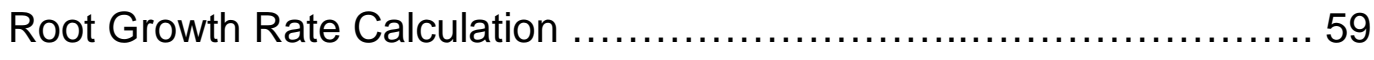

Statistical Analyses ................................................ 59

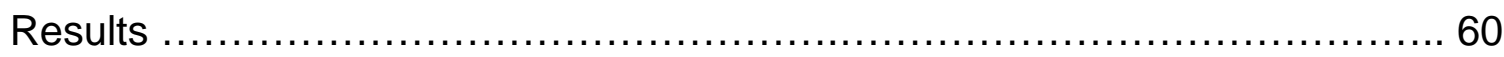

Nodal Root Growth is Sensitive to a Mild Drop in Soil Water Potential ... 60

Nodal root axes length is maintained between moderate and severe water

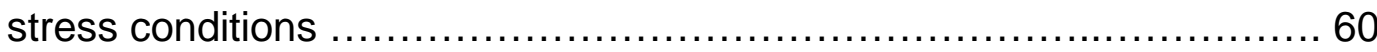

Root Tip Water Potential Corresponds with Growth Differences in Severe

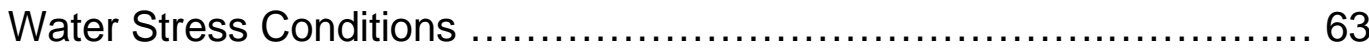

Relationship Between Soil Water Potential and Root Tip Water Potential 68

Growth Rate Corresponds to Root Tip Water Potential .................. 73 
Water Potential Gradients into the Root Tip .......................... 80

Shoot Growth Declines with Low Soil Water Potential in the Outer Chamber

Seminal and Primary Root System Growth Increases with Low Soil Water Potential in the Outer Chamber 87

Nodal Lateral Root Growth Decreases with Low Soil Water Potential in the Outer Chamber 93

Discussion 93

Hydraulic Conductivity of Nodal Axes ................................. 98

Possible Suberization Response ..................................... 101

Carbon Partitioning and Plasticity of the Root System ................... 102

Leaf Growth in the Divided-Chamber System ......................... 104

\section{Chapter 4}

FUTURE STUDIES AND IMPLICATIONS FOR TRANSLATIONAL RESEARCH

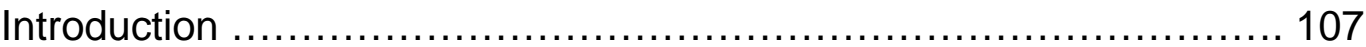

Future Anatomical Studies ............................................ 108

Future Hydraulic Conductivity Studies ................................ 109

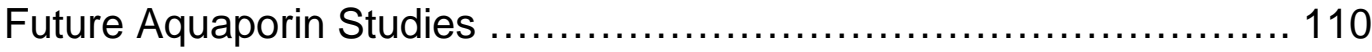

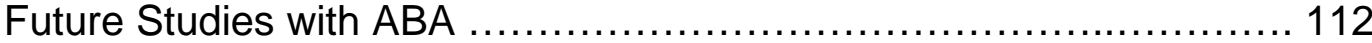

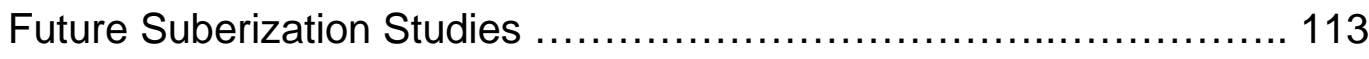

From the Lab to the Field ......................................... 114

\section{Appendix 1}

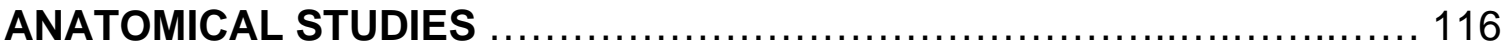

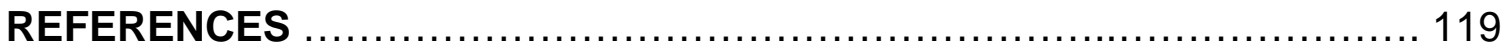

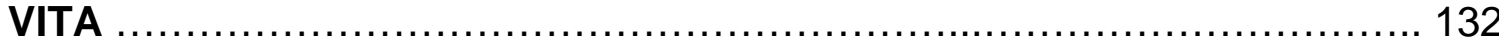




\section{LIST OF FIGURES}

Figure

Page

2-1 Design of the maize divided-chamber model system .........

2-2 B73 and FR697 shoots at the developmental stage for excavation

2-3 Root elongation rate of Node 1 roots in the dividedchamber system

2-4 Total seminal root system length and total nodal root system length of B73 plants

2-7 Dry weight of root and shoot tissue and root to shoot dry weight ratio

2-8 Diagram of the divided-chamber aeration system

2-9 Leaf tip burn rating of B73 plants

2-10 Dry weight of root and shoot tissue and root to shoot dry weight ratio

2-12 Diagram of the divided-chamber system

2-13 Leaf tip burn rating of B73 plants

2-14 Leaf tip burn rating of B73 plants

2-15 Root growth rate of Node 1 roots 
3-3 Nodal root axis length

3-4 Nodal root tip water potential as a function of soil water potential

3-5 Nodal root tip water potential as a function of soil water potential

3-6 Nodal root tip water potential as a function of soil water potential

3-7 Nodal root axis length as a function of nodal root tip water and soil water potential

3-8 Nodal root growth rate as a function of soil water potential and nodal root tip water potential

3-9 Nodal root growth rate as a function of soil water potential and nodal root tip water potential

3-10 Tissue water potentials of nodal root tips and mature nodal root axes

3-11 Tissue water potentials of nodal root tips and mature nodal root axes

3-12 Tissue water potentials of nodal root tips and mature nodal root axes

3-13 Leaf area as a function of soil water potential .

Stem height

Seminal root length

Nodal lateral root length .... 


\title{
MAIZE NODAL ROOT GROWTH UNDER WATER DEFICITS
}

\author{
Kara J. Riggs \\ Dr. Robert E. Sharp, Dissertation Supervisor
}

\begin{abstract}
The nodal root system is critical for the development of the mature root system in maize (Zea mays L.) and other grasses. Under drought conditions, nodal root axes may need to grow through surface soil that is dry, hard, and hot. These roots are known to have a superior ability to continue elongation at low water potentials relative to other organs of the plant, but the physiology of this response has been little studied. The objective of this study was to develop an experimental system that models the field situation in which upper soil layers dry, to enable studies of nodal root growth regulation under water deficit conditions. A divided-chamber experimental system was developed to allow the growth of maize primary and seminal root systems in well-watered conditions while the nodal root system is exposed to precise conditions of low soil water potential. The divided-chamber system was used to characterize nodal root growth responses to a range of soil water potentials under steady-state and reproducible conditions. Two contrasting genotypes, selected for differences in root growth response to water stress based on a previous study of the primary root, displayed similarly sensitive growth responses to $-0.3 \mathrm{MPa}$ soil, but different capacities to maintain high root tip water potential corresponding with different growth responses at lower soil water potentials. Both genotypes maintained relatively high nodal root tip water potentials in -2.0 MPa soil, despite the
\end{abstract}


decreased soil water potential, suggesting a stress-induced response that enhances water transport to the root tip. The difference in high tissue water potential maintenance was seen not only between the contrasting genotypes but also between the first two developmental nodes of roots. The divided-chamber system provides a powerful experimental approach to investigate the physiological mechanisms regulating nodal root growth responses to adverse soil conditions. Future studies may include measurements of hydraulic conductivity, anatomical characterization of vascular elements near the growth zone, aquaporin content and activity, and suberin deposition in response to low soil water potentials. 


\title{
CHAPTER 1
}

Literature review: Maize nodal root growth response to water

\author{
deficit
}




\section{Introduction}

Drought is one of the major limitations to agriculture world-wide (Boyer, 1982). During the drought of 2012 in the United States, $80 \%$ of all agricultural land was affected, creating large crop losses due to drought (Wallander et al., 2013; Boyer et al., 2013). As climates continue to change and the amount of arable land continues to decline, the efficient use of land and water resources will become even more crucial to feed a growing population (Bagley et al., 2015; The Royal Society, 2009). Maize is a staple crop in the US agricultural economy, with US farmers planting over 90 million acres of maize annually (USDA, 2016).

The yield of maize is particularly sensitive to drought stress (Lobell et al., 2014), particularly during reproduction and grain filling (Bolanos and Edmeades, 1996; Boyer, 1982). If the root system of a plant perceives soil drying, the roots transfer a signal to the shoots to close stomata (Dodd, 2003; Sobeih et al., 2004). This response decreases the rate of water loss due to transpiration and conserves the amount of water in the soil for plant growth (Kang and Zhang, 2004). The practice of allowing partial root zone drying in rice has proved to be beneficial for water management in China (Du et al., 2015) in addition to no loss in yields (Davies et al., 2011). However, the yield may be affected by this conservation of water if the photosynthetic rate of the plant decreases substantially, as the diffusion of carbon dioxide into the leaf slows and becomes rate-limiting to the plant (Lawlor and Cornic, 2002). As the rate of photosynthesis decreases, a decreasing level of sucrose is perceived in the ovary, and the ovary aborts. Although this response can be prevented with iso-osmotic sucrose 
infusions near the ovary (McLaughlin and Boyer, 2004), it cannot be reversed, dramatically affecting the yield of the plant.

To develop more drought tolerant crops, a basic understanding of key physiological mechanisms underlying drought tolerance and adaptation is needed. The mechanisms underlying plant responses to drought include diverse chemical and hydraulic signals as previously reviewed in Chaves et al., 2003. Although often overlooked as "the hidden half" (Eshel and Beeckman, 2013), the root apices have been attributed as the brain of the plant, due to their importance in perceiving and creating signaling cascades that affect the entire plant's health and survival (Baluska et al., 2004).

The root system is also vital for the continued acquisition of water and nutrients for the growing plant. Previous studies have shown that dryland plants develop deeper rooting systems than irrigated plants (Weaver, 1926). An increase in rooting depth can correspond with a yield increase in rain-fed wheat production (Lilley and Kirkegaard, 2011). Deeper rooting may increase the plant's chance of survival in drought situations, as it is able to access more water resources in an increased volume of soil resources within the soil profile (Hochholdinger, 2016; Lynch, 2013). It has been shown that fewer but longer maize nodal axes can increase water access compared to less intensive rooting genotypes under water deficit conditions (Gao and Lynch, 2016). Improvements in root growth maintenance under water stress will allow the plant to access the water and nutrient resources deeper in the soil profile and continue to support the development of the plant in water-limited conditions. 


\section{Maize root system}

The root system of maize is comprised of three classes of roots: primary, seminal, and nodal roots (Hochholdinger and Tuberosa, 2009). The primary root is present in the embryo and is the first root to emerge upon germination of the seed. Both the primary root and seminal root systems support the growth and development of the seedling (Fusseder, 1987). The mechanisms of maize primary root growth maintenance at low water potentials have been studied extensively in previous studies. Cell wall loosening (Wu et al., 1996; Wu et al. 2001), plasma membrane proteins (Zhu et al., 2007; Voothuluru et al., 2016), and reactive oxygen species (Voothuluru and Sharp, 2013) all play crucial roles in optimizing growth in water deficit conditions in the maize primary root. Through detailed kinematic studies, the preferential growth maintenance of the apical tip (region 1, 0-3 mm starting at the root cap junction), has been demonstrated in multiple genotypes (Yamaguchi and Sharp, 2010). Drought sensitive lines tend to have shorter growth zones, which correspond to slower growth rates (Sharp et al., 1988; Spollen et al., 1993).

Maize nodal root axes are initiated from a zone of highly compressed nodes at the base of the shoot called the crown (Hoppe et al., 1986). During seedling establishment, the nodal roots begin to develop and later comprise the majority of the mature root system of maize (Hochholdinger et al., 2004). The first nodes of roots emerge below ground, while the last nodes to form emerge above the soil surface. These later-forming nodal roots are also called crown or brace roots because of their added function of mechanical support for the maize plant 
(Hochholdinger et al., 2004). However, the formation of early nodes of roots is also crucial for the mechanical stability of the young shoot. If the first few nodes fail to form, in a condition called rootless corn syndrome, the young plant will lodge, possibly breaking the mesocotyl attaching the shoot to the seminal and primary root systems, killing the plant (Elmore and Abendroth, 2007).

Although the establishment of the nodal root system is essential to the health and survival of the maize plant, little information is known about the physiological response of nodal roots to water deficit. Under water stress, nodal roots have a superior ability to maintain growth at water potentials that are growth-inhibitory to other parts of the plant (Westgate and Boyer, 1985).

Nodal root growth under water stress can be maintained by osmotic adjustment, in which a tissue lowers its osmotic potential to draw in water and thereby maintain turgor pressure for cell expansion. Nodal roots are capable of exceptional osmotic adjustment, which may help explain why they are able to maintain growth at low water potentials (Sharp and Davies, 1979; Westgate and Boyer, 1985). An extreme case of growth maintenance can be observed when nodal roots penetrate the dry, hard, and hot surface soil in the field to grow toward water resources deeper in the soil profile. Because nodal roots arise adventitiously from the compressed nodes at the base of the maize stalk, in the surface soil layer (Hoppe et al., 1986), nodal roots must be able to maintain growth in sub-optimal conditions. 


\section{Hydraulic conductivity}

Hydraulic conductivity, the ease of water transport through the plant, has a significant impact on the level of water stress the plant experiences, or the water potential of a tissue. Water moves passively through living tissues down thermodynamic water potential gradients. As water evaporates through stomata, it creates a negative pressure (tension) in the xylem elements, pulling water out into the atmosphere and throughout the plant from its original source in the soil. The stomata, which control transpiration rate, account for about half of the hydraulic resistance in the plant (Javot and Maurel, 2002).

Water moves through plants in a sum or differing combinations of the following three pathways: in the apoplast (through the cell walls), in the symplast (through plasmodesmata), and in the trans-cellular path (across membranes). The degree to which a given pathway is favored depends on genotype and the environmental conditions (Javot and Maurel, 2002). As transpiration rate slows, osmotic forces predominate and water movement occurs intercellularly (Steudle and Peterson, 1998).

While some reports show a majority portion of water moving in the maize root symplast and transcellular paths (Canny and Huang, 1994), other studies have suggested a majority of water movement in the apoplast, particularly during times of high transpiration rate (Steudle and Peterson, 1998). A possible explanation of this discrepancy may be that the aeroponic culture in which maize plants were grown by Canny and Huang, which could have induced a thickening of the exodermis. In cell layers containing an exodermis, where apoplastic water 
movement is excluded, aquaporins are found in relatively higher densities to increase the hydraulic conductivity of the transcellular and symplastic pathways. Water movement meets the highest resistance in those tissues without differentiated vascular elements. At a certain proximity to the root tip, the meristem must be supplied water and solutes by radial transport through neighboring cells, because the xylem and sieve cells have not yet differentiated (Pritchard et al., 1996).

The direction of water movement in the root tip depends on the water potential of the soil and the tissue. In well-watered conditions, radial transport across the cells external to vessel elements is estimated to account for $70-90 \%$ of the resistance to water movement across living tissue in the roots (Javot and Maurel, 2002). In the unique situation when roots have grown through air, phloem has been sufficient to supply $45 \%$ of the water required for root growth (Boyer et al., 2010). Previous studies (Sharp and Davies, 1979) have shown a drastic drop in water potential at the root tip under water deficit conditions. If the tissue immediately behind the tip is of a higher water potential, water will flow radially from the nearest vascular element into the root tip.

\section{Aquaporin-mediated hydraulic conductivity}

Aquaporins are multifunctional channels that not only transport water but can also selectively transport various compounds, depending on the residues surrounding the channel pore opening (Chaumont and Tyerman, 2014). Aquaporins comprise a large and diverse gene family in plants, accounting for 
about 30-70 protein homologs in monocots like maize alone. Aquaporins are classified into five subgroups based mostly on their localization and sequence similarity: plasma membrane intrinsic proteins (PIPs), tonoplast intrinsic proteins (TIPs), Nodulin26-like intrinsic proteins (NIPs), small basic intrinsic proteins (SIPs), and the $\mathrm{X}$ intrinsic proteins (XIPs).

PIPs and TIPs are involved in the radial transport of water through the root cylinder and in osmoregulation, making them aquaporin subgroups of interest for drought studies. PIPs and TIPs are also expressed more abundantly in roots than in leaves, most likely due to the necessary trans-cellular movement that is required in roots around apoplastic barriers like the Casparian strips in the exodermis and endodermis (Chaumont and Tyerman, 2014).

Aquaporins are regulated in various ways. In short term responses, rapid transcriptional and post-translational modifications are involved, including the following described modifications: phosphorylation, methylation, ubiquitination, deamidation, disulfide bond formation, and protonation (Chaumont and Tyerman, 2014). These processes have various effects on aquaporin trafficking and gating.

In the maize primary root, aquaporin protein content is responsive to water stress in specific physiologically relevant regions. In the maize genotype B73, the aquaporin gene ZmTIP4-1 was preferentially expressed in the pericycle versus non-pericycle cells (Dembinsky et al., 2007). In recent studies on maize root tips (FR697), several aquaporins were detected under water stress using a plasmamembrane enrichment protocol. Most notably, the aquaporins ZmTIP2-1 and 
ZmPIP1-2 increase in abundance in the apical-most portion of the tip where growth is maintained (Voothuluru et al., 2016).

Hormones such as abscisic acid (ABA) affect the growth rate of roots under water stress and may also facilitate the regulation of the water status of a tissue through aquaporin signaling. ABA has been shown to positively affect root hydraulic conductivity. Exogenous ABA treatment and ABA overproducing lines showed an increase in hydraulic conductivity of the root system (Thompson et al., 2007). By use of a cell specific pressure probe, Hose et al. showed an increase in hydraulic conductivity in excised maize roots treated with ABA, the effects of which were most pronounced in the cortical cell layers (Hose et al., 2000). The group postulated ABA affects water channels in the symplast, and these hypotheses were supported in later studies (Parent et al., 2009).

Maize transgenic lines negatively affected in 9-cis-epoxycarotenoid dioxygenase (NCED) expression, which catalyzes the first committed step in ABA biosynthesis, showed decreased expression of PIP genes and decreased PIP protein content (Parent et al., 2009). The resulting hydraulic conductivities of the antisense lines were greatly reduced. Sense plants showed increased hydraulic conductivity. This change in conductivity was attributed to an increase in aquaporin activity, because the difference could be abolished by hydrogen peroxide treatment, blocking aquaporin water transport (Parent et al., 2009).

\section{Divided-chamber systems}

Because of the extensive nodal root system size (Weaver, 1926), it is difficult to study the root system in the field. Because the field situation has a high 
degree of variability, detailed physiological studies of root growth maintenance proved to be challenging. One objective of this study was to optimize a system for the study of nodal root growth under water deficit in a highly controlled and reproducible method.

Several split root studies have proven useful in elucidating the root and shoot responses to water deficit (Volkmar, 1997; Rostamza et al., 2013). Volkmar's split root system was originally designed to study the root to shoot signaling effects in wheat. In the water stress treatments, he saw a significant increase in the nodal root system, presumably due to the lateral roots on the nodal axes (Volkmar, 1997).

Rostamza's study of millet and sorghum in a divided-chamber system looked at the importance of the seminal and primary root systems in comparison to the nodal root system. In response to water stress, the sorghum and millet nodal roots became thick and shortened with no lateral roots. They also observed a steep root angle that was more dramatic in millet, and they attributed this to hydrotropism (Rostamza et al., 2013).

While both of these systems were similar to the maize divided-chamber system developed in the present study (see Chapter 2), they did not measure the water potential of the soil or of the tissue. To study the detailed physiology of the nodal roots, the level of water stress imposed on the roots needed to be highly precise and reproducible. 


\section{Taking into account plant water status}

When comparing genotypes under water stress, it is important to define the level of water stress that they are experiencing in the soil for reproducibility of the tests. But plants are uniquely suited to mitigate stress in multiple ways, either by avoiding the stress or tolerating the stress.

Plants that have limited leaf area and close their stomata readily in response to drying soil conserve the water available in the soil. Therefore, if one genotype has on average less leaf area and greater stomatal resistance, it will likely appear to have superior performance under a standard withholding of water experiment in comparison to a plant with more leaf area and less stomatal resistance, because it is not losing as much water from the soil and is experiencing less stress than the leafier genotype as a result. It is successfully avoiding the water stress by conserving its water resources.

While this response is undoubtedly key in breeding drought avoiding lines for regions that routinely experience terminal droughts, it must be differentiated from plants that are able to continue to grow while fully experiencing stress. Two plants must be at the same stress level in order to compare how drought tolerant they are in relation to each other.

\section{Objectives}

Understanding the physiology of nodal roots may reveal new strategies for crop breeding to achieve greater drought tolerance in maize. The aims of this study were to develop a highly-controlled system to characterize nodal root 
growth response to a range of low soil water potentials. The specific objectives of this study were to:

- Develop a divided-chamber system for the highly controlled and reproducible growth of maize nodal roots in response to a uniform low water potential soil.

- Characterize the growth response of maize nodal roots to a wide range of low soil water potentials (-0.1 MPa, -0.3 MPa, -0.6 MPa, -0.9 MPa, and 2.0 MPa).

- Examine for genetic diversity in the response by comparing two contrasting maize lines, FR697 and B73, which differ in the ability to maintain primary root growth under low water potentials.

- Compare the growth response of two distinct developmental nodes of roots at equivalent developmental length.

- Investigate the role of hydraulic conductivity in the root in maintaining the growth of nodal roots in drying soil. 


\section{CHAPTER 2}

Development of a new divided-chamber model system for the characterization of maize nodal root growth response to water

deficit 


\section{ABSTRACT}

This chapter discusses the importance of proper development of various factors in the divided-chamber system that affect the healthy growth and development of maize plants in the system. The present system was designed to support the growth of older maize plants while still imposing the same homogenous low water potential and supporting steady-state growth of the nodal roots. In order to best study this transition of lab to the field, the number of artifacts due to artificial growing conditions was reduced as much as possible. Complications with soil aeration in the inner chamber led to the development of an aeration system for the seminal and primary roots. Experiments with additional stress conditions may be possible in the future. 


\section{INTRODUCTION}

A model system developed by Sharp et al. was instrumental in characterizing the primary root growth response of maize seedlings to low water potentials (Sharp et al., 1988; Yamaguchi and Sharp, 2010). The system was particularly useful for the characterization of growth because of the highly controlled and reproducible environment in which the seedlings were grown.

In the primary root model system, germinated seedlings of uniform developmental stage are transplanted into vermiculite of a uniform and predetermined water potential. Uniformity of media water potential ensures that as the root elongates into new volumes of soil, it will encounter the same water potential, unlike the gradient of soil water potentials that is produced through withholding water.

Seedlings are non-transpiring at this stage of development. Transpiration translocates water from the media to the atmosphere, changing the water potential and stress level of the media. To minimize transpiration as shoots begin to develop, seedlings are grown in the absence of photosynthetically active radiation. Although the young shoot may have few fully developed stomata, the seedlings are protected from light which may trigger the opening of stomata. Instead, a dim green light is used while handling the seedlings. To further preserve the media's defined water potential throughout the experiment, the plants are grown in near-saturation humidity. Keeping the system in nearsaturation humidity ensures that little to no water is lost to the environment (Sharp et al., 1988). 
Another benefit of the system is the ability to observe root growth patterns throughout an experiment. Seedlings are transplanted and grown against the transparent face of a Plexiglas box, where root growth is marked. Calculation of root growth rate and the determination of steady-state root growth are made possible through root marking data.

Plants are grown at a constant temperature to facilitate a steady-state growth rate, which is highly reproducible and enables the comparison of results between experiments. The constant temperature also prevents condensation from forming on the walls of the Plexiglas box, which would alter the water potential of the growth media against the face of the box.

However, the vermiculite culture system is limited to the study of seedling physiology, because the system is non-transpiring and excludes light, both of which are necessary for the healthy development of the shoot. In addition, the low water potential media commonly used in the system inhibits shoot growth. At tissue water potentials below $-0.3 \mathrm{MPa}$, maize shoot growth becomes inhibited, and shoot growth is completely inhibited below -0.8 MPa (Sharp et al., 1988). Inhibition of shoot growth would inhibit the growth of nodal roots as development of a healthy shoot is necessary for the formation of nodal roots (Hoppe et al., 1986). If these complications with shoot growth were not addressed, this system would be unsuitable for studying nodal root growth under severe water deficit conditions. Therefore, a new model system was developed to study the growth of nodal roots, which included a light source and a water source for healthy shoot development. 
The design of the new model system was developed from combining the vermiculite culture system previously described (Sharp et al., 1988) with a split root system, previously developed to study root to shoot signaling in wheat (Volkmar, 1997). Concurrent to the development of the present system, another split root system was developed to study the growth of sorghum and millet roots in response to water stress, imposed by withholding water from nodal or seminal roots (Rostamza et al., 2013).

Similar to the primary root model system, the nodal root model system was designed to impose a pre-determined and uniform low water potential on the nodal root system. This was achieved by allowing the nodal roots to emerge into a chamber that was filled with the user-defined uniform water potential media. As nodal roots elongate into new volumes of soil, they experience a defined, steadystate, and reproducible soil water potential that allows precise characterization of nodal root growth responses to a wide range of precisely-imposed soil water deficit conditions.

In order to maintain shoot growth and enable transpiration while imposing stress on the nodal roots, a second inner chamber was developed to supply water to the shoot (Fig. 1A). This inner chamber was designed to contain the seminal and primary root systems, and it would remain well-watered throughout the experiment. The rate of transpiration is tightly regulated by the plant through hydraulic and chemical signals as previously reviewed (Comstock, 2001). Some water loss through transpiration is beneficial to the plant: The energy lost through evaporation cools leaves during heat stress, and the movement of water in the 
xylem is used as a long-distance pathway for water, nutrients, and natural products in the plant (Taiz and Zeiger, 2010). Complete inhibition of transpiration may negatively impact the growth of the shoot and negatively affect the rate of photosynthesis (Lawlor and Cornic, 2002), creating an artifact of the system.

Maintaining a high shoot, seminal root, and primary root water potential was also necessary, so that all stress imposition was initially perceived by the nodal root system. Provision of water through the inner chamber minimized stress imposition on other organs of the plant by supplying water directly to the seminal and primary root systems and indirectly to the shoot, which is hydraulically connected to the seminal and primary root systems through the mesocotyl (Hoppe et al., 1986).

The media used in the divided-chamber system needed to provide adequate structure and texture to allow for healthy cation exchange, air movement, and water holding capacity. Wet vermiculite from the vermiculite culture system loses its texture and compresses over time, creating a variable and detrimental environment for roots. To determine if vermiculite would sustain the plant over time, a peat-based media and vermiculite were compared. Due to the enclosed nature of the inner chamber, another concern was to address whether aeration of the inner chamber was necessary for the growth of healthy plants in the divided-chamber system.

Two contrasting maize genotypes were selected based on a previous study of maize primary root growth maintenance at low water potentials (Leach et al., 2011) to characterize nodal root growth responses to low water potential soil 
in the divided-chamber system. FR697 was selected for its superior ability to maintain primary root growth under water deficit while B73 showed higher sensitivity to low soil water potentials (Leach et al., 2011). In addition to the advantage of comparing the differential regulation of physiological processes between the lines, B73 also represents a large genetic and genomic resource that could be leveraged in future studies with mutants and transgenic lines (Schnable et al., 2009). The genome of B73 has been sequenced, and it serves as the common parent among the Nested Association Mapping (NAM) Recombinant Inbred Lines (RILs) (McMullen et al., 2009).

The objective of this study was to determine the optimal growing conditions for the maize inbred lines FR697 and B73 in the new divided-chamber model system, and to optimize the aeration design of this system for the study of nodal root growth response to water deficit conditions.

\section{MATERIALS AND METHODS \\ Water potential measurements}

Soil water potentials were measured using isopiestic thermocouple pyschrometry (Boyer and Knipling, 1965).

\section{Growth media and preparation}

ProMix BX (Premier Tech Horticulture, Rivière-du-Loup, Québec, Canada) was used as the growth media (also referred to here after as soil, despite its lack of structure normally attributed to soil) following germination. 
ProMix is $75-85 \%$ Canadian sphagnum peat moss, with additions of perlite, vermiculite, dolomitic and calcitic limestone, and a wetting agent. The soil was sieved before nutrient solution was added to increase the homogeneity of the soil mixture.

The following custom macro-nutrient solution (DG Blevins, University of Missouri, personal communication) was added to the soil: $0.5 \mathrm{mM} \mathrm{KH}_{2} \mathrm{PO}_{4}, 1.25$ $\mathrm{mM} \mathrm{MgSO}_{4}-7 \mathrm{H}_{2} \mathrm{O}, 2.5 \mathrm{mM} \mathrm{Ca}\left(\mathrm{NO}_{3}\right)_{2}-4 \mathrm{H}_{2} \mathrm{O}, 2.5 \mathrm{mM} \mathrm{KCl}$. The following maize micro-nutrient solution was added to the soil: $2.3 \mu \mathrm{M} \mathrm{H} \mathrm{H}_{3} \mathrm{BO}_{3}, 0.9 \mu \mathrm{M} \mathrm{MnSO}_{4}-\mathrm{H}_{2} \mathrm{O}$, $0.6 \mu \mathrm{M} \mathrm{ZnSO}_{4}-7 \mathrm{H}_{2} \mathrm{O}, 0.1 \mu \mathrm{M} \mathrm{NaMoO} 4-2 \mathrm{H}_{2} \mathrm{O}, 0.11 \mu \mathrm{M} \mathrm{NiCl} 2-6 \mathrm{H}_{2} \mathrm{O}, 0.15 \mu \mathrm{M}$ $\mathrm{CuSO}_{4}-5 \mathrm{H}_{2} \mathrm{O}$. Iron chelate (Sprint 138, Becker Underwood) was added at the rate of $2.5 \mathrm{~g} \cdot \mathrm{L}^{-1}$ nutrient solution. Nutrient solution was added to the drained capacity of the soil, approximately $1 \mathrm{~L}$ nutrient solution for $2 \mathrm{~L}$ sieved soil.

Soil wetted with nutrient solution was dried in a $55^{\circ} \mathrm{C}$ forced-air oven for 48 hours or until a constant weight. The custom nutrient solution was selected to prevent ammonia fumes while drying. Drying down the soil after adding nutrients also ensures the nutrients would bind to the cation exchange sites in the soil and be displaced evenly throughout the soil. Soil was then rehydrated to well-watered drained capacity or to a uniform soil water potential with deionized water.

It was considered that a slow-release fertilizer such as Osmocote beads would not be suitable for these experiments focused on precise and highly reproducible soil water potentials, due to the pockets of low water potential that would occur in the soil as the osmotic potential drops suddenly near a dissolving Osmocote bead. If a root encountered such a pocket of low water potential, it 
would not only add heterogeneity of water availability but also heterogeneity of nutrients in the soil, which has been shown to affect root growth (Hodge, 2004).

Soil from the experiment testing the effects of soil drying on root growth was dried at $75^{\circ} \mathrm{C}$. The oven temperature was reduced to $55^{\circ} \mathrm{C}$ after it was discovered that temperatures above $60^{\circ} \mathrm{C}$ may affect the carbon and nitrogen content of the soil (Harmon and Lajtha, 1999).

\section{Growth of plant materials and imposition of water stress}

Seed of maize inbred lines B73 and FR697 were surface sterilized with $5 \%$ bleach solution and imbibed in $1 \mathrm{mM} \mathrm{CaSO}_{4}$. Imbibition is proposed to release germination inhibitors from the seed and synchronize germination rate, so that the seed begins germination at the same relative time. After imbibition, seeds were placed in vermiculite and wetted to container capacity with $1 \mathrm{mM}$ $\mathrm{CaSO}_{4}$ (Spollen et al., 2000). Seeds were allowed to germinate in the dark at 29 $\pm 1^{\circ} \mathrm{C}$ and near-saturation humidity.

The growth of the plants in the divided-chamber root system was divided into two phases. Seedlings were transplanted in the dark at $29 \pm 1^{\circ} \mathrm{C}$ and nearsaturation humidity into Phase I by orienting the shoot of a seedling through the top of a $5 \times 4 \mathrm{~cm}$ plastic cone. The cone was filled with well-watered soil and attached to a polyvinyl chloride pipe ( $5 \mathrm{~cm}$ internal diameter $\times 75 \mathrm{~cm}$ long), which was filled with well-watered soil. The bottom of the inner chamber was open and freely draining. The primary root was $2-6 \mathrm{~cm}$ long at the time of transplant. 
A temporary sleeve of well-watered soil was wrapped around the top of the cone to ensure the coleoptile would not be damaged by hardened top soil during emergence. The height of the sleeve was uniform, so that the tip of the coleoptile would emerge from the soil surface approximately $5 \mathrm{~cm}$ above the placement of the seed.

After transplant, the Phase I plants were placed in a Conviron model PGW36 growth chamber. The chamber was programmed to maintain a constant $29^{\circ} \mathrm{C}$ soil temperature during the night and day, the optimal temperature for maize seedling germination (Miedema et al., 1987). To maintain a constant soil temperature, the air temperature was $26^{\circ} \mathrm{C}$ during the light cycle and $29^{\circ} \mathrm{C}$ during the dark cycle. Soil temperature was monitored with soil temperature probes (Hobo Pro V2 External) in the outer chamber, which was most sensitive to fluctuations in soil temperature.

The light cycle was 14 hours long with a light intensity of $600+/-50$ $\mu \mathrm{mol} \cdot \mathrm{m}^{-2} \cdot \mathrm{sec}^{-1}$ provided by a mixture of fluorescent and incandescent bulbs at the top of the leaf canopy. Relative humidity was $85 \%$, designed to decrease the rate of transpiration and help maintain the water potential of the soil in the outer chamber.

Approximately two days after transplant, plants were transferred to Phase II through excavation of the young shoot from the sleeve of well-watered soil (Volkmar, 1997). Plants were excavated in the dark at $29 \pm 1^{\circ} \mathrm{C}$ and nearsaturation humidity. During excavation, the soil and sleeve surrounding the shoot were carefully removed, and the inner chamber containing the seminal and 
primary root system was rolled inside of the outer chamber, composed of a large transparency film sheet containing the uniform water potential soil. The outer chamber had an internal diameter of $11 \mathrm{~cm}$ and was either $45 \mathrm{~cm}$ or $60 \mathrm{~cm}$ long to accommodate nodal root lengths obtained in 7- and 10-day duration experiments (see Chapter 3).

Plants were transitioned to Phase II when the first true leaf began to expand (Fig. 2). If the plants were transitioned to the outer chamber before the first true leaf began to expand, the mesocotyl continued to elongate, and the crown set above the soil surface, exposing the nodal roots to light and the low water potential air. If plants were excavated after this developmental stage, nodal root initials were present and had emerged into the well-watered soil of the temporary sleeve. Nodal roots that were present at time of excavation became damaged and did not adapt to the dry soil in the outer chamber. If plants were not excavated at the appropriate developmental stage, they were omitted from the study.

The outer chamber was wrapped in aluminum foil, including the top of the soil to limit evaporation and heating of the surface soil. The assembled system with the growing plant was placed in the growth chamber. Plants were weighed after excavation and watered back to weight every second day with de-ionized water through a straw inserted into the inner chamber. Watering back by weight is suggested to reproduce a consistent water content and prevent over-watering of the seminal and primary roots systems (Passioura, 2006). 
The transparency of the outer chamber was sturdy, clear, and waterproof, so it made a suitable face for marking root growth. Nodal root growth was marked daily during the night cycle, using a dim green light that did not interfere with root growth (Saab et al., 1990; Saab et al., 1992). Growth rates were calculated from the root length increase marked along the side of the outer chamber divided by the time interval between markings.

Plants were grown in Phase II for seven or ten days before harvest. To ensure the second node of roots from both genotypes in the well-watered condition were a similar length at the extended harvest, B73 plants were harvested approximately 12 hours earlier than FR697 plants, due to a genotypic difference in the timing of second node root initiation.

\section{Aeration}

Although the top of the chamber was closed, the bottom of the inner chamber was open and freely draining, which allowed air circulation through the bottom of the chamber and helped prevent over-saturation of the media. To increase bulk diffusion of air flow through the top and middle of the inner chamber, two pipettes were inserted through the entire length of the inner chamber. The pipettes were perforated to increase the surface area of air diffusion into the media. Air was pumped into the inner chambers at an approximate rate of $10 \mathrm{~L} \cdot \mathrm{min}^{-1} \mathrm{using}$ fish tank aerators. Unless otherwise noted, air was humidified by bubbling through water before entering the pipettes in the inner chamber. 
To test whether the entry and exit sites of air movement affected the plant, tests were performed that looked at top-fed and bottom-fed aeration systems. Top-fed aeration was pumped through the top of the cone, which featured two pipettes running the length of the inner chamber with air holes along the pipettes to increase the surface area of air diffusion with an open lid at the bottom of the tube for air to escape. Bottom-fed aeration was pumped through one entry point at the bottom of the tube, which had a sealed bottom lid with two exit straws at the top of the cone for air to escape. To test whether the aeration of the outer chamber affected the plant, tests were performed using three similarly perforated pipettes to introduce air into the outer chamber.

\section{Leaf tip burn rating}

A qualitative score of leaf tip burn severity was assigned to leaves 4 and 5, which exhibited leaf tip burn symptoms. Leaf tip burn scale: Yellow discoloration of the leaf tip (1), Severe discoloration of the leaf tip (2), Discoloration and deformation of leaf tip (3), Severe deformation of the leaf tip (4).

\section{Statistical analysis}

For analysis of root growth in different soil drying treatments, a student's ttest was performed to determine statistical significance. For analysis of leaf tip 
burn ratings, a nonparametric t-test was performed to determine statistical significance through the Kruskal-Wallis test (Sokal and Rohlf, 1995).

\section{RESULTS AND DISCUSSION \\ Peat-based media supports nodal root growth}

The growth rate of nodal roots in well-watered vermiculite decelerated over time (Fig. 3). Conversely, the nodal root growth rate of those plants in wellwatered ProMix accelerated over time and was approaching steady-state. Additionally, nodal roots growing in peat-based media grew at a faster rate than the vermiculite treatment (Fig. 3).

Because nodal root growth was inhibited in vermiculite relative to the peatbased ProMix, it was not considered suitable for supporting the growth of a healthy maize plant in the nodal root model system. Instead, it was decided that the peat-based media would be used, which successfully supported the growth of maize.

\section{Nutrient type}

An experiment was performed to evaluate the effects of various types of soil drying to test if rehydrating the soil after drying it in the oven would affect the growth of the roots. There was no significant effect on seminal root system length (including root axes and lateral roots) or nodal root system length when the soil was dried in the oven or on the lab bench in the air relative to soil that had not 
Figure 1. Design of a divided-chamber model system to study the response of maize nodal root elongation to variation in soil water potential independently of the primary and seminal roots (A). Dimensions of compartments are not to scale. Aeration and watering tubes for inner container are not shown. Representative B73 (B) and FR697 (C) maize plants grown for 7 days in the divided-chamber system and treated with well-watered soil in both chambers. Yellow bars indicate the division between the chambers. 

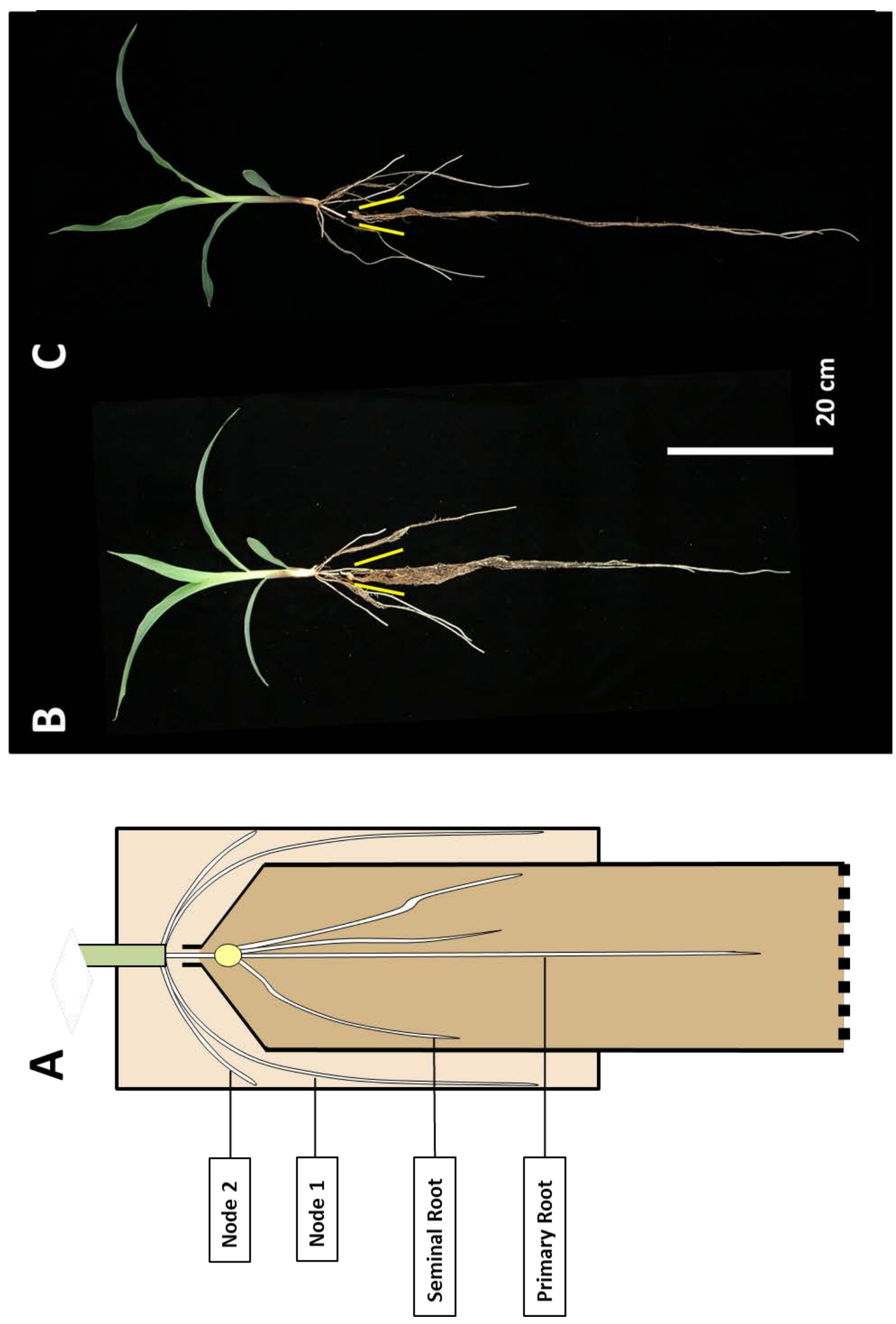
Figure 2. B73 (A) and FR697 (B) shoots at the developmental stage for excavation from Phase I to Phase II. During excavation, the inner chamber is placed inside the outer chamber to form the completed divided-chamber system.

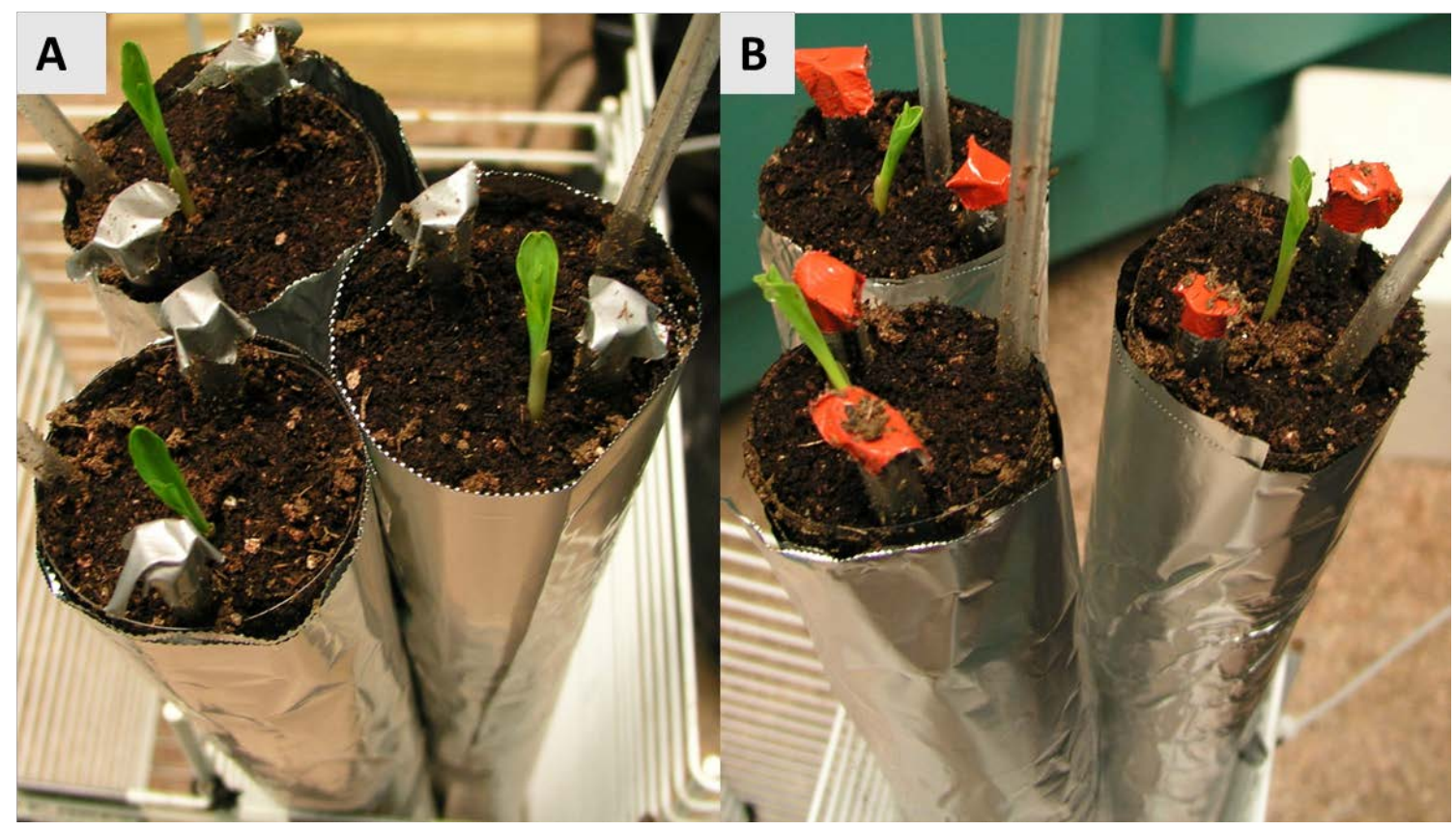


Figure 3. Root elongation rate of B73 Node 1 roots in the divided-chamber system, treated with well-watered vermiculite or peat-based ProMix in the outer chamber. Data are means \pm SE ( $n>3$ roots).

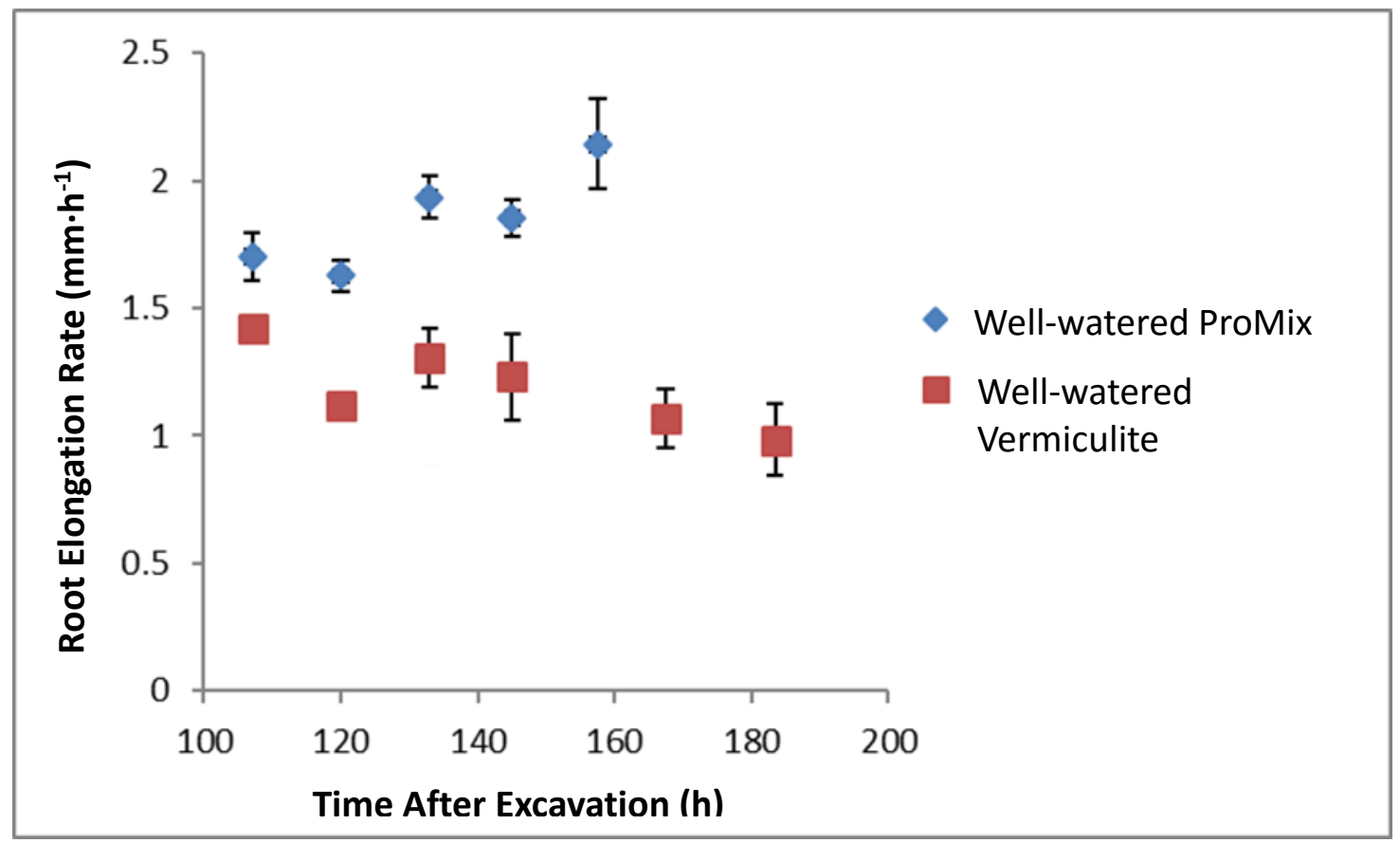


been dried (Fig. 4). Because drying soil in the oven did not affect root growth, and speeds up the drying process, a soil drying process was adopted.

\section{Aeration optimization}

Because the inner chamber was well-watered and sealed at the top cone, air circulation in the inner chamber was low and the issue of oxygen availability needed to be addressed. Possibly as a complication with hypoxia, a severe leaf tip burn developed, causing a yellow discoloration that progressed into leaf deformation (Fig. 5B). Because the youngest leaves were most affected by the tip burn, they deformed around the whorl of young leaves, encumbering new leaves from emerging.

Adequate level of aeration was measured by the level of severity of leaf tip burn and the root to shoot ratio, which is a sensitive indicator of hypoxia (Horchani and Aschi-Smiti, 2010). Because the hypoxic condition occurred most dramatically in the well-watered condition (data not shown), low water potential soil treatments in the outer chamber were not included in the initial optimization of aeration in the divided-chamber system.

Aeration of the inner chamber with ambient or humidified air alleviated tip burn (Figs. 5A, 6). Aeration with ambient air also significantly increased the root, shoot, and total plant dry weight in comparison to plants that were not aerated (Fig. 7A). Because the root to shoot ratio was higher in plants that were 
Figure 4. Total seminal root system length $(A)$ and total nodal root system length (B) of B73 plants grown in $25 \mathrm{~cm} \times 10 \mathrm{~cm}$ pots for 7 days with soil that had been air dried, oven dried at $75^{\circ} \mathrm{C}$, or not dried before planting. There was no significant difference in length between the various soil drying methods (t-test). Data are means \pm SE $(n=5-6$ plants $)$.

A

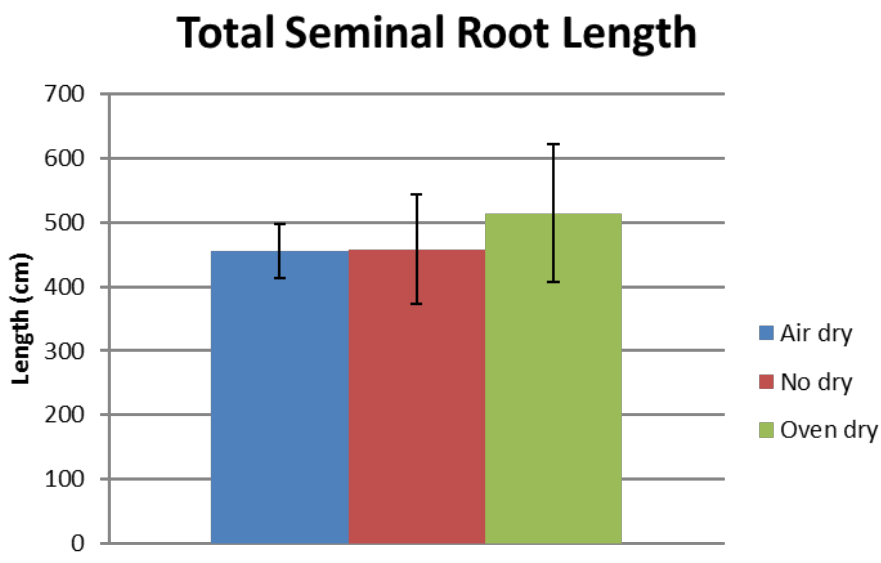

B

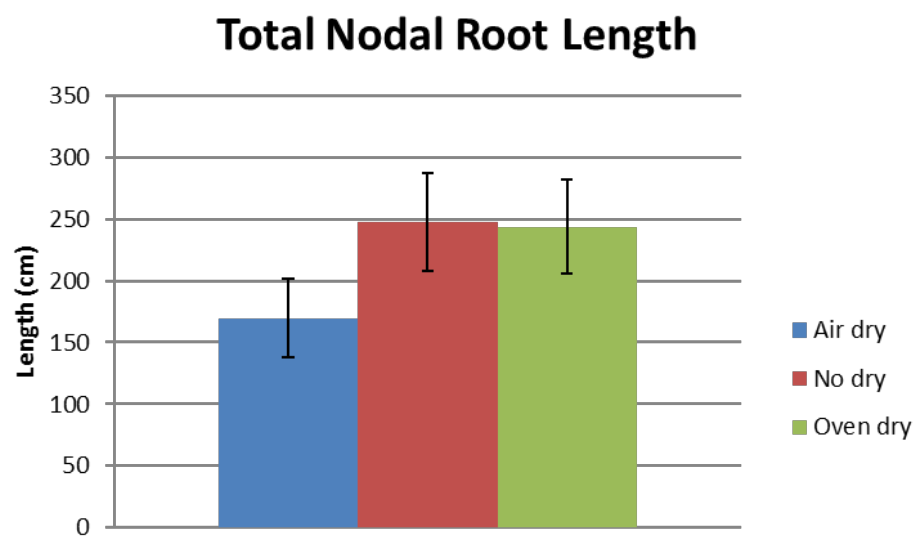


Figure 5. B73 shoots that were aerated in the inner chamber with humidified air (A) or not aerated (B). Shoots of plants that were not aerated showed leaf tip discoloration and deformation, encumbering the emergence of younger leaves. Plants were harvested after 7 days in the divided-chamber system and treated with well-watered soil in the outer and inner chambers.

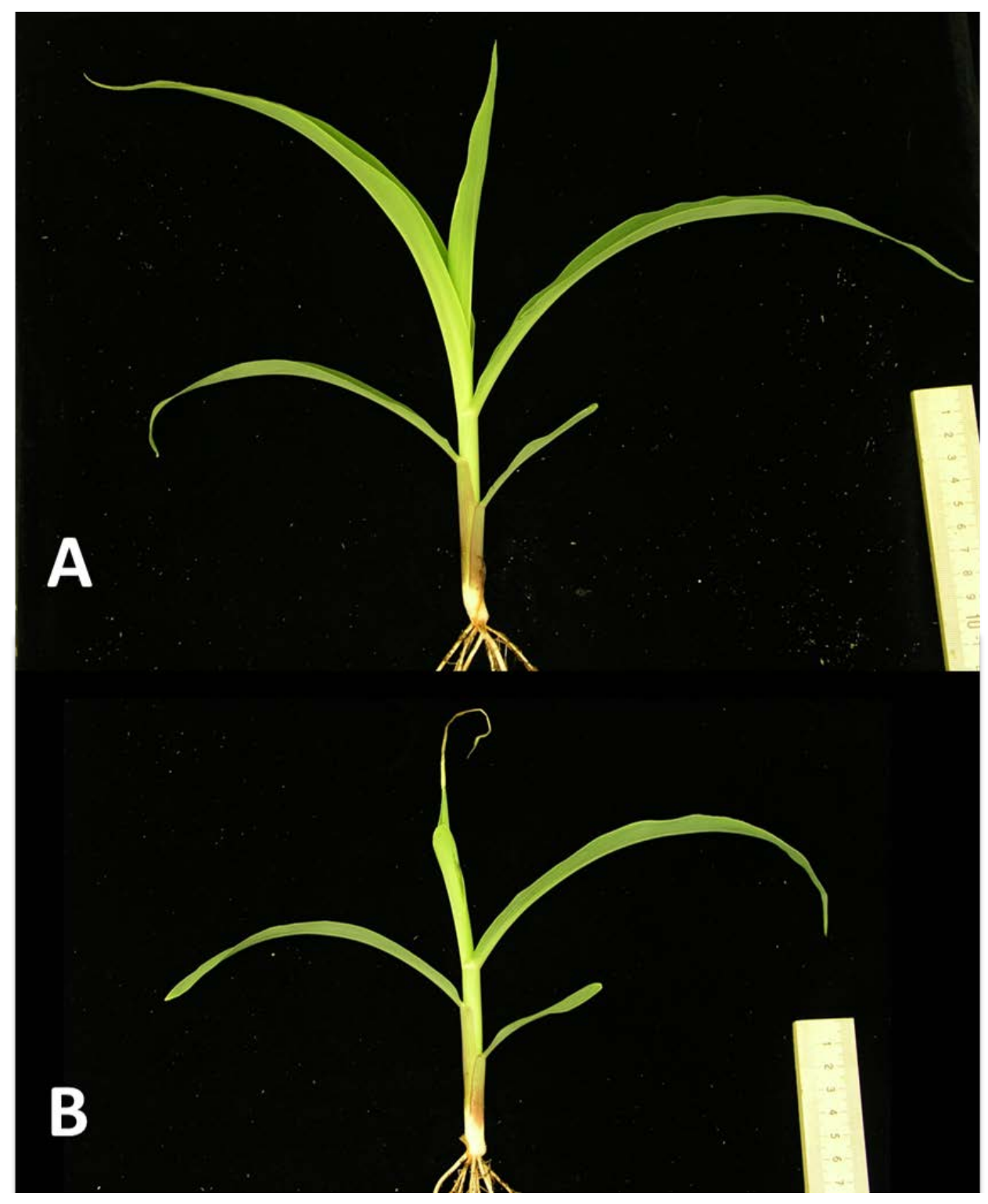


Figure 6. Leaf tip burn rating of B73 plants grown in the divided-chamber system with various techniques that were tested for best method of inner chamber aeration. Leaf tip burn was highest on leaf 4 . Aeration in the inner chamber with humidified or ambient air both significantly reduced the rating of tip burn on the leaves. Plants were harvested after 7 days in the divided-chamber system. Leaf tip burn scale: $1=$ Yellow discoloration of the leaf tip, $2=$ Severe discoloration of the leaf tip, 3=Discoloration and deformation of leaf tip, 4=Severe deformation of the leaf tip. Data are means \pm SE $(n=4-5$ plants). Means with different letters are significantly different, $\mathrm{P}<0.05$.

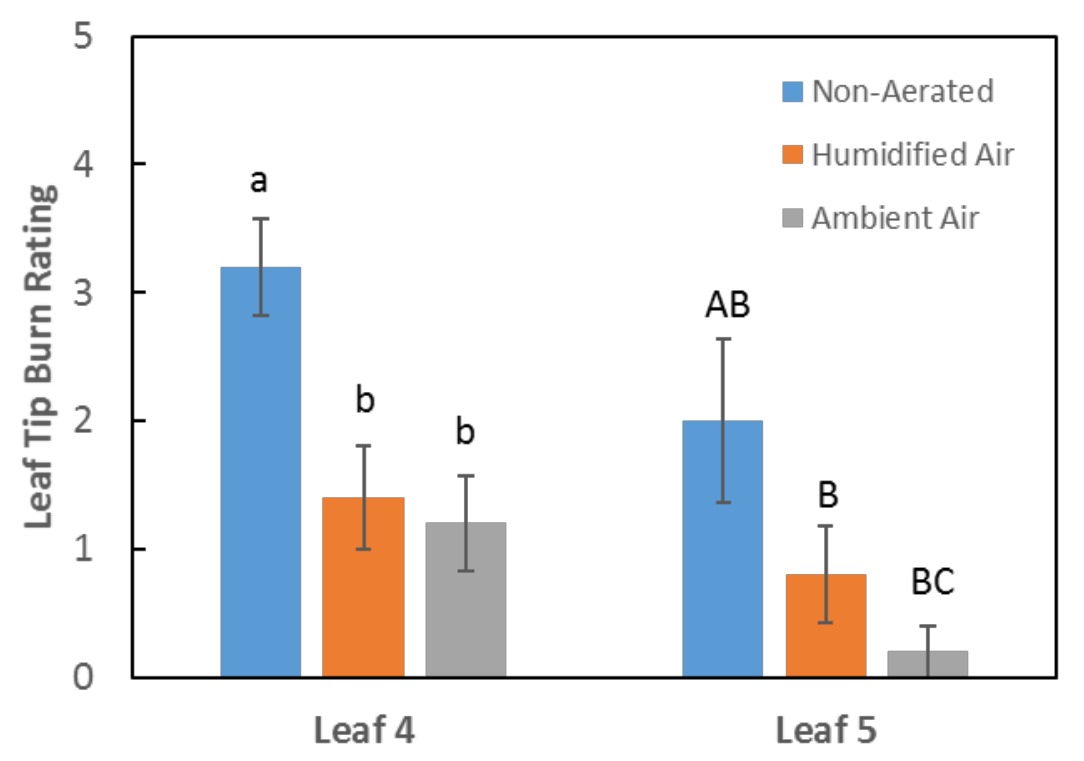


Figure 7. Dry weight of root and shoot tissue (A) and root to shoot dry weight ratio (B) of B73 plants grown in the divided-chamber system with various techniques that were tested for best method of inner chamber aeration. Aeration in the inner chamber with ambient air significantly increased the root, shoot, and total plant dry weight, but the root to shoot ratio significantly increased when plants were aerated with humidified air. Plants were harvested after 7 days in the divided-chamber system. Data are means \pm SE $(n=4-5$ plants). Asterisks denote significant differences between the non-aerated and aerated plants (t-test: $\left.{ }^{\star} \mathrm{P}<0.05\right)$.

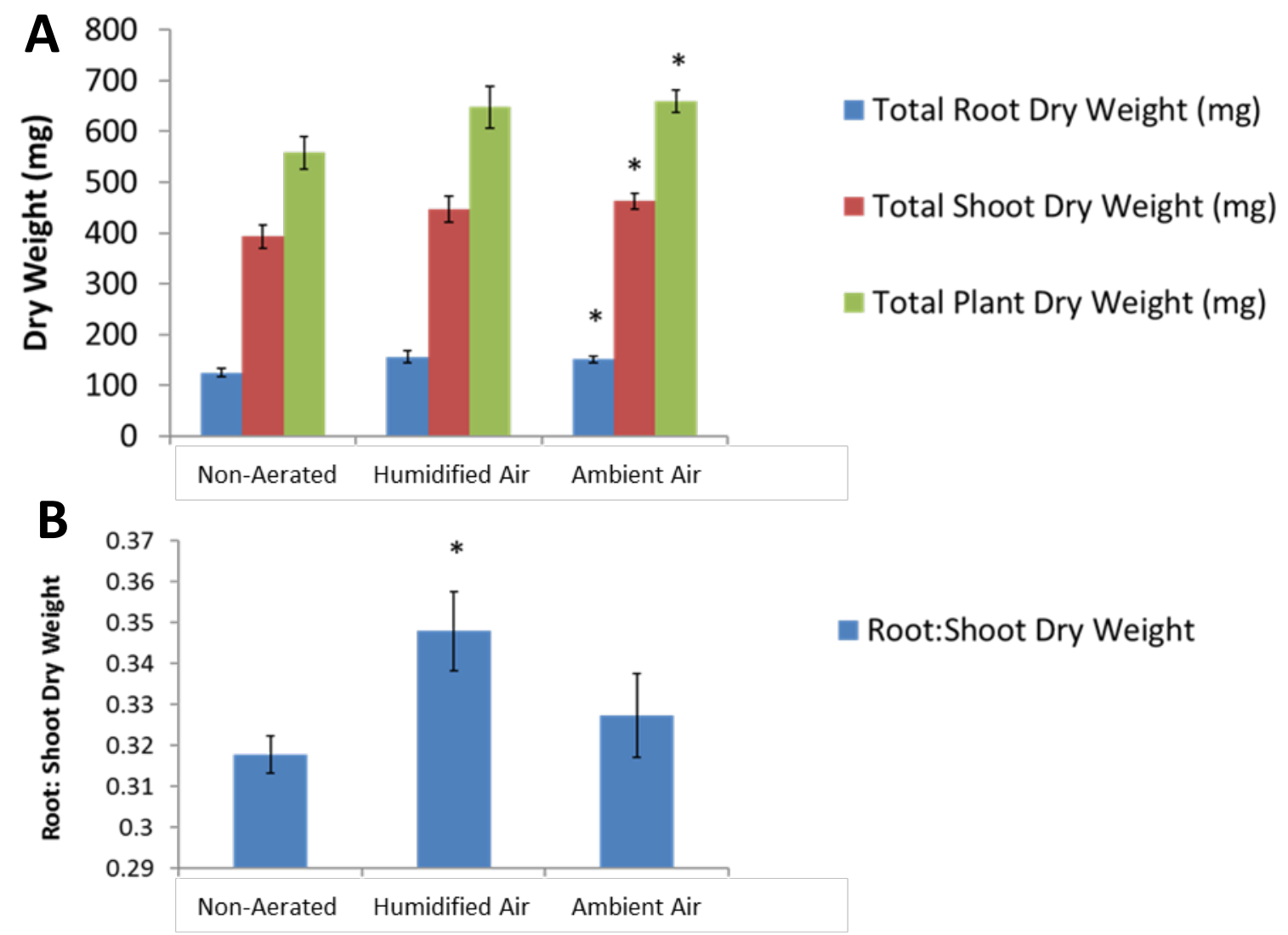


aerated with humidified air (Fig. 7B), humidified air was selected for the system to prevent any possible disturbance of the roots.

Aeration through the top or bottom of the inner chamber significantly reduced the severity of tip burn on the leaves (Figs. 8, 9, and 11). Top-fed aeration also significantly increased the root dry weight of plants in comparison to non-aerated plants (Fig. 10A). Because the root to shoot ratio increased significantly in top-fed aerated plants (Fig. 10B), this entry point for aeration was adopted for the final divided-chamber aeration system.

Outer chamber aeration did not have a significant effect on leaf tip burn rating (Figs. 12 and 13). Additionally, outer chamber aeration would change the water status of soil in the outer chamber. Therefore, outer chamber aeration was not included in the final divided-chamber aeration system.

To ensure that enough air was circulating through the inner chamber, the rate of aeration was doubled. Both rates of aeration had similar effects on significantly decreasing leaf tip burn (Fig. 14). The lesser rate was adopted in the final divided-chamber aeration system to minimize any disturbance aeration may be causing to the roots.

\section{Steady-state nodal root growth rate}

B73 and FR697 plants were grown in the divded-chamber system (Fig. 1) with well-watered or low water potential soil (-0.3 MPa, $-0.9 \mathrm{MPa})$ in the outer chamber. Growth rate data from the extended experiments indicate nodal roots 
Figure 8. Diagram of the divided-chamber system and various techniques that were tested for best method of inner chamber aeration. Top-fed air (A) featured two pipettes running the length of the inner chamber with air holes along the pipettes to increase the surface area of air diffusion with an open lid at the bottom of the tube for air to escape. Bottom-fed air (B) featured one point of air entry at the bottom of the tube, which has a sealed bottom lid with two exit straws at the top of the cone for air to escape. Non-aerated plants (C) featured the same two pipettes running the length of the inner chamber with air holes along the pipettes and an open lid at the bottom of the tube, but air was not fed into the pipettes.

A

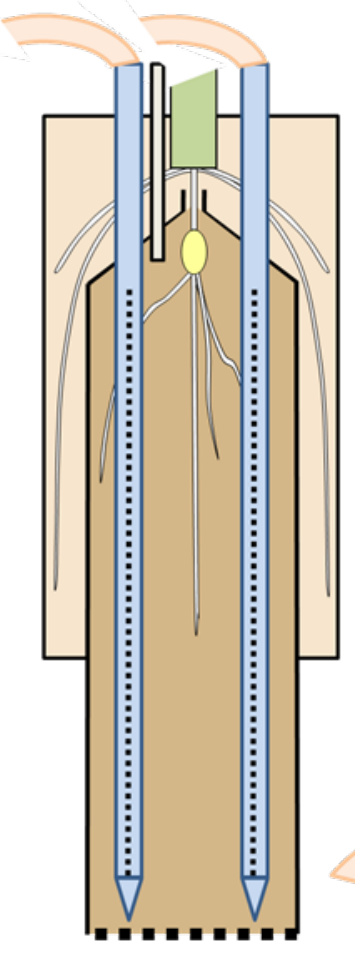

B

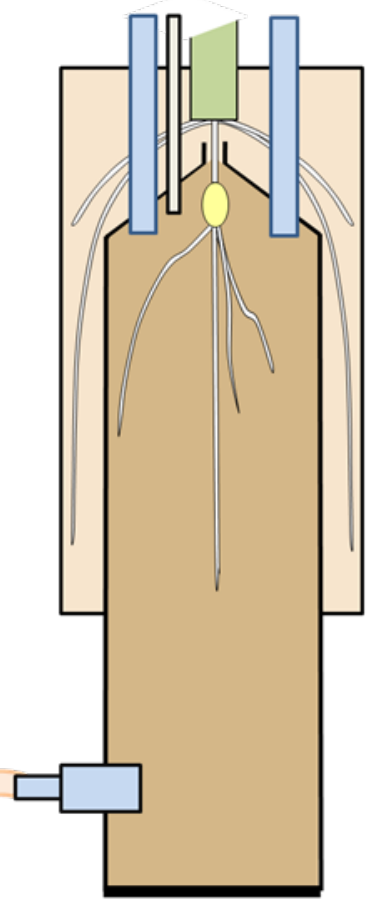

C

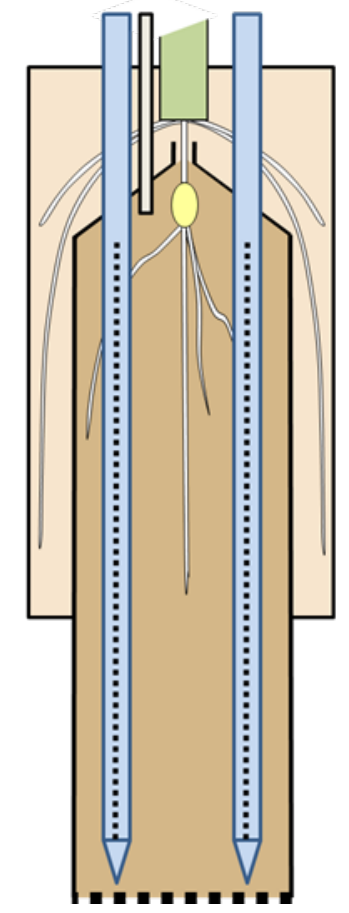


Figure 9. Leaf tip burn rating of B73 plants grown in the divided-chamber system with various techniques that were tested for best method of inner chamber aeration. Leaf tip burn was highest on the most immature leaf, leaf 5. Aeration through the top and bottom both significantly reduced the rating of tip burn on the leaves. Plants were harvested after 7 days in the divided-chamber system. Leaf tip burn scale: $1=$ Yellow discoloration of leaf tip, 2=Discoloration and deformation of leaf tip. Data are means $\pm S E(n=4$ plants). Means with different letters are significantly different, $\mathrm{P}<0.05$.

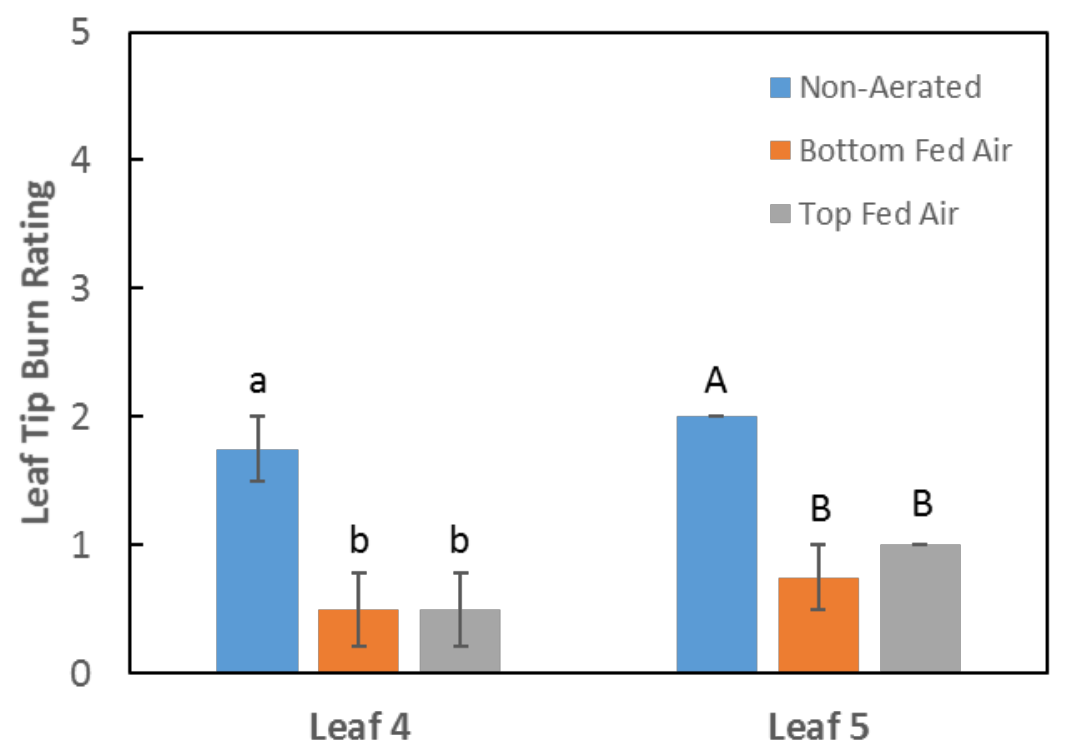


Figure 10. Dry weight of root and shoot tissue $(A)$ and root to shoot dry weight ratio (B) of B73 plants grown in the divided-chamber system with various techniques that were tested for best method of inner chamber aeration. Aeration through the bottom significantly increased the total root dry weight, but the root to shoot ratio significantly increased in top-fed aeration. Plants were harvested after 7 days in the divided-chamber system. Data are means \pm SE $(n=4$ plants $)$. Asterisks denote significant differences between the non-aerated and aerated plants (t-test: $\left.{ }^{*} \mathrm{P}<0.05\right)$.

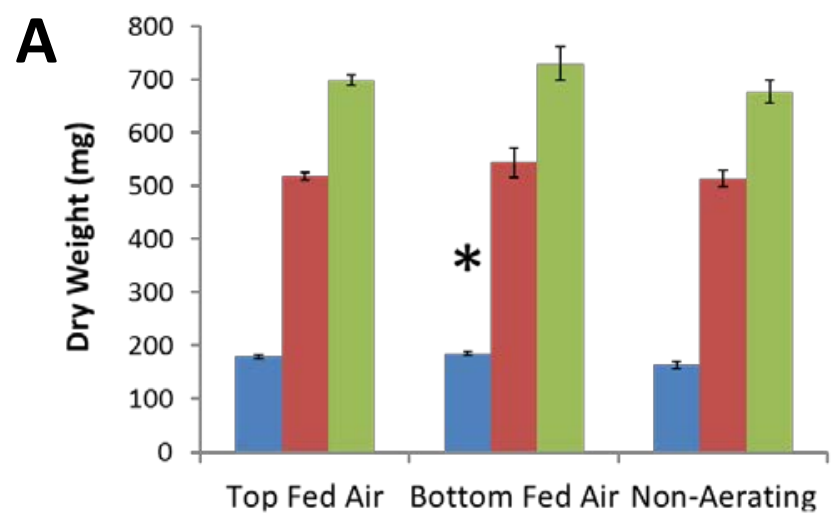

- Total Root Dry Weight (mg) - Total Shoot Dry Weight (mg)

$\square$ Total Plant Dry Weight (mg)

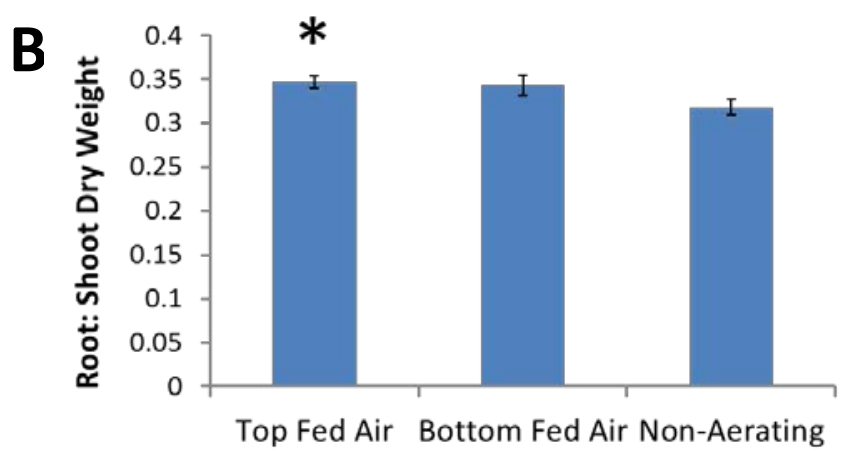

Root:Shoot Dry Weight 
Figure 11. Photographs of B73 shoots of plants that were aerated in the inner chamber through the top of the cone $(A)$, through the bottom of the tube (B), or not aerated (C). Shoots of plants were not aerated showed leaf tip discoloration and deformation, encumbering the emergence of younger leaves. Plants were harvested after 7 days in the divided-chamber system and treated with wellwatered soil in the outer chamber. 


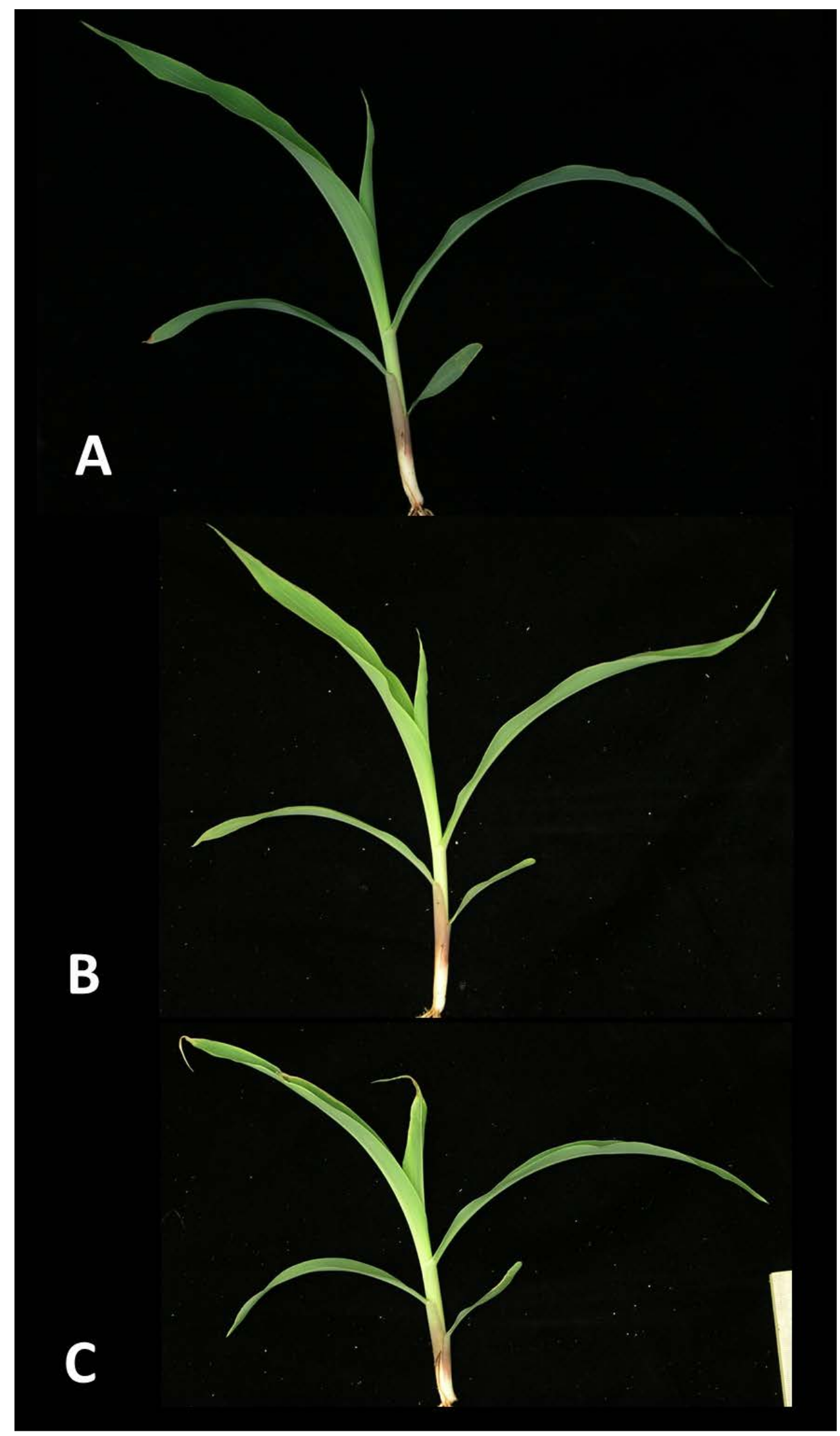


Figure 12. Diagram of the divided-chamber system and various techniques that were tested for best method of aeration. Inner chamber aeration (A) featured two pipettes running the length of the inner chamber with air holes along the pipettes to increase the surface area of air diffusion with an open lid at the bottom of the tube for air to escape. Outer chamber aeration (B) featured the same inner chamber aeration with an additional set of pipettes aerating the outer chamber.

A

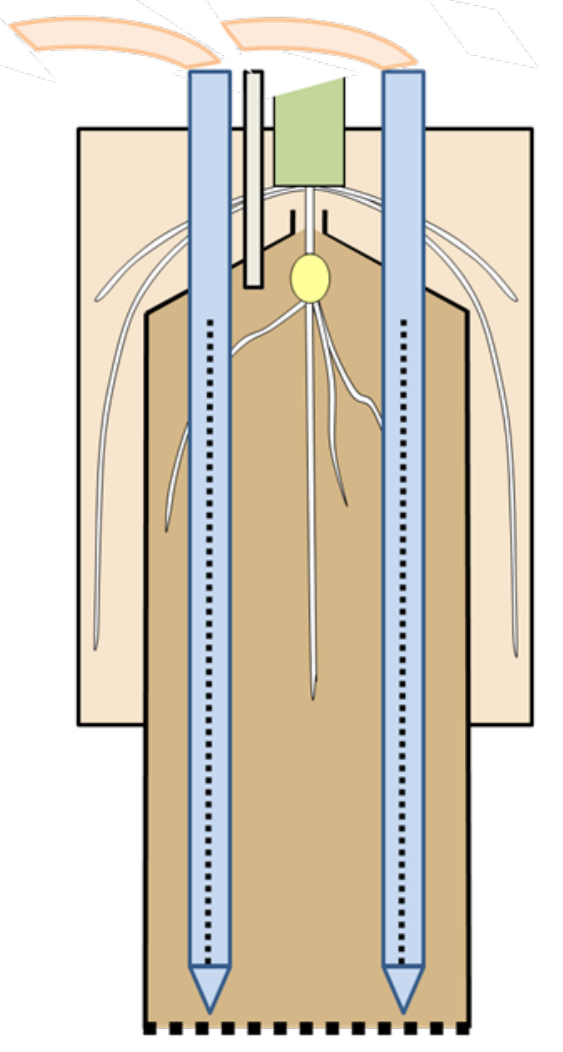

B

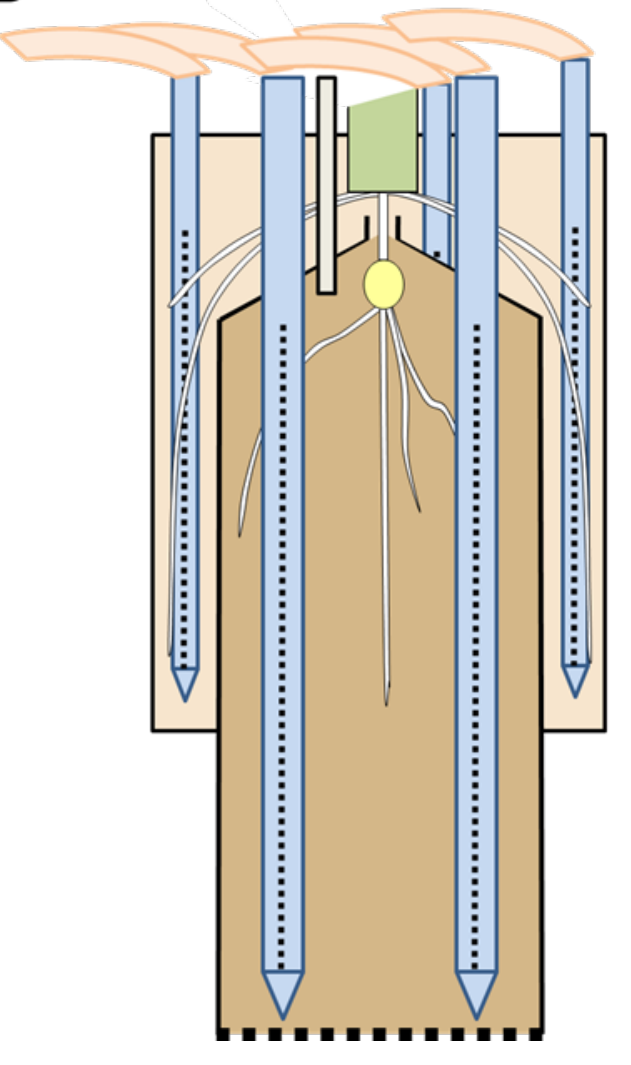


Figure 13. Leaf tip burn rating of B73 plants grown in the divided-chamber system with various techniques that were tested for best method of aeration. Leaf tip burn was highest on leaf 4 . Outer chamber aeration did not have a significant effect on leaf tip burn rating (t-test). Plants were harvested after 7 days in the divided-chamber system. Leaf tip burn scale: $1=$ Yellow discoloration of the leaf tip, 2=Severe discoloration of the leaf tip, 3=Discoloration and deformation of leaf tip, $4=$ Severe deformation of the leaf tip. Data are means \pm SE $(n=4$ plants). Means with the same letter are not significantly different, $p>0.05$.

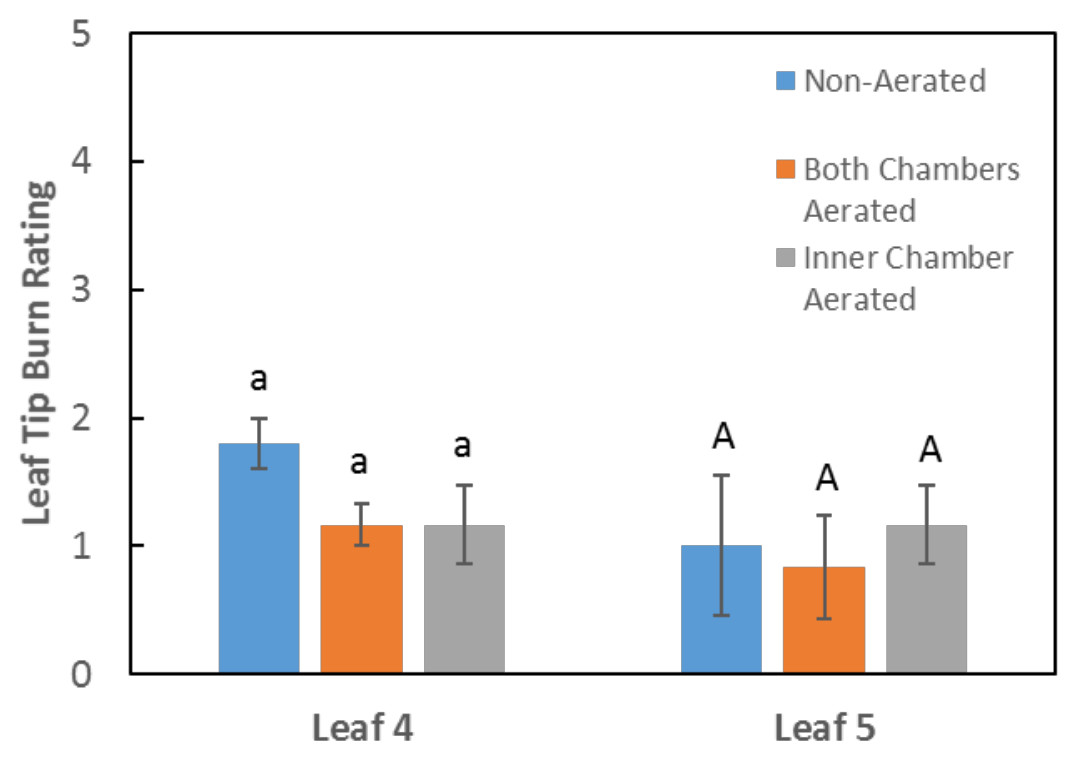


Figure 14. Leaf tip burn rating of B73 plants grown in the divided-chamber system with various techniques that were tested for best method of inner chamber aeration. Leaf tip burn was highest on leaf 6 . Both $1 x$ and $2 x$ rates of aeration had similar effects on significantly decreasing leaf tip burn. Plants were harvested after 7 days in the divided-chamber system. Leaf tip burn scale: $1=$ Yellow discoloration of the leaf tip, 2=Severe discoloration of the leaf tip, 3=Discoloration and deformation of leaf tip, 4=Severe deformation of the leaf tip. Data are means \pm SE $(n=3-5$ plants $)$. Means with different letters are significantly different, $\mathrm{P}<0.05$.

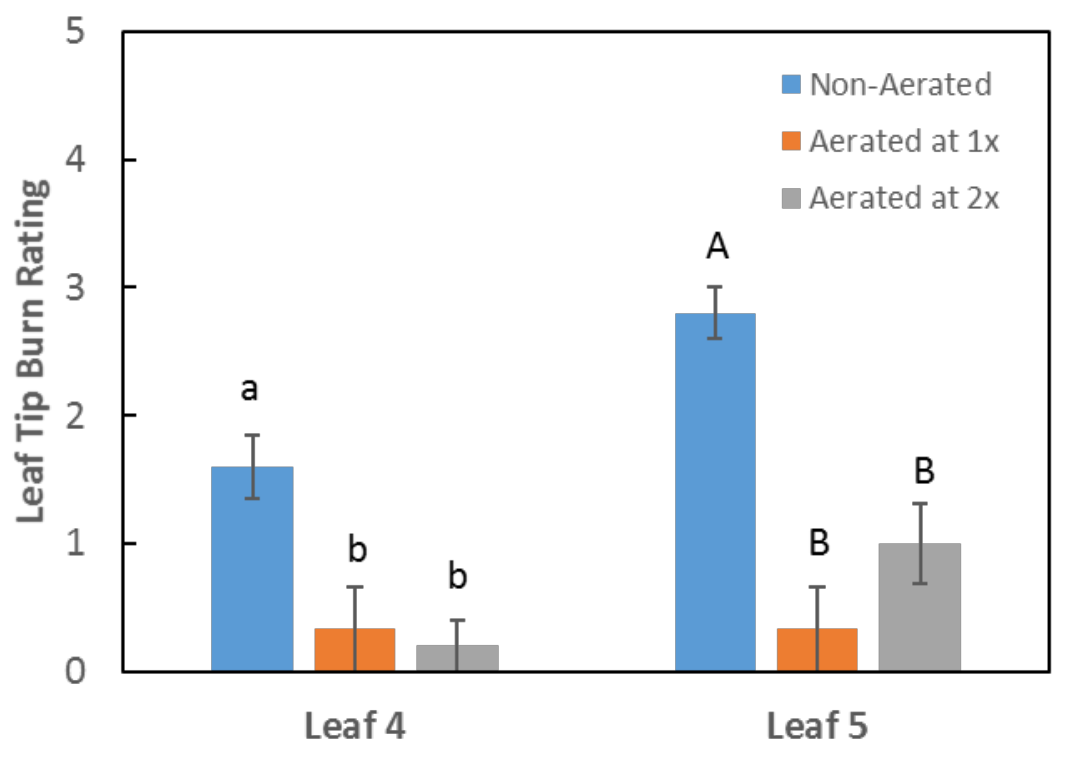


of both genotypes elongated at a steady-state growth rate or were approaching steady-state growth rates (Figs. 15 and 16). The growth rates of Node 1 and Node 2 roots were inhibited by low soil water potentials (Figs. 15 and 16), which will be discussed further in Chapter 3.

\section{DISCUSSION}

The present divided-chamber model system is unique from other split root systems previously described (Rostamza et al., 2013; Volkmar, 1997), because it includes an aeration system to alleviate possible complications with hypoxia in the inner chamber. Although aerating roots while in soil is not a common practice, it has been demonstrated that root growth in low water potential hydroponic solutions was also affected by oxygen availability (Verslues et al., 1998).

The divided-chamber study is also unique in its high reproducibility of stress imposition on the nodal roots through the uniform water potential media in the outer chamber. Because of the homogeneous soil environment, the roots achieve or approach a steady-state growth pattern (Figs. 15 and 16). Because of this steady-state growth pattern, detailed kinematic studies could be performed on the roots to further investigate the growth patterns and spatial resolution of nodal root growth maintenance in these two contrasting genotypes (Sharp et al., 1988; Sharp et al., 2004; Silk, 1984). 
Figure 15. Root growth rate of Node 1 roots from (A) B73 plants and (B) FR697 plants treated with well-watered soil (blue circles), -0.3 MPa soil (red circles), or $0.9 \mathrm{MPa}$ soil (green circles). Growth was steady state about 7 days post excavation and was inhibited by low soil water potentials. Growth rates were calculated from the root length increase marked along the side of the outer chamber divided by the time interval between markings. Data are means \pm SE ( $n$ $=3-7$ roots from $n=7$ plants). 


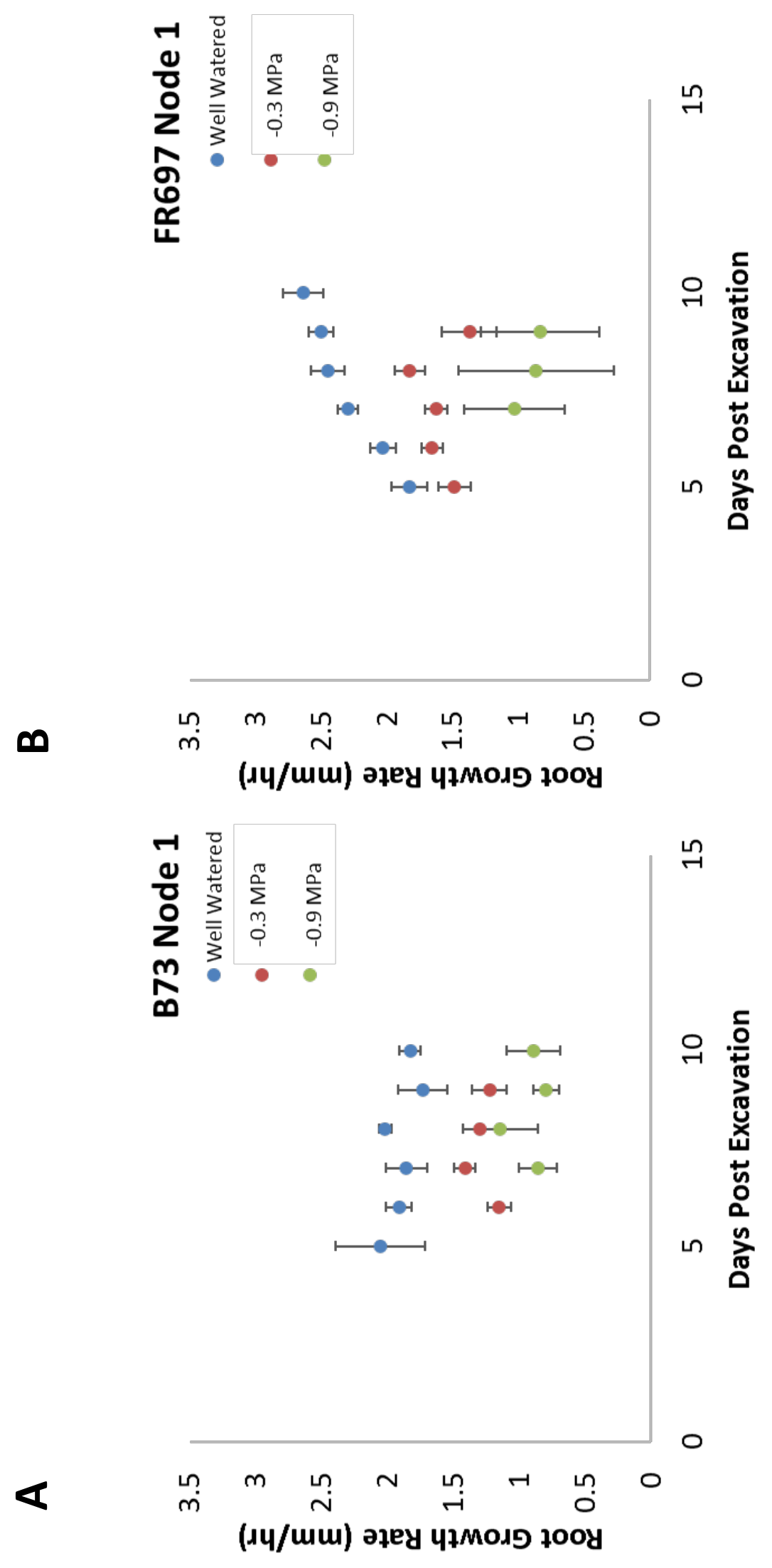


Figure 16. Root growth rate of Node 2 roots from (A) B73 plants and (B) FR697 plants growing in the divided-chamber system treated with well-watered soil (blue circles), -0.3 MPa soil (red circles), or -0.9 MPa soil (green circles). Growth was steady state and was inhibited by low soil water potentials. Growth rates were calculated from the root length increase marked along the side of the outer chamber divided by the time interval between markings. Data are means \pm SE ( $n$ $=3-7$ roots from $n=7$ plants). 


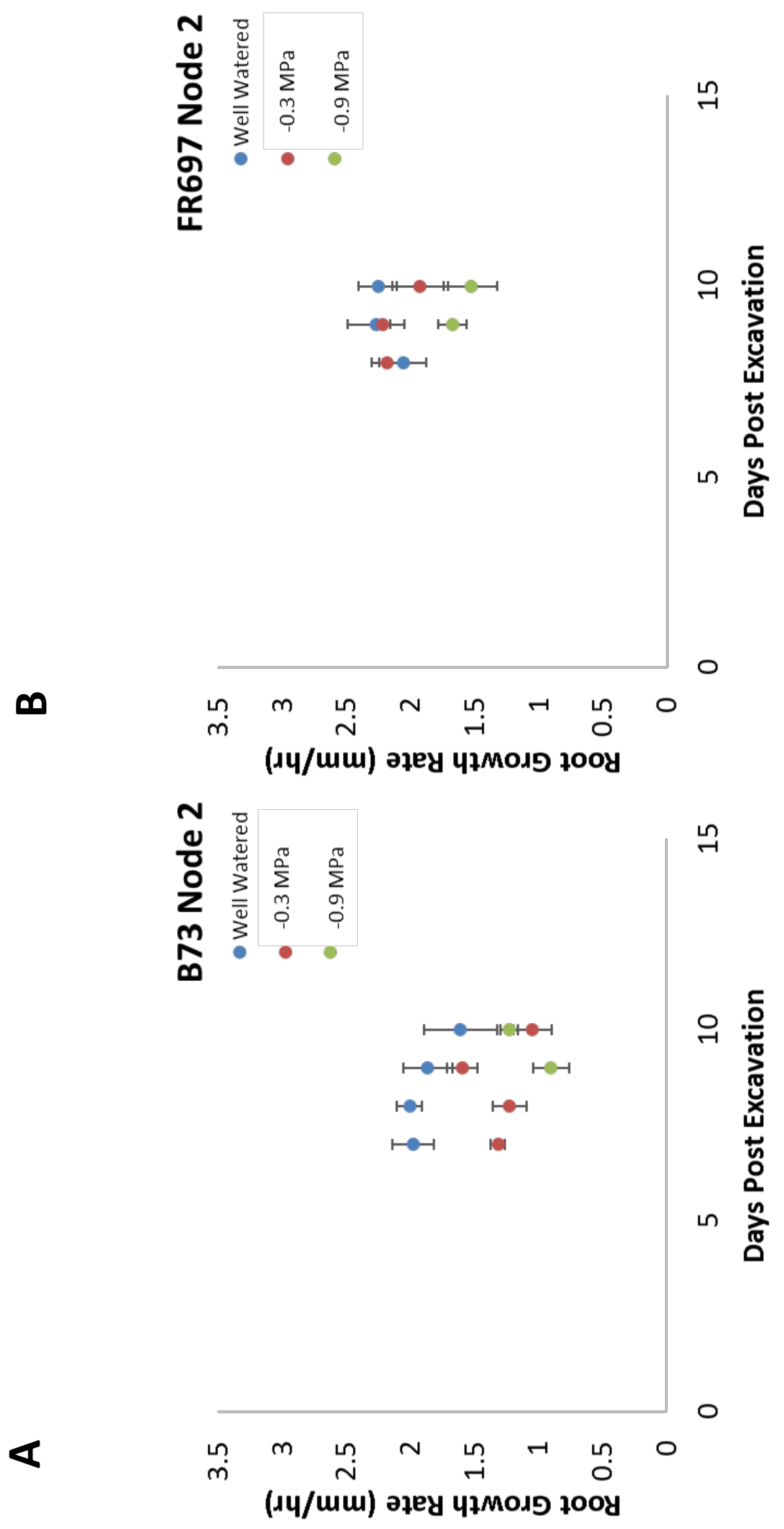


Because the outer walls of the nodal root chamber were clear, it was also possible to mark the growth rates of individual roots. With this resolution of data, it is possible to investigate the growth response differences between the distinct nodes of roots. Although the two nodes of roots emerged days apart, they both experienced identical uniform soil water potential treatments. Therefore, the system was used to study the growth responses of the two different nodes to a range of soil water potentials, which are described in more detail in Chapter 3.

Comparing genotypes in the present system is more accurate than traditional studies where water is withheld, because the stress imposed on the nodal roots was pre-determined. In traditional dry down studies, rate of stress imposition is significantly affected by leaf area and transpiration rate. Because leaf growth response to water stress and transpiration rates are genotypically diverse traits (Gholipoor et al., 2013; Kumari et al., 2004; Lu and Neumann, 1998), the severity and timing of water stress imposed on nodal roots may differ between genotypes and complicate the comparison.

Because the well-watered inner chamber supplies water to the shoot through the seminal and primary root systems, nodal root growth response to a wide range of soil water potentials may be tested without severe inhibition of shoot growth, which would prevent the formation of nodal roots (see Chapter 3 for more details). The present system also prevented the perception of water stress in the seminal and primary root systems, so that all water stress signals may be attributed to the low water potential soil on the nodal root system. 
The divided-chamber system could be used to study multiple stresses other than water stress, or the interaction of multiple stresses at one time. If the outer tube of transparency was replaced with PVC, it would be possible to study soil compaction stress and possibly heat stress by looping heating coils around the pots. Due to the highly controlled and reproducible growing conditions, this system would be suitable for studying a range of stress interactions in the nodal root system of maize and other monocots. 


\title{
CHAPTER 3
}

Maize nodal root growth response to water deficit in the divided-

\author{
chamber system
}




\section{ABSTRACT}

The divided-chamber model system was used to characterize nodal root growth responses to a range of soil water potentials under steady-state and reproducible conditions. Two contrasting genotypes, selected for differences in root growth response to water stress based on a previous study of the primary root, displayed similarly sensitive growth responses to $-0.3 \mathrm{MPa}$ soil, but different capacities to maintain high root tip water potential corresponding with different growth responses at low soil water potentials. Both genotypes maintained root tip water potentials in -2.0 MPa, despite the decreased soil water potential, suggesting a stress-induced response that enhances water transport to the root tip The difference in high tissue water potential maintenance was seen not only between the contrasting genotypes but also between the first two developmental nodes of roots. The divided-chamber system provides a powerful experimental approach to investigate the physiological mechanisms regulating nodal root growth responses to adverse soil conditions. 


\section{INTRODUCTION}

Nodal roots have a superior ability to maintain growth at low water potentials (Sharp and Davies, 1979; Westgate and Boyer, 1985), but little is known about the mechanisms enabling this growth maintenance. The following study was conducted to characterize nodal root growth responses over a range of low soil water potentials, utilizing the divided-chamber system previously described (see Chapter 2). Two contrasting genotypes for growth maintenance at low soil water potentials, inbred lines B73 and FR697 (Leach et al., 2011), and two contrasting developmental nodes, Node 1 and Node 2, were studied to understand the growth pattern differences between developmentally distinct nodes of roots in low soil water potentials.

Because the nodal roots are growing through dry soil in the present study, internal water sources through the phloem were hypothesized to be the source of water for the root tip based on previous studies (Boyer et al., 2010). Length that water travels to the growing tip affects the water potential of the tip, as increased length poses an increased resistance to water movement. Axial resistance in the transport phloem is proportional to length (De Schepper et al., 2013). In addition to resistance between the connecting elements, the transport phloem also feeds

the lateral root tip sinks along the root (De Schepper et al., 2013). The root tips of Node 1 and Node 2 are different distances from the shoot on a single plant. Because the internally sourced water would not be transported the same distance to maintain root growth of the different nodes, it was inadequate to compare the growth response of the two nodes from the same age of plants. 
It is also important to study growth rate of the nodal roots because several factors could influence the final length of the nodal axes at harvest. For example, if the genotypes differ in the rate of root emergence, length would look more sensitive in later emerging root varieties, particularly if the rate of emergence varied with decreased soil water potential or of the root tip water potential. When comparing average nodal axis length it is important to compare the stressed treatment to its well-watered control. Maize root growth under water stress conditions has been shown to be genotypically diverse in maize (Leach et al., 2011). The water stress tolerant response of interest in this study is the ability of a root to maintain growth relative to its well-watered root length or growth rate.

\section{Root tip water potentials: Limits to hydration}

A major factor influencing the growth of root tips is the drop in water potential of growing tissue, caused by growth-induced water potentials (Nonami and Boyer, 1987). Growth-induced water potential decline refers to the drop in cellular water potential when turgor declines due to the wall relaxation of the elongating cell. This drop in water potential serves to draw water into the growing tissue, as water thermo-dynamically flows from high to low water potentials (Nonami and Boyer, 1987).

The steep gradient of water potentials between the growing region and the mature tissue surrounding the enlarging cells may be attributed to a high hydraulic resistance (Nonami and Boyer, 1987). This gradient of tissue water 
potential was seen in a study by Sharp and Davies (1979) when growing maize nodal root tips were shown to have much lower water potentials than the mature root segments immediately behind the growth zone. The mature tissue, not expanding, is maintaining turgor and a higher water potential, assuming water is always flowing down the water potential gradient from the mature tissue into the growing, low water potential tip. At a certain proximity to the root tip, the meristem must be supplied water and solutes by diffusion through neighboring cells, because the xylem and sieve cells have not yet differentiated (Pritchard et al., 1996).

Exacerbating the growth-induced drop in water potential, xylem elements are immature near the apex and unable to conduct water as readily as the mature xylem elements. The earliest forming of these vessels are called protoxylem, and then progressively developing early metaxylem and late metaxylem with increasing wall strength and vessel diameter (Wenzel et al., 1989).

Wenzel et al. (1989) suggested that the maturation of the late metaxylem along the root axis can be delayed significantly in the earliest emerging nodal roots, as seen in two genotypes of maize and two genotypes of sorghum. The axes without late metaxylem are termed "immature axes," ranging in length up to $50 \mathrm{~cm}$ from the apex. In the maize cultivar Seneca Chief, late metaxylem did not develop until the plant was four weeks old with three "tiers" of roots, and similar results were found in four more varieties (Wenzel et al., 1989). 
In a second objective of the present study, the effect of tissue water potential on root growth maintenance in low soil water potentials was observed. The water potentials of the mature tissue immediately following the apical $20 \mathrm{~mm}$ of the nodal root tips were measured to elucidate the water potential gradient along the axis of the root.

\section{MATERIALS AND METHODS}

\section{Growth of plant materials}

Maize lines B73 and FR697 were grown as previously described (see Chapter 2). To adequately compare the two developmental nodes of roots, extended experiments of 9.5-10 days were conducted until the well-watered Node 2 roots grew to a similar length as the well-watered Node 1 roots in the initial 7 day experiments. The growth response of Node 2 roots from extended experiments was compared to the growth response of Node 1 roots in 7 day experiments. These extended experiments allowed the comparison of the two nodes at a similar distance from the stem, based on their well-watered growth patterns.

\section{Water potential measurements}

Soil and nodal root tip water potentials were measured using an isopiestic thermocouple pyschrometer. Apical $20 \mathrm{~mm}$ segments of the root tip were 
sampled to encompass the entire growing region of the nodal root tips.

Preliminary studies of the nodal root tip cell length profile indicated that the wellwatered nodal root growth zone was approximately $12 \mathrm{~mm}$ in length (data not shown). Mature tissue was left attached to the growing zone of the root tip to provide a source of water in the mature tissue and vascular elements for the growing tip (Nonami and Boyer, 1987). It was important to include mature tissue in the sample, because the tissues were still growing as the root tip was equilibrating during the reading. Without a source of water, the elongating cells in the root tip would lose turgor pressure, giving an erroneously low water potential measurement.

Mature tissue of the nodal root axes immediately following the apical 20 $\mathrm{mm}$ of the root tip (20-40 $\mathrm{mm}$ from the root apex) were also measured to observe any differences in growth-induced water potential gradients, resistance to water movement into the mature tissue, or loss of water from the root into the dry soil. The severe stress treatment (approximately -2.0 MPa) was used to study the mature nodal axis water potential of B73 and FR697 roots in Nodes 1 and 2.

\section{Measurement of root length and shoot growth}

Upon harvest, nodal root axes were measured by hand with a ruler. Root systems were washed and scanned for total nodal root system length using WinRHIZO software (Regent Instruments Inc.). Leaf area was measured after harvest using a LI-COR portable leaf area meter (model LI-3000A). Stem height 
was measured by hand with a ruler, and was defined as the length between the base of the crown, near the emergence site of Node 1 roots, to the collar of the last collared-leaf.

\section{Root growth rate calculation}

Elongation rate of the nodal roots was calculated from the length increase between the 7 day and extended 9.5-10 day harvest times, divided by the time increment.

During the experiment, the position of the root apices were marked as previously described (see Chapter 2). However, this data became harder to collect from plants treated with lower soil water potentials. The short roots did not all grow against the face of the outer chamber, and the difference between Node 1 and Node 2 roots was hard to differentiate from the limited markings. In addition, the roots that grew against the face of the outer chamber may have had restricted access to soil water due to differences in hydraulic contact with the soil surface. This may have created differences in growth rates for the roots along the face, which were not fully encased in soil.

\section{Statistical analyses}

Outliers of nodal root length were omitted using the Grubbs outlier test (Grubbs, 1950) after confirmation of normal distribution with the Shapiro-Wilk test 
(Shapiro and Wilk, 1965). One way analysis of variance (ANOVA) was analyzed between treatments in each genotype and node.

\section{RESULTS}

\section{Nodal root growth is sensitive to a mild drop in soil water potential}

The average length of nodal axes was significantly decreased by a mild drop in soil water potential (Fig. 1). Node 1 was most sensitive to treatment with $0.3 \mathrm{MPa}$, as both B73 and FR697 Node 1 roots were significantly inhibited by the treatment. After 10 days of treatment, Node 1 axes length of both genotypes reflected a similarly sensitive response to the mild water stress condition (Fig. $3 A)$.

Node 2 was slightly more resilient than Node 1, decreasing significantly in response to $-0.3 \mathrm{MPa}$ only in $\mathrm{B} 73$. There was no significant decrease in length of FR697 Node 2 roots in response to treatment with $-0.3 \mathrm{MPa}$ soil. Similar results were seen after 7 days of treatment with no significant decrease in FR697 Node 2 axes length but a significant decrease in B73 Node 2 axes length (Fig. 2A).

\section{Nodal root axes length is maintained between moderate and severe water stress conditions}

Despite the sensitive growth response to -0.3 MPa soil, nodal axis length of FR697 and B73 was maintained over a range of moderate to severe stress 
Figure 1. Nodal axis length of maize plants treated with various soil water potentials in the outer container of the divided-chamber system; the inner container was maintained in the well-watered condition throughout the experiments. (A) Node 1 from plants harvested on day 7 of treatment, and (B) Node 2 from plants harvested on day 10 of treatment shown for developmental length comparison. FR697 displays relative growth maintenance of nodal roots in response to low soil water potentials compared to B73. Within a genotype, means with different letters are significantly different, $p<0.025$. Nodal root tip (encompassing the growth zone) water potentials were higher in FR697 although both genotypes experienced similar soil water potentials (C, D). Node 2 displays relative growth maintenance relative to Node 1 in both genotypes. Water-stressed data are means \pm SE $(n=5$ 12 plants) from single or duplicate experiments. Well-watered control data are means \pm SE $(n=13-22$ plants) from three to four experiments. Arrows indicate the average axis length of roots experiencing soil water potential of approximately -2.0 $\mathrm{MPa}$ and the corresponding tissue water potential of the nodal roots from that treatment. 

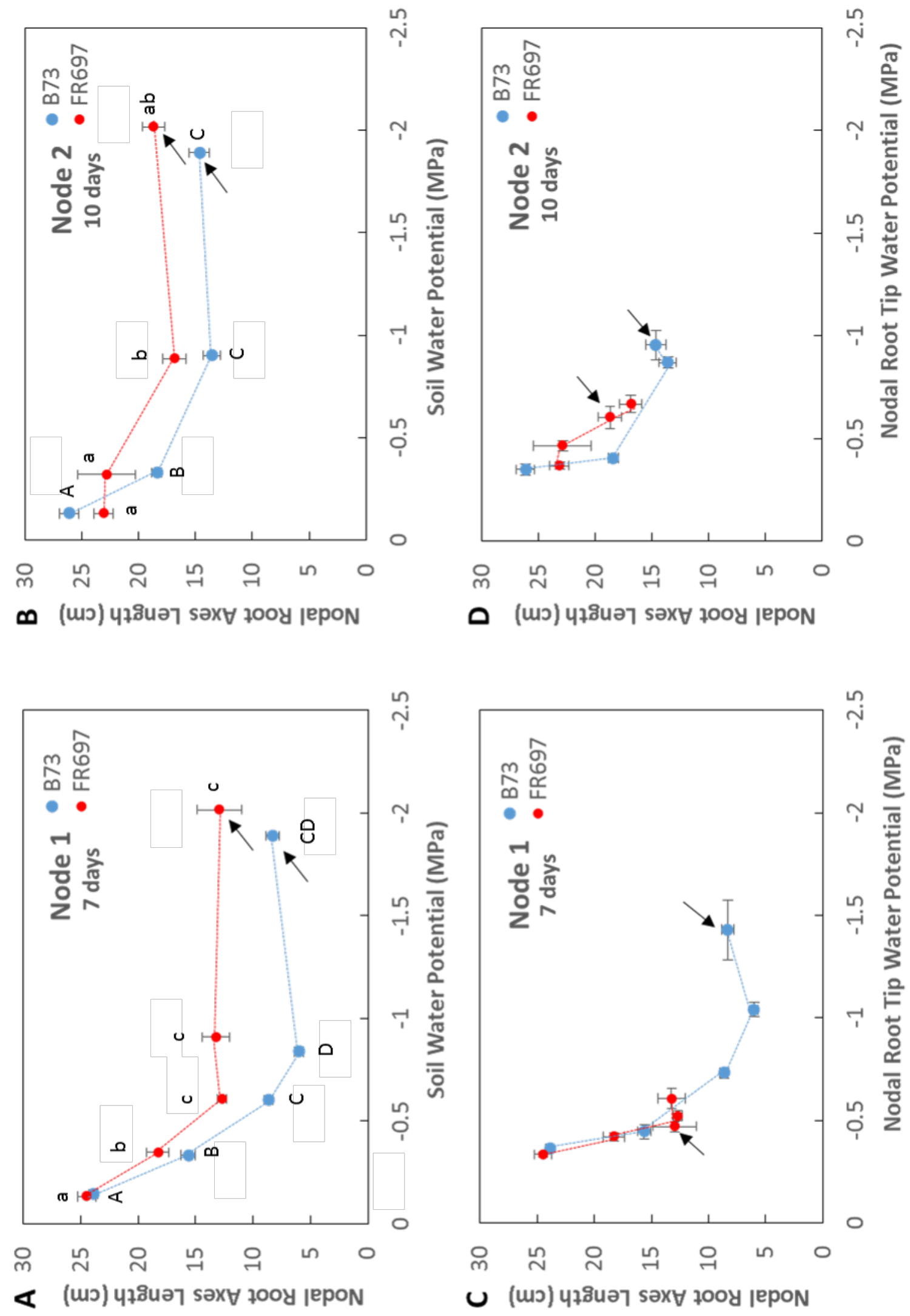
conditions (Fig. 1). In Node 1, growth was maintained between treatments with 0.6 MPa and -2.0 MPa soil for both genotypes (Fig. 1A). Growth of B73 Node 1 was slightly more inhibited in the $-0.9 \mathrm{MPa}$ soil treatment, but its growth in the more severe stress condition (-2.0 MPa) increased again to a length not significantly different from the $-0.6 \mathrm{MPa}$ treatment (Fig. 1A). In both genotypes, Node 2 length was not significantly inhibited between treatments with $-0.9 \mathrm{MPa}$ and -2.0 MPa soil (Fig. 1B). Moreover, growth of FR697 Node 2 roots was maintained between well-watered and -2.0 MPa treatments. Although growth was significantly inhibited with $-0.9 \mathrm{MPa}$ soil treatment, FR697 Node 2 root axis lengths were not significantly different between the well-watered and -2.0 MPa treatments.

\section{Root tip water potential corresponds with growth differences in severe water stress conditions}

Under moderate and severe water stress conditions (-0.9 MPa, -2.0 MPa), nodal root tip (encompassing the growth zone) water potentials were significantly higher in FR697 than B73, although both genotypes experienced similar soil water potentials (Fig. 1C, 1D). This trend was also seen in Node 2 after 7 days of treatment and in Node 1 after 10 days of treatment (Fig. 2B, 3B).

However, under mild stress conditions (-0.3 MPa) the tissue water potentials of FR697 and B73 were very similar in both nodes at both harvest times (Figs. 1C, 1D, 2B, 3B). Both genotypes maintained a relatively high tissue 
Figure 2. After 7 days of growth in the outer chamber, FR697 displays relative growth maintenance of Node 2 roots in response to low soil water potentials compared to B73 (A). Within a genotype, means with different letters are significantly different, $p<0.025$. Nodal root tip (encompassing the growth zone) water potentials were higher in FR697 although both genotypes experienced similar soil water potentials (B). Water-stressed data are means \pm SE $(n=5-12$ plants) from single or duplicate experiments. Well-watered control data are means \pm SE ( $n=17-22$ plants) from three to four experiments. Arrows indicate the average axis length of roots experiencing soil water potential of approximately $-2.0 \mathrm{MPa}$ and the corresponding tissue water potential of the nodal roots from that treatment. 

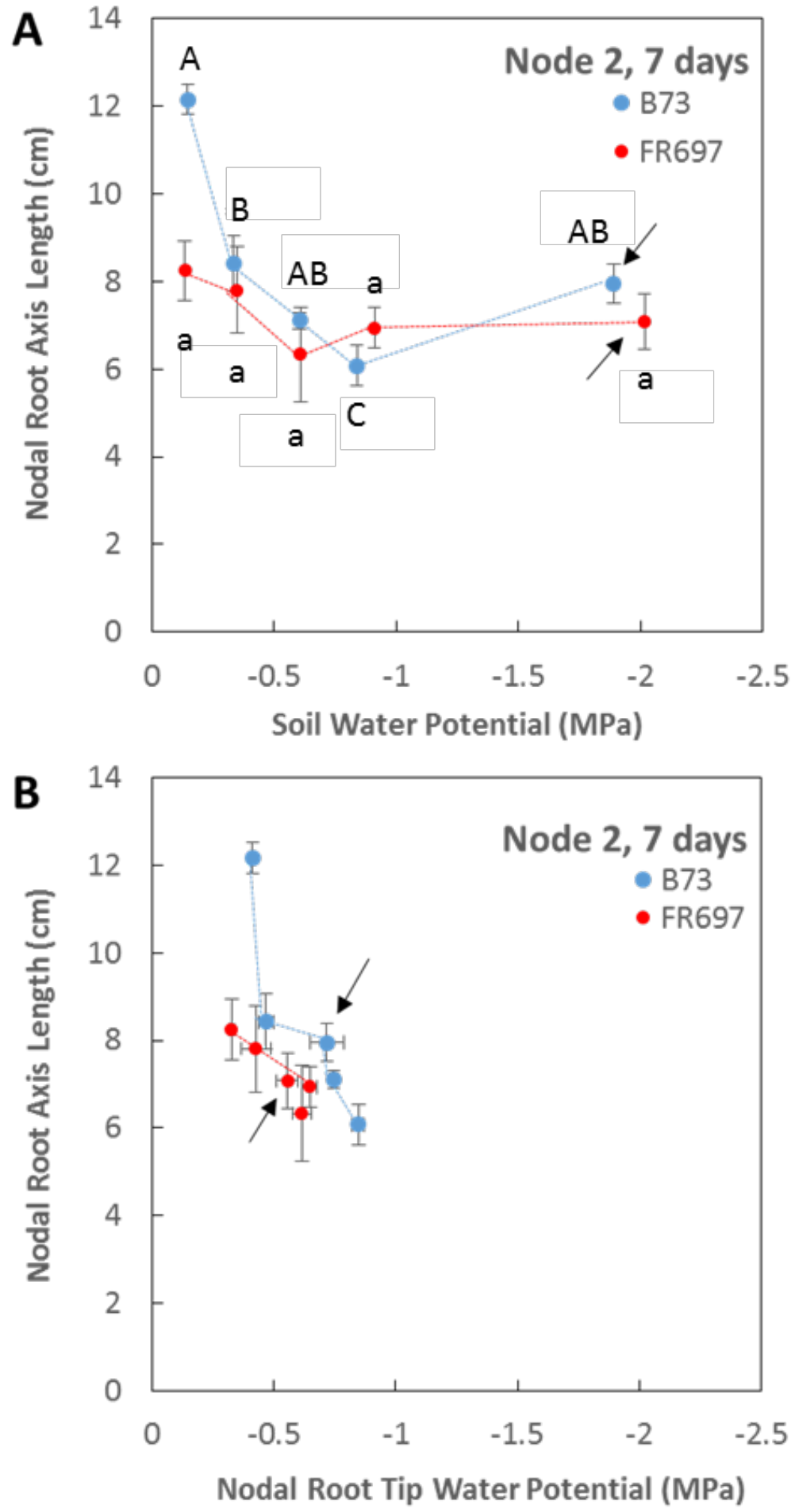
Figure 3. After 10 days of growth in the outer chamber, FR697 displays relative growth maintenance of Node 1 roots in response to low soil water potentials compared to B73 (A). Within a genotype, means with different letters are significantly different, $p<0.025$. Nodal root tip (encompassing the growth zone) water potentials were higher in FR697 although both genotypes experienced similar soil water potentials (B). Water-stressed data are means \pm SE $(n=6-11$ plants) from single or duplicate experiments. Well-watered control data are means \pm SE $(n=13-17$ plants $)$ from three to four experiments. Arrows indicate the average axis length of roots experiencing soil water potential of approximately $-2.0 \mathrm{MPa}$ and the corresponding tissue water potential of the nodal roots from that treatment. 

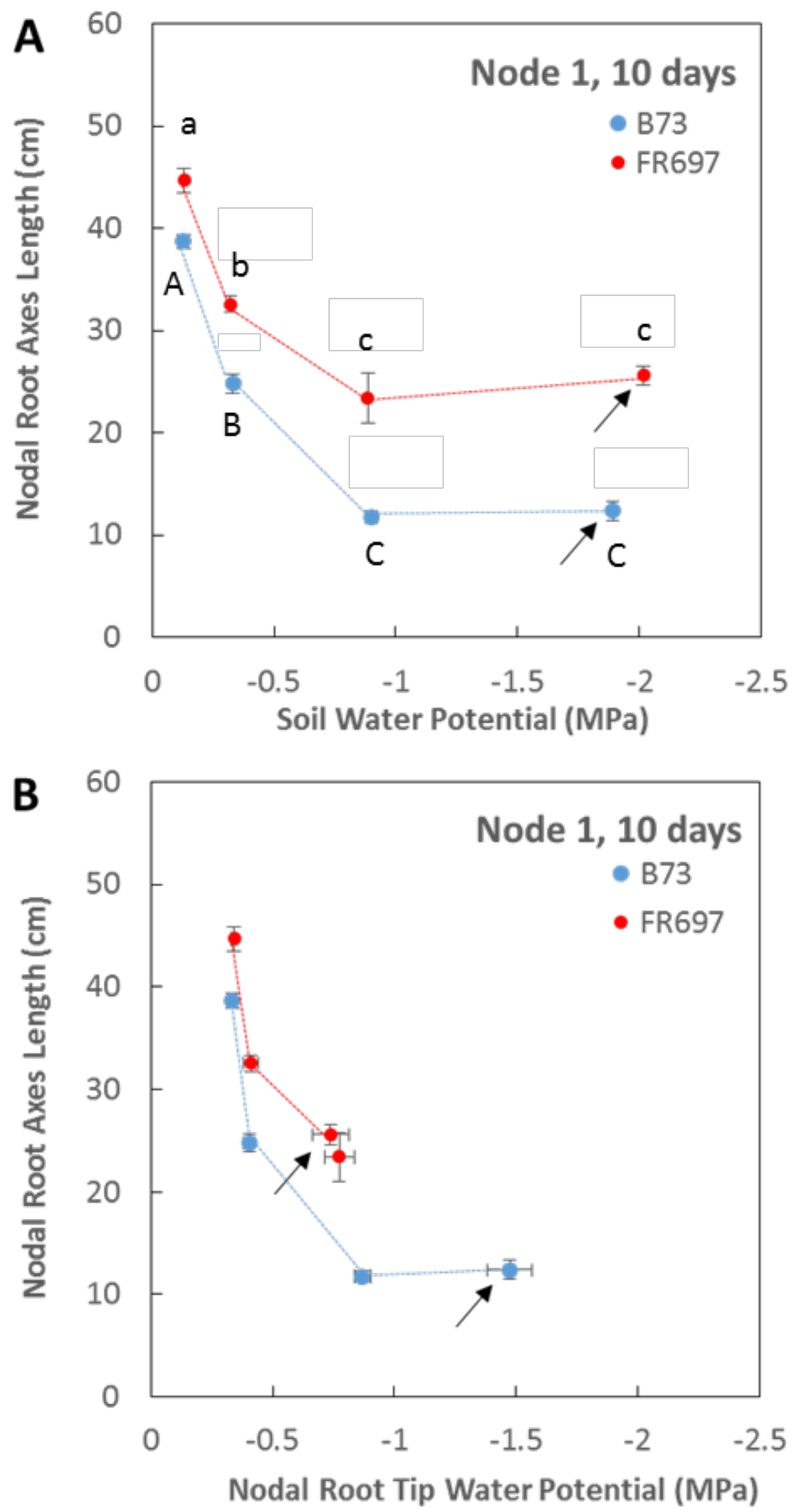
water potential in -0.3 MPa soil that was not significantly lower than their wellwatered root tip water potentials (Figs. 4, 5, 6).

\section{Relationship between soil water potential and root tip water potential}

In both genotypes the relationship between root tip water potential and soil water potential appeared linear, down to -0.9 MPa soil water potential (Figs. 4, 5, 6). More negative slopes indicate a more rapidly decreasing root tip water potential. Less negative slopes indicate a greater ability to maintain high root tip water potential despite the decreasing soil water potential. Due to the large range of soil water potentials between $-0.9 \mathrm{MPa}$ and $-2.0 \mathrm{MPa}$ that were unrepresented in the data, the second phase of the root tip-soil water potential relationship was connected with a dashed line to prevent interpretation of direct interpolation between these points (Figs. 4-6).

In both nodes FR697 displayed a greater ability to maintain high root tip water potentials relative to B73 (Fig. 4). The slope of root tip water potential decline became less negative in both genotypes after treatment with -2.0 MPa, causing a shift in the relationship between root tip and soil water potential (Figs. $4,5,6)$. This less negative shift was not substantial enough to maintain root tip water potential in Node 1 B73 roots after 7 or 10 days of treatment (Figs. 4A, 6). FR697 showed the greatest increase in root tip water potential in -2.0 MPa soil. In FR697 Node 1, treatment with -2.0 MPa soil produced a root tip water potential that was not significantly different from the well-watered root tip (Fig. 4A). B73 
Figure 4. Nodal root tip water potential as a function of soil water potential in the outer chamber. The relationship appeared linear in both genotypes at soil water potentials down to $-0.9 \mathrm{MPa}$. Steeper and more negative slopes indicate a more rapidly decreasing root tip water potential and development of greater water stress in the root tip while less negative slopes indicate a greater ability to maintain high root tip water potential despite the decreasing soil water potential. FR697 maintains higher root tip water potentials in both nodes of roots relative to B73. In addition, Node 2 roots maintain higher root tip water potentials under moderate to severe stress relative to Node 1 roots in both genotypes. Data were collected for Node 2 roots from plants that were three days older (B) when wellwatered Node 2 roots were a similar length to the Node 1 roots (A). Waterstressed data are means \pm SE $(n=4-11$ plants $)$ from single or duplicate experiments. Well-watered control data are means \pm SE $(n=10-18$ plants $)$ from three to four experiments. Within a genotype and node, means with different letters are significantly different, $p<0.025$. 

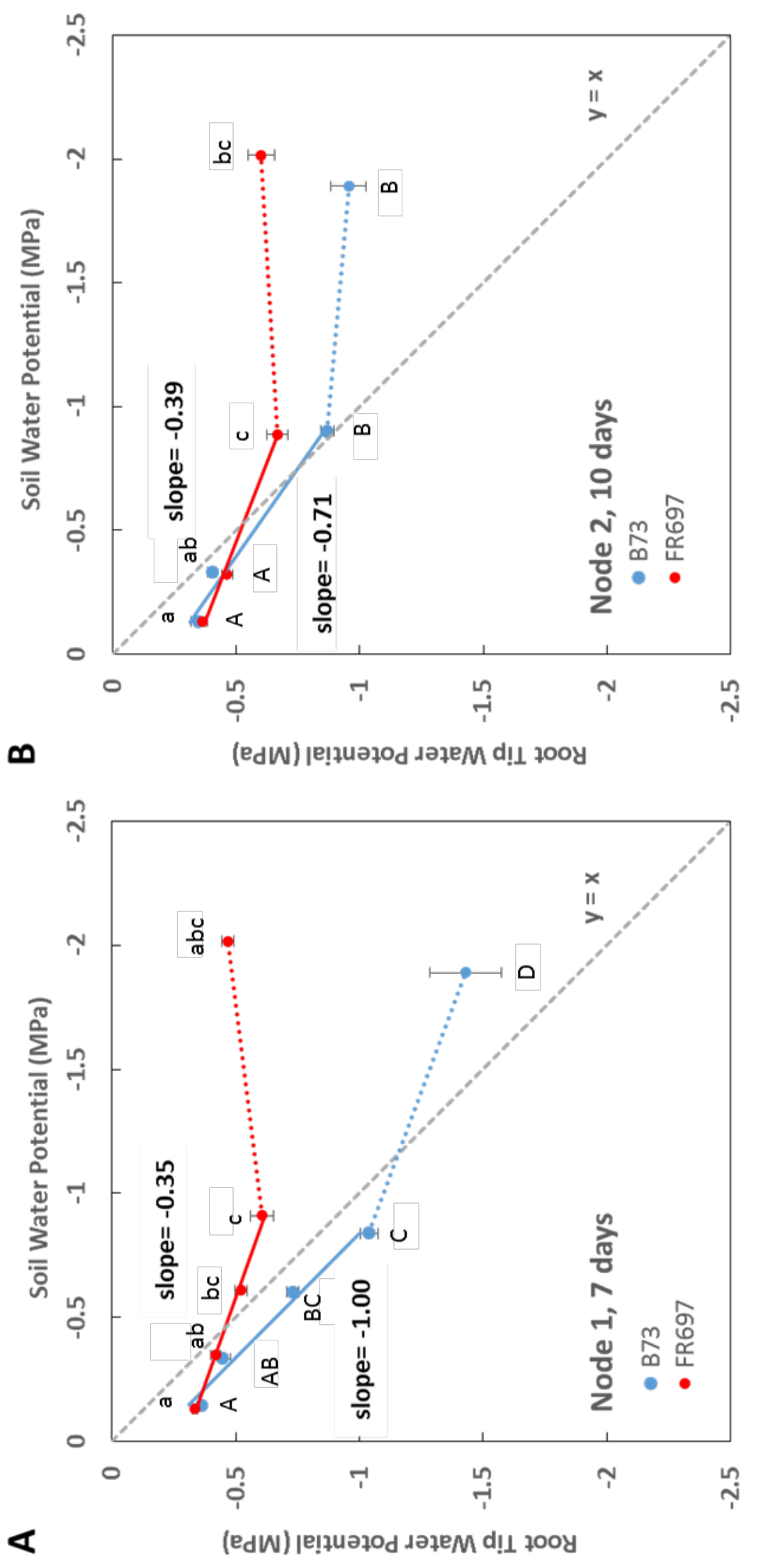
Figure 5. Nodal root tip water potential as a function of soil water potential in the outer chamber. The relationship appeared linear in both genotypes at soil water potentials down to $-0.9 \mathrm{MPa}$. Steeper and more negative slopes indicate a more rapidly decreasing root tip water potential and development of greater water stress in the root tip while less negative slopes indicate a greater ability to maintain high root tip water potential despite the decreasing soil water potential. Data represent Node 2 from plants harvested on day 7 of treatment. FR697 maintains higher root tip water potentials relative to B73. Water-stressed data are means \pm SE ( $n=4-11$ plants) from single or duplicate experiments. Well-watered control data are means \pm SE ( $n=10-18$ plants) from three to four experiments. Within a genotype, means with different letters are significantly different, $p<0.025$.

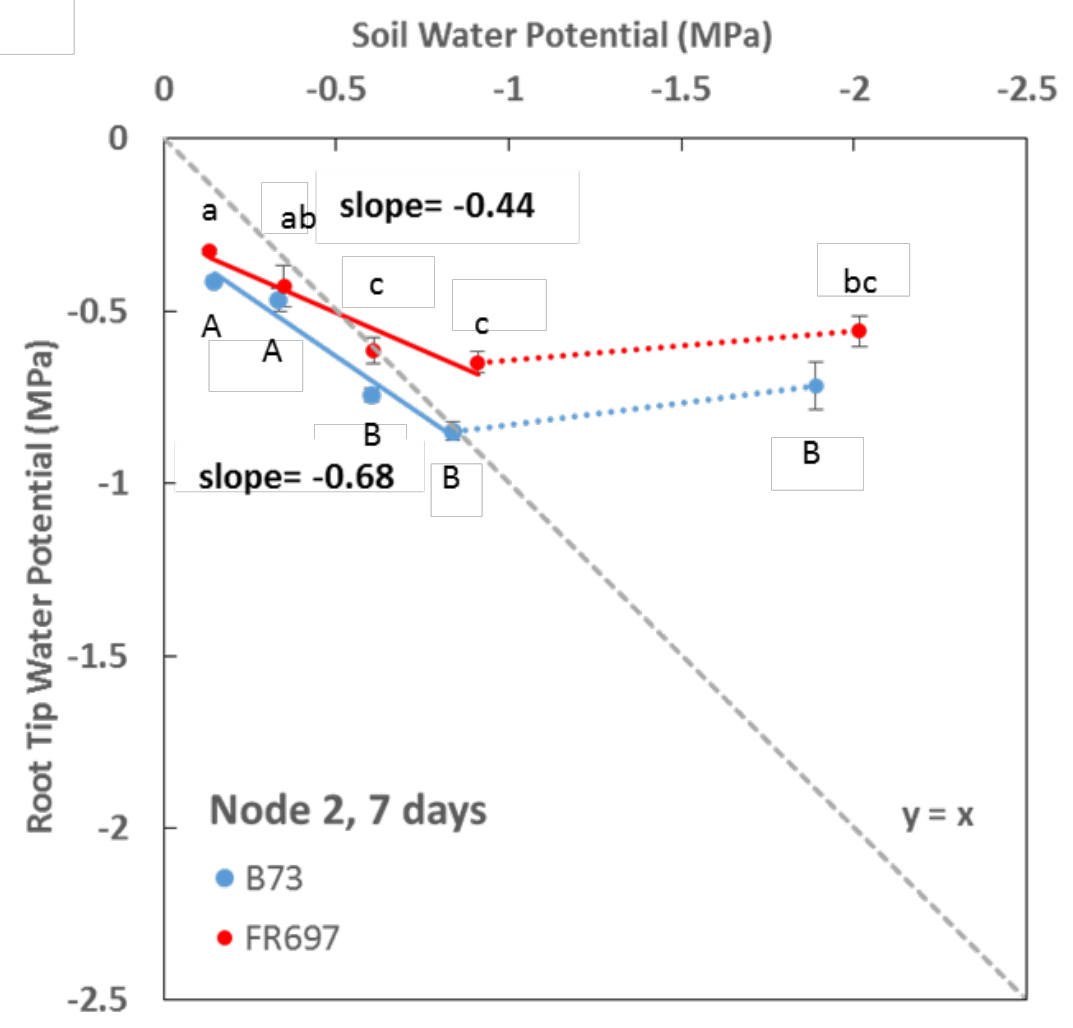


Figure 6. Nodal root tip water potential as a function of soil water potential in the outer chamber. The relationship appeared linear in both genotypes at soil water potentials down to $-0.9 \mathrm{MPa}$. Steeper and more negative slopes indicate a more rapidly decreasing root tip water potential and development of greater water stress in the root tip while less negative slopes indicate a greater ability to maintain high root tip water potential despite the decreasing soil water potential. Data represent Node 1 from plants harvested on day 10 of treatment. FR697 maintains higher root tip water potentials relative to B73. Water-stressed data are means \pm SE ( $n=4-11$ plants) from single or duplicate experiments. Well-watered control data are means \pm SE $(n=10-18$ plants $)$ from three to four experiments. Within a genotype, means with different letters are significantly different, $p<0.025$.

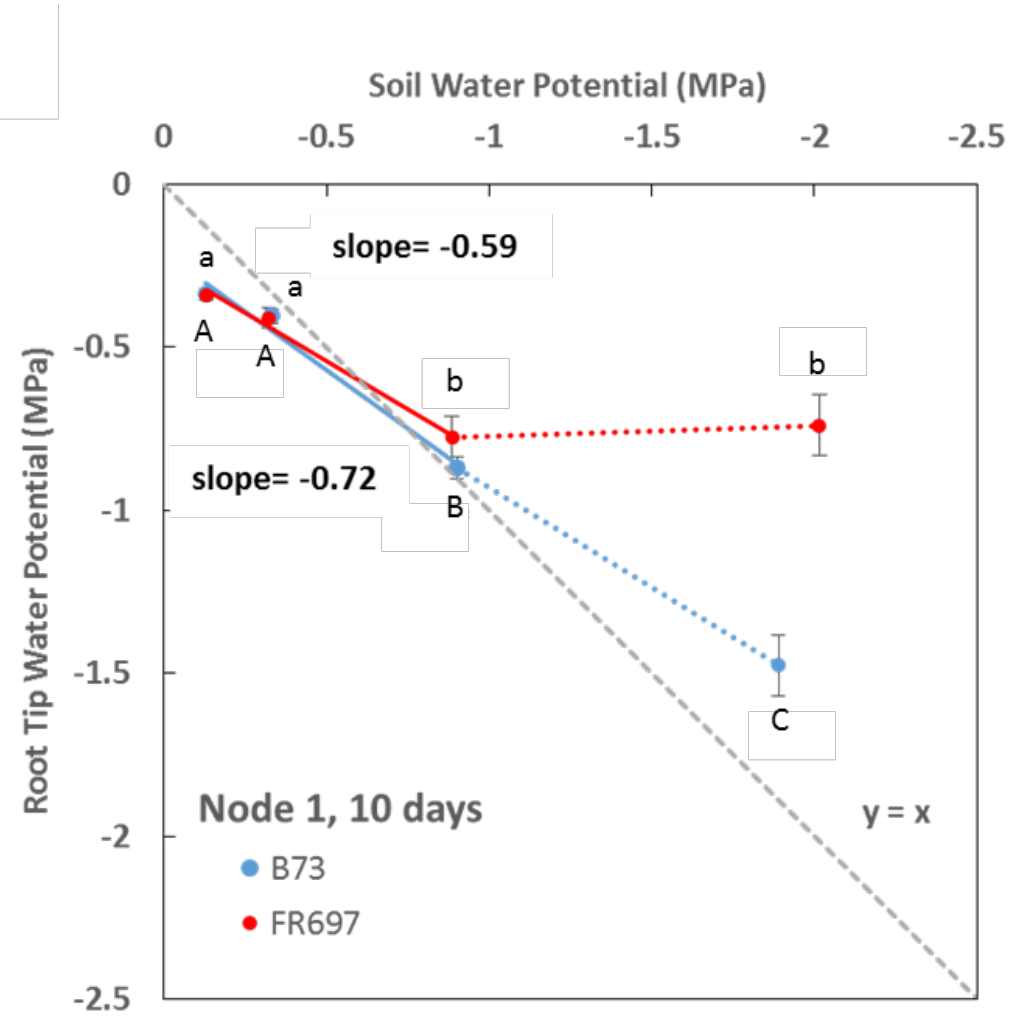


Node 2 root tip water potential was maintained after treatment with $-2.0 \mathrm{MPa}$ soil after 7 and 10 days (Figs. 4B, 5) relative to treatment with $-0.9 \mathrm{MPa}$. Root tip water potential was maintained over a larger range between $-0.3 \mathrm{MPa}$ and -2.0 MPa treatments in FR697 Node 2 after 7 and 10 day treatments (Figs. 4B, 5).

\section{Growth rate corresponds to root tip water potential}

Node 1 roots showed a decreased growth rate in response to mild stress (-0.3 MPa) in both genotypes (Fig. 8). FR697 Node 1 axes grew faster than B73 Node 1 axes, despite having similar tissue water potentials under mildly low soil water potentials (Fig. 8C). Under mild stress conditions (-0.3 MPa) B73 Node 2 roots also showed decreased growth rate, but FR697 Node 2 root growth was maintained (Fig. 8B, 8D).

Under moderate to severe water stress conditions (-0.9 MPa, -2.0 MPa), FR697 Node 1 maintained a higher root tip water potential than B73, which correlated with a faster growth rate (Fig. 8C). While the growth rate of B73 Node 1 continued to decrease with soil water potential, the growth rate of FR697 Node 1 increased (Fig. 8A). This increase in growth rate was correlated with an increase in root tip water potential.

Under moderate to severe water stress conditions (-0.9 MPa, -2.0 MPa) the difference in root tip water potentials between Node 1 and Node 2 was slight in FR697, corresponding to similar root growth rates between the nodes at equivalent developmental length (Fig. 9D). Under these same moderate to 
Figure 7. Nodal axis length as a function of nodal root tip water and soil water potential in the outer chamber. FR697 maintains growth of nodal roots and higher root tip water potentials in both nodes of roots relative to B73. In addition, Node 2 roots maintain growth and higher root tip water potentials relative to Node 1 roots in both genotypes. Data were collected for Node 2 roots from plants that were three days older (B) when well-watered Node 2 roots were a similar length to the Node 1 roots $(A)$. Water-stressed data are means $(n=4-11$ plants) from single or duplicate experiments. Well-watered control data are means $(n=10-18$ plants $)$ from three to four experiments. 

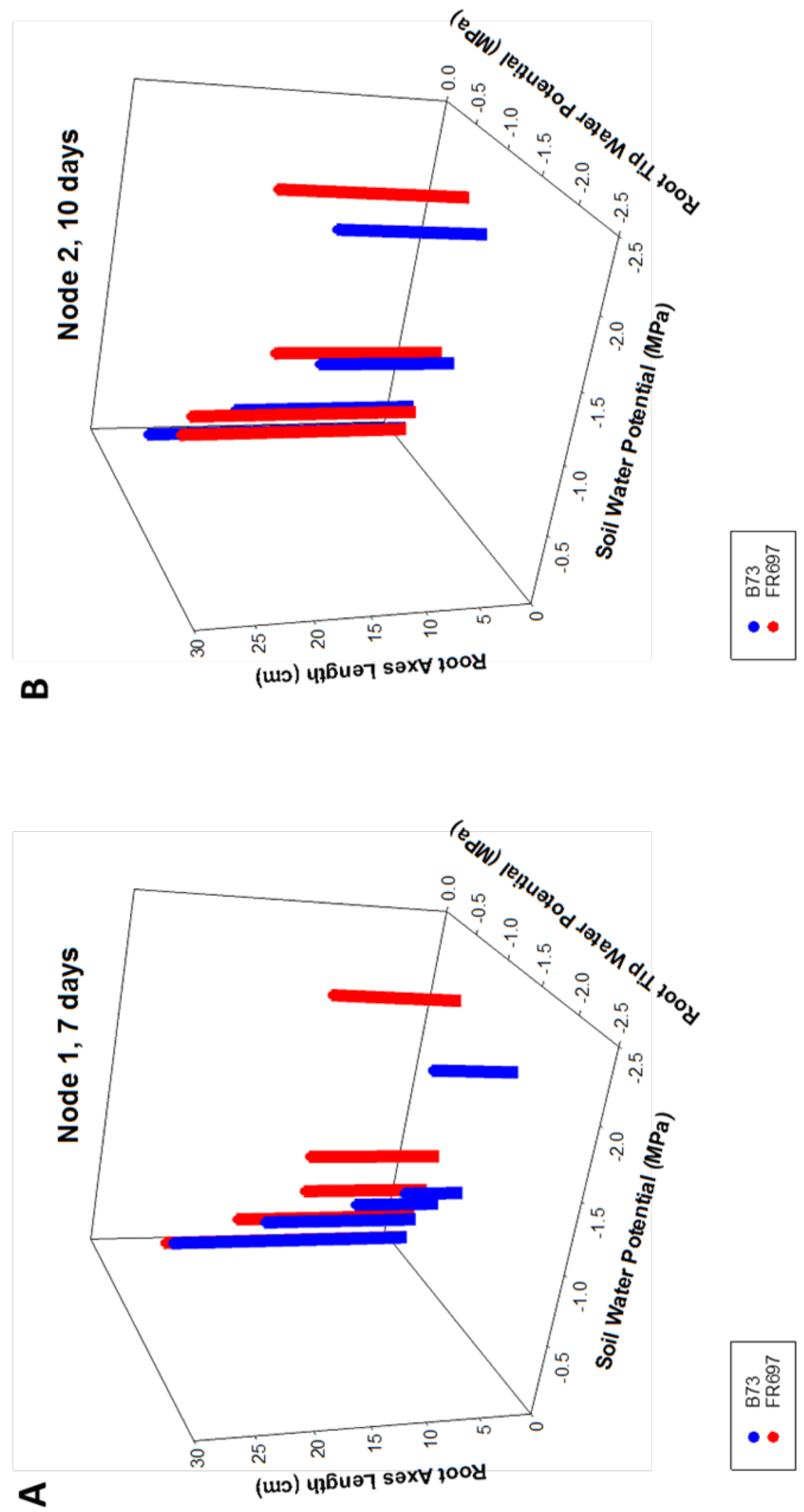
Figure 8. Nodal root growth rate as a function of soil water potential (A, B) and nodal root tip water potential (C, D). Data are differences of the mean nodal root axis length between plants harvested at 7 days and 10 days, divided by the time interval. Aside from FR697 Node 2, the nodal roots decreased growth dramatically in response to mild stress $(-0.3 \mathrm{MPa})$. The separate curves of genotypes plotted against soil water potential overlap when plotted against nodal root tip water potential. Arrows indicate the growth rates of roots experiencing soil water potential of approximately -2.0 MPa and the corresponding tissue water potential of the nodal roots from that treatment. 

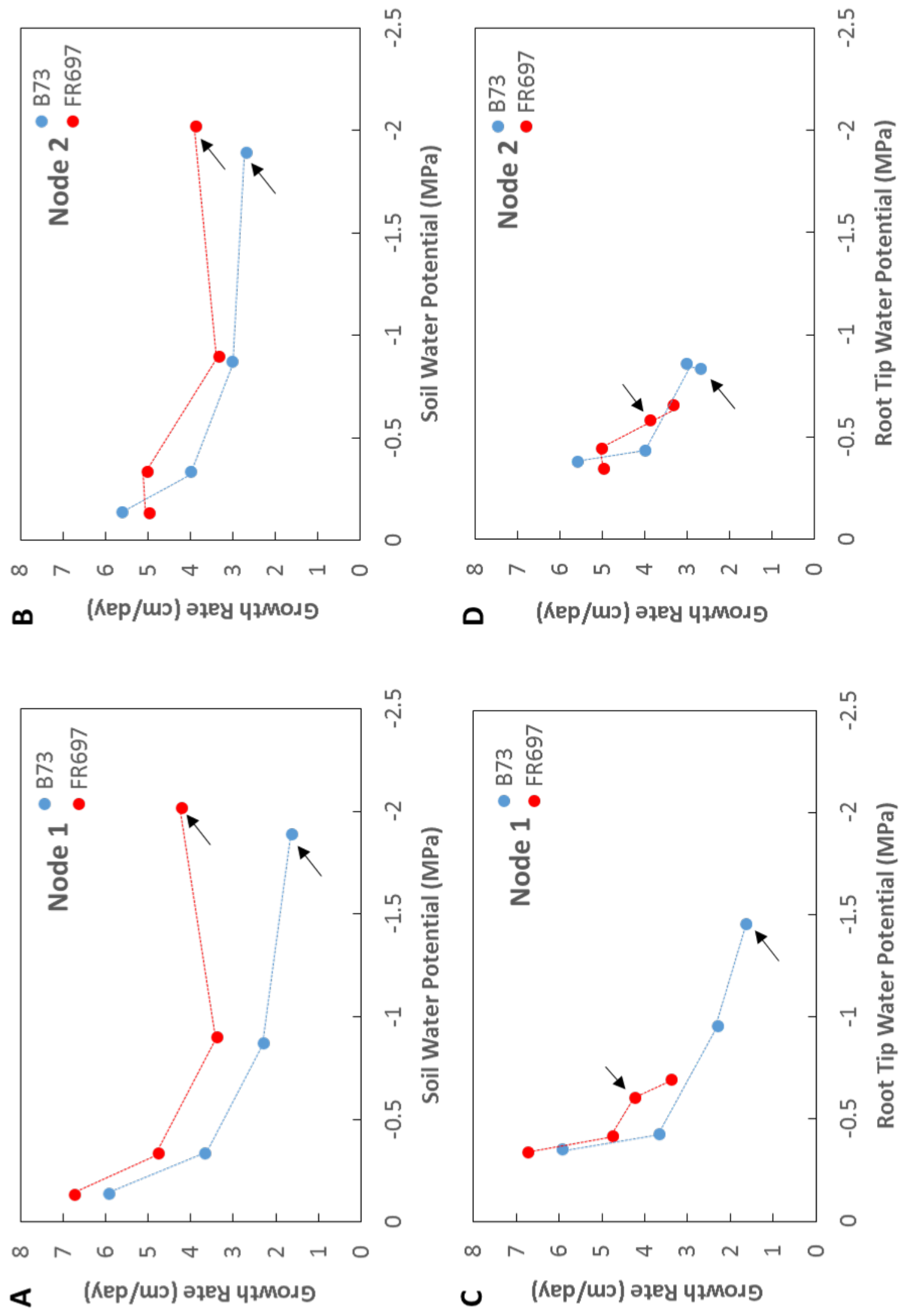
Figure 9. Nodal root growth rate as a function of soil water potential $(A, B)$ and nodal root tip water potential (C, D). Data are differences of the mean nodal root axis length between plants harvested at 7 days and 10 days, divided by the time interval. Node 2 of both genotypes maintains a faster growth rate in respect to its well-watered growth rate than Node 1, which decreases rapidly under mild water stress $(-0.3 \mathrm{MPa})$. The separate curves of Node 1 and Node 2 growth rates plotted against soil water potential overlap when plotted against nodal root tip water potential. Arrows indicate the growth rates of roots experiencing soil water potential of approximately $-2.0 \mathrm{MPa}$ and the corresponding tissue water potential of the nodal roots from that treatment. 

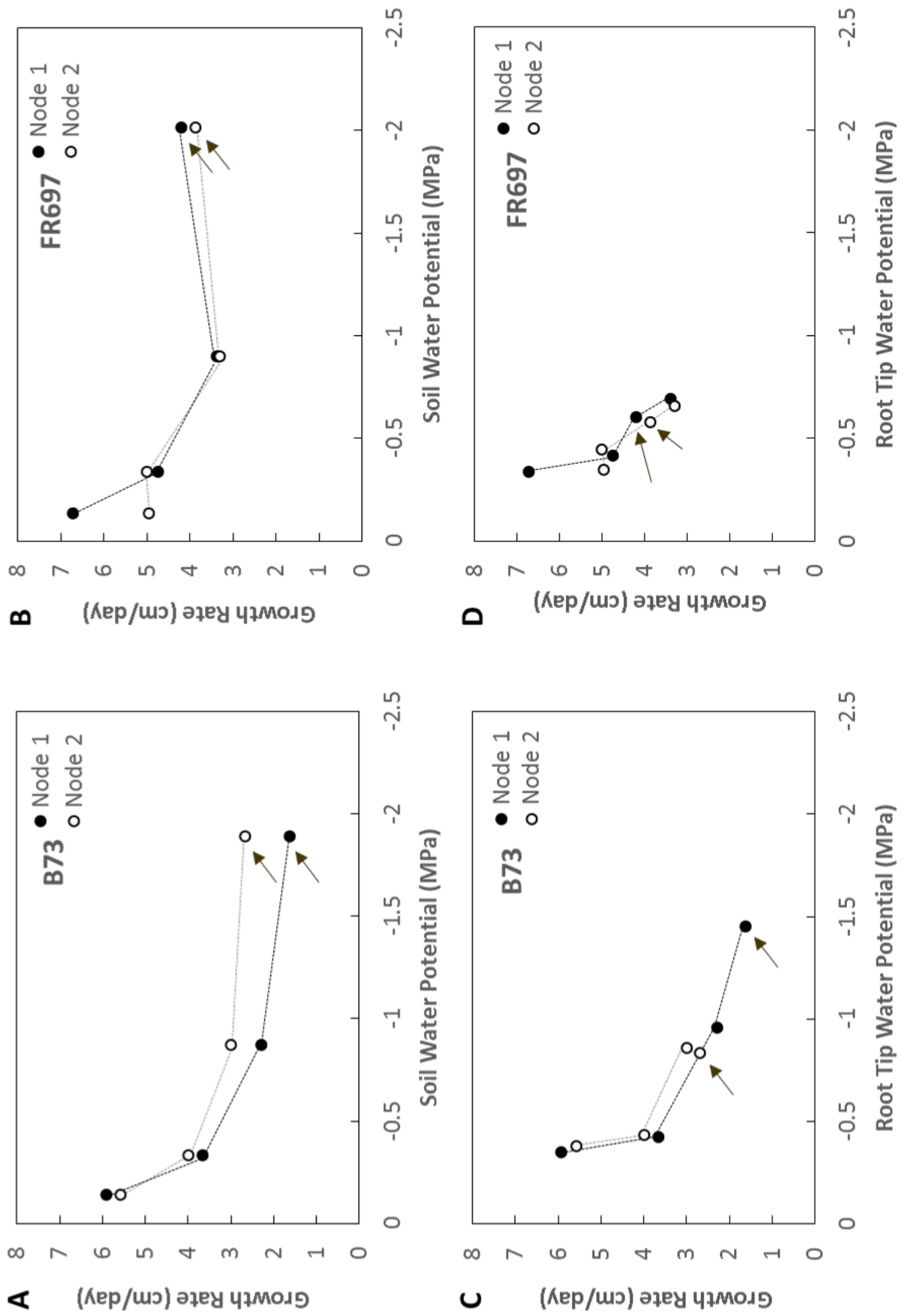
severe water stress conditions (-0.9 MPa, $-2.0 \mathrm{MPa}), \mathrm{B} 73$ Node 1 was unable to maintain a high root tip water potential relative to Node 2 . This correlated with a slight decrease in growth rate of B73 Node 1 relative to B73 Node 2 (Fig. 9C). Additionally, B73 and FR697 Node 2 roots were able to maintain growth and higher root tip water potentials at a greater distance from the shoot relative to Node 1 (Fig. 7).

\section{Water potential gradients into the root tip}

The plants displayed growth induced water potentials (Nonami and Boyer, 1987), as the axes had higher tissue water potentials in the mature regions and lower tissue water potentials in the growing regions (Figs. 10, 11, 12). This gradient may become steeper as the conductance of water into the root tip is decreased, suggesting a resistance to the flow of water from the hydrated mature tissue down the water potential gradient into the expanding root tip (Sharp and Davies, 1979).

FR697 well-watered root tips had less growth induced water potential gradients than well-watered B73 (Fig. 10C, D), but this small gradient between FR697 root tips and mature tissue was not reproduced. A growth induced water potential gradient was seen in FR697 roots in a follow up study (data not shown).

The water potential of the B73 Node 1 axis continued to decrease significantly over time and distance from the shoot, as the axis water potential after 10 days of treatment dropped so low it was no longer significantly different 
from the root tip water potential (Figs. 10A, 12A). In comparisons of tissue water potentials of the mature nodal root axis tissue (20-40 $\mathrm{mm}$ from the apex), FR697 Node 1 maintained a high root axis water potential in -2.0 MPa, which was not significantly different from the well-watered axis water potential (Fig. 10C). This trend was also seen after the longer 10 days of treatment with -2.0 MPa (Fig. 12B). In contrast, B73 Node 1 axis water potential was significantly lower than the well-watered axis water potential (Fig. 10A), and this water potential continued to drop after 10 days of treatment with -2.0 MPa (Fig. 12A).

When compared at equivalent length, FR697 Node 2 also maintained a high axis water potential, which was not significantly different from the wellwatered axis water potential (Fig. 10D). This trend was similar after the shorter 7 days of treatment with $-2.0 \mathrm{MPa}$ (Fig. 11B). B73 Node 2 was also able to maintain a high root axis water potential relative to the well-watered axis water potential after 10 days (Fig. 10B) and 7 days (Fig. 11A) of treatment.

\section{Shoot growth declines with low soil water potential in the outer chamber}

As soil water potential in the outer chamber decreased, the total leaf area in 7 and 10 day old plants also decreased significantly in both genotypes (Fig. 13). The decrease in total leaf area corresponded with treatments where nodal root growth was maintained at both harvest times (Figs. 1A, 1B, 2A, 3A, 13). The difference in leaf area of both genotypes in response to low soil water potential was caused by inhibition of leaf area of later developing leaves (Fig. 13), which 
Figure 10. Comparison of tissue water potentials of nodal root tips $(0-20 \mathrm{~mm}$ from the apex, encompassing the growth zone) and mature nodal root axis tissue (20-40 $\mathrm{mm}$ from the apex) from plants treated with well-watered or very dry (approximately -2.0 MPa) soil in the outer chamber. B73 Node 1 (A) and Node 2 (B) do not maintain nodal root water potentials in the apical tip or the axis tissue. FR697 maintains a higher root tip and root axis water potential in Node 1 (C) and Node 2 (D) tissues grown at approximately -2.0 MPa soil water potential. Data were collected for Node 2 roots from plants that were three days older $(B, D)$, when Node 2 roots were a similar length to the Node 1 roots $(A, C)$. Data are means \pm SE $(n=4-6$ plants $)$ from single experiments. Data from Node $1(A, C)$ and Node $2(B, D)$ were analyzed separately for statistical significance. Means with different letters are significantly different, $p<0.025$. 

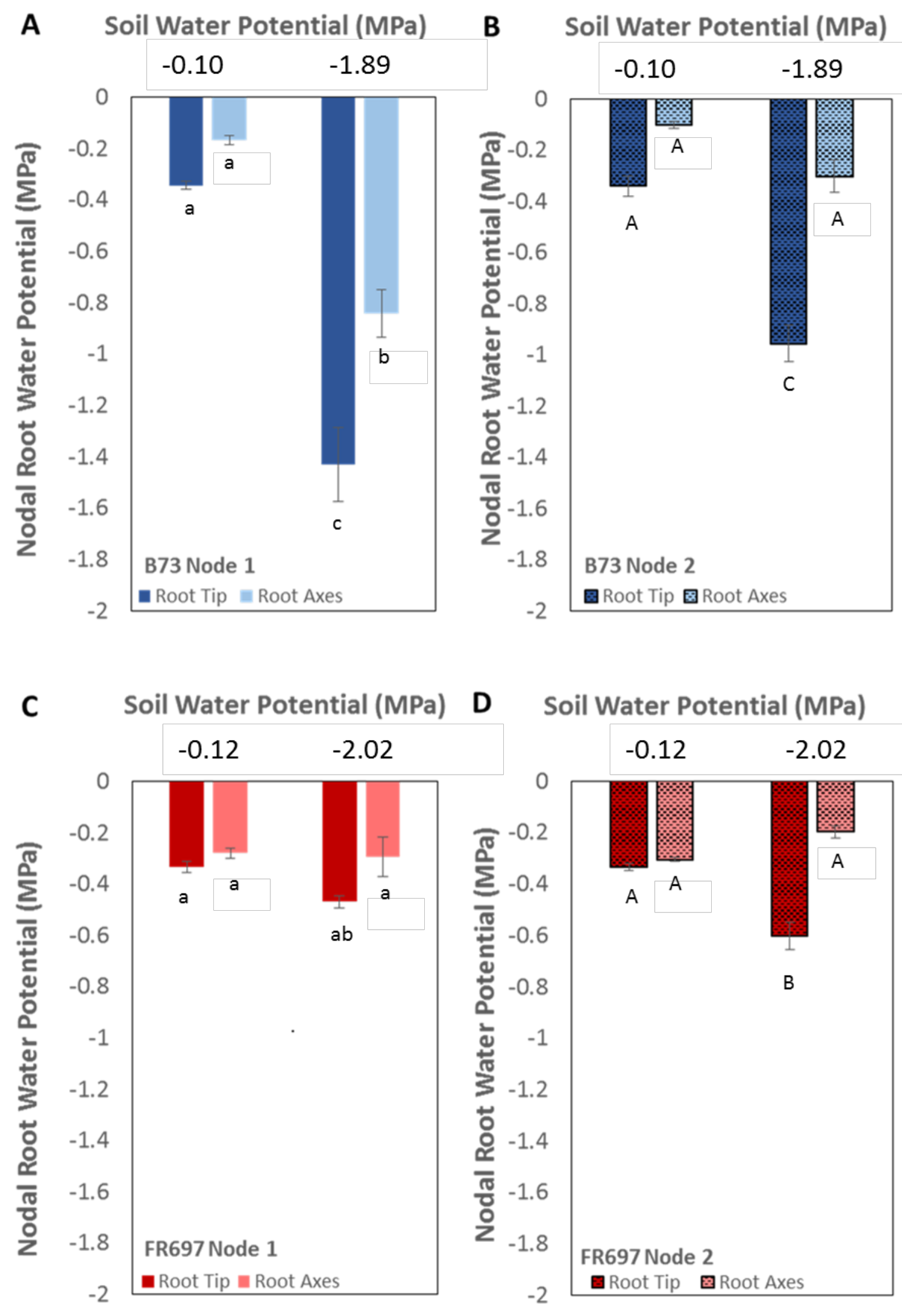
Figure 11. Comparison of tissue water potentials of growing nodal root tips (0-20 $\mathrm{mm}$ from the root apex, encompassing the growth zone) and mature nodal root axis tissue (20-40 $\mathrm{mm}$ from the apex) from plants treated with well-watered or very dry (approximately -2.0 MPa) soil in the outer chamber. Data represent Node 2 from plants harvested on day 7 of treatment. When compared with B73 (A), FR697 (B) maintains higher root tip water potentials in Node 2 tissues grown in approximately -2.0 MPa soil water potential. Data are means \pm SE $(n=3-6$ plants) from a single experiment. Data from Node $2(A, B)$ were analyzed together for statistical significance. Means with different letters are significantly different, $p<0.025$.
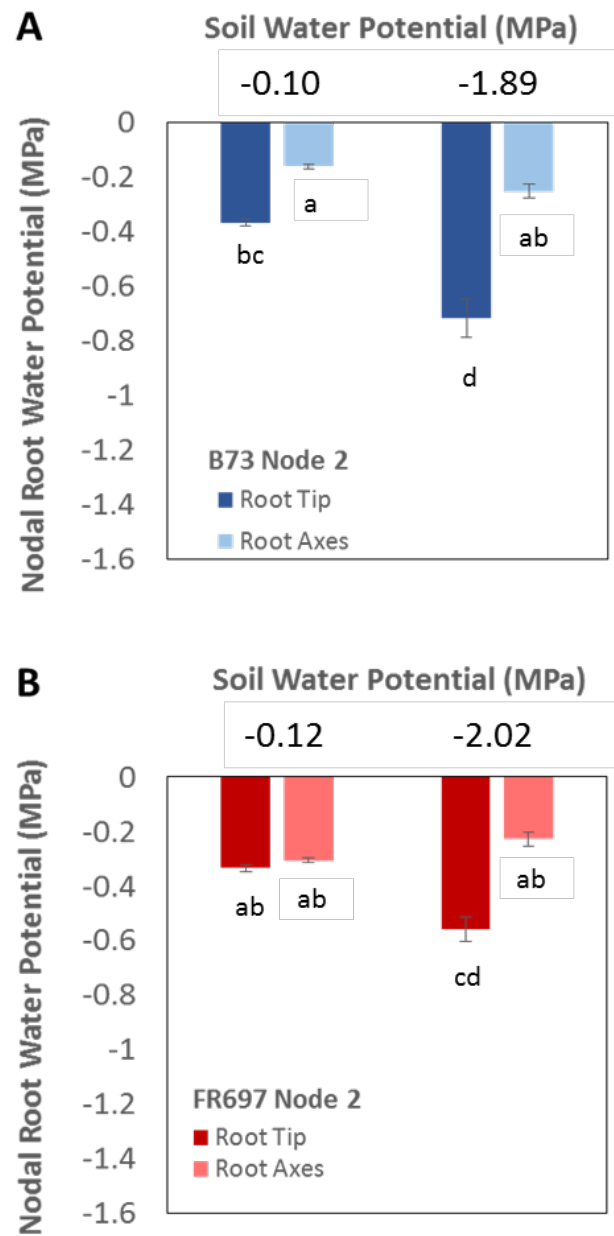
Figure 12. Comparison of tissue water potentials of nodal root tips $(0-20 \mathrm{~mm}$ from the root apex, encompassing the growth zone) and mature nodal root axis tissue (20-40 $\mathrm{mm}$ from the apex) from plants treated with well-watered or very dry (approximately -2.0 MPa) soil in the outer chamber. Data represent Node 1 from plants harvested on day 10 of treatment. When compared with B73 (A), FR697 (B) maintains a higher root tip and root axis water potential in Node 1 tissues grown in approximately -2.0 MPa soil water potential. Data are means \pm SE $(n=4-6$ plants) from a single experiment. Data from Node $1(A, B)$ were analyzed together for statistical significance. Means with different letters are significantly different, $\mathrm{p}<0.025$.
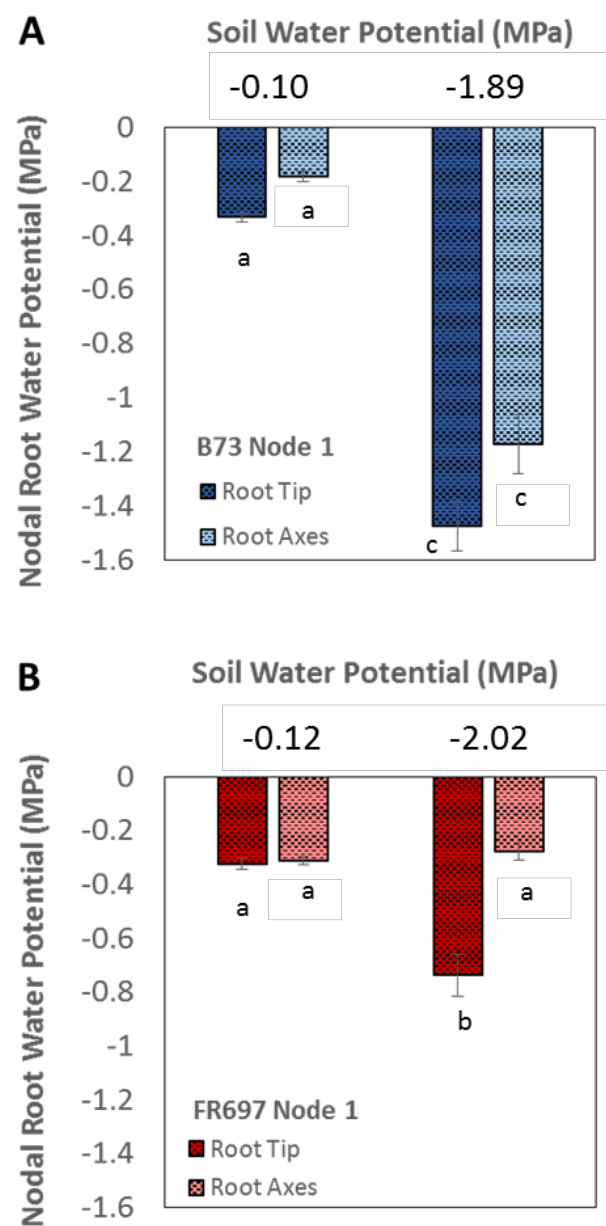
Figure 13. B73 (A, C) and FR697 (B, D) leaf area as a function of soil water potential in the outer chamber. A significant decrease in total leaf area can be seen in both genotypes, whereas leaves 1-3 maintain their leaf area. Waterstressed data are means \pm SE ( $n=5-12$ plants) from single or duplicate experiments. Well-watered control data are means \pm SE $(n=13-22$ plants $)$ from three to four experiments. Statistical significance is indicated on total leaf area only. Means with different letters are significantly different, $p<0.025$.
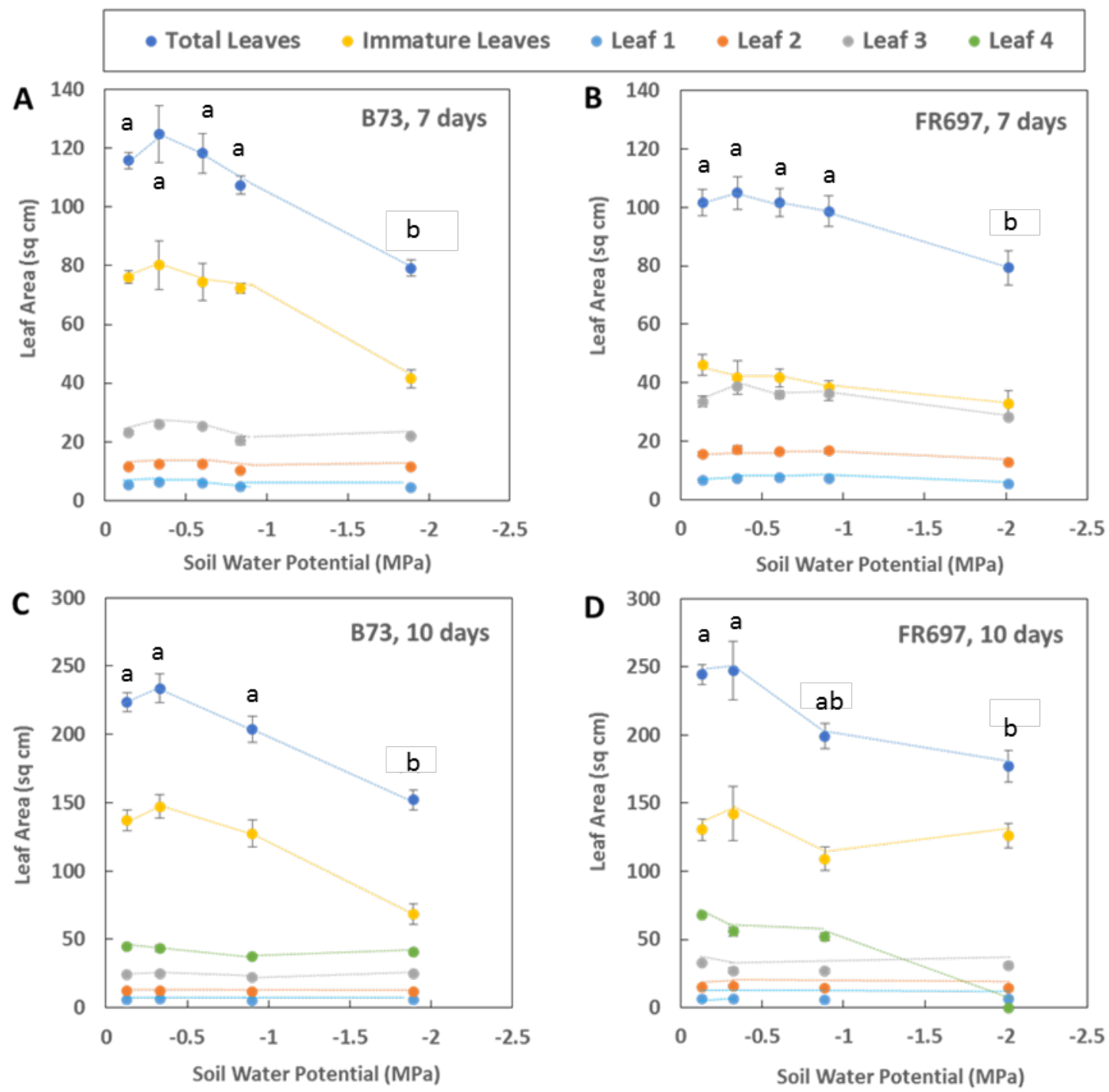
had expanded while the first and second nodes of roots emerged. Leaf dry weight was also measured and showed similar trends. Stem height was also significantly decreased in B73 after 10 days of treatment. However, stem height in FR697 was not significantly inhibited by low water potential soil (Fig. 14).

\section{Seminal and primary root system growth increases with low soil water potential in the outer chamber}

Seminal root axes length did not significantly increase under mild or severe water stress conditions in either genotypes after 7 or 10 days of treatment. After 10 days of treatment with severely low soil water potential (-2.0 $\mathrm{MPa}$ ), the seminal root system (including seminal lateral roots and axes) proliferated significantly compared to well-watered plants. Because there was no significant increase in the seminal root axes length, this increase in length was comprised of an increase in seminal lateral root growth (Fig. 15).

Primary root axes length did not significantly increase under mild or severe water stress conditions in either genotypes after 7 or 10 days of treatment. After 10 days of treatment with moderate or severe water stress $(-0.9$, -2.0 MPa), the primary root system of FR697 (including primary root laterals and axis) proliferated significantly compared to well-watered plants. The primary root system of B73 also significantly increased at $-0.9 \mathrm{MPa}$ after 7 and 10 days of treatment. Because the primary root axes length did not change significantly, this increase in length was comprised of an increase in lateral root growth on the primary root axis (Fig. 16). 
Figure 14. B73 and FR697 stem height as a function of soil water potential in the outer chamber. Plants were grown for 7 days $(A)$ or 10 days (B) in the outer chamber before harvest. When treated with low water potential soil, stem height decreased significantly in B73 after 10 days of treatment. However, stem height in FR697 was not significantly inhibited by low water potential soil. Water-stressed data are means \pm SE ( $n=4-12$ plants) from single or duplicate experiments. Wellwatered control data are means \pm SE $(n=13-22$ plants $)$ from three to four experiments. Means with different letters are significantly different, $p<0.025$. Within a genotype, means with the same letter are not significantly different, $p>0.05$.
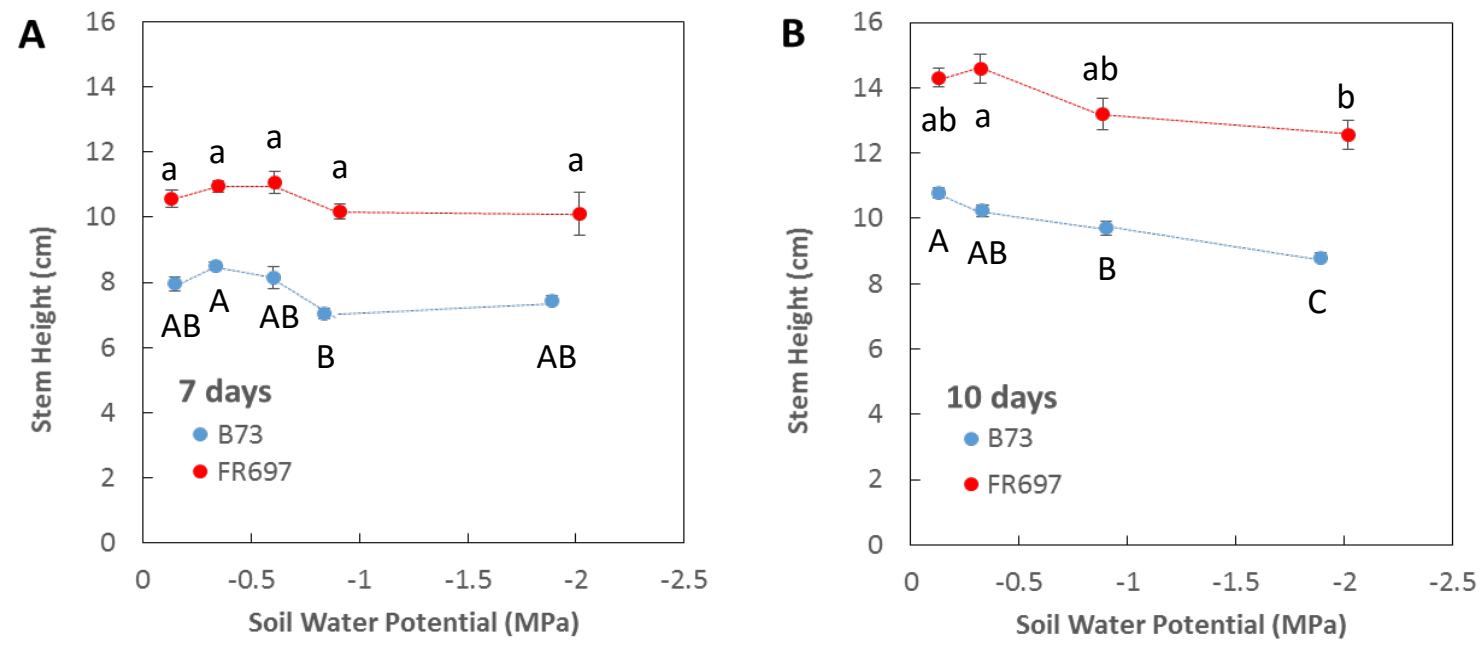
Figure 15. B73 and FR697 seminal root axes length (A, B) and seminal root system length, including lateral roots, (C, D) as a function of soil water potential in the outer chamber. Plants were grown for 7 days $(A, C)$ or 10 days $(B, D)$ in the outer chamber before harvest. Seminal root axes length did not significantly increase under mild or severe water stress in either genotype after 7 or 10 days of treatment. After 10 days of treatment with severe water stress $(-2.0 \mathrm{MPa})$, the seminal system proliferated significantly compared to well-watered plants. Because the axes length did not change significantly, this increase in length was comprised of an increase in lateral root growth. Water-stressed data are means \pm SE ( $n=5-12$ plants) from single or duplicate experiments. Well-watered control data are means \pm SE ( $n=12-22$ plants) from three to four experiments. Within a genotype, means with different letters are significantly different, $p<0.025$. Means with the same letter are not significantly different, $p>0.05$. 


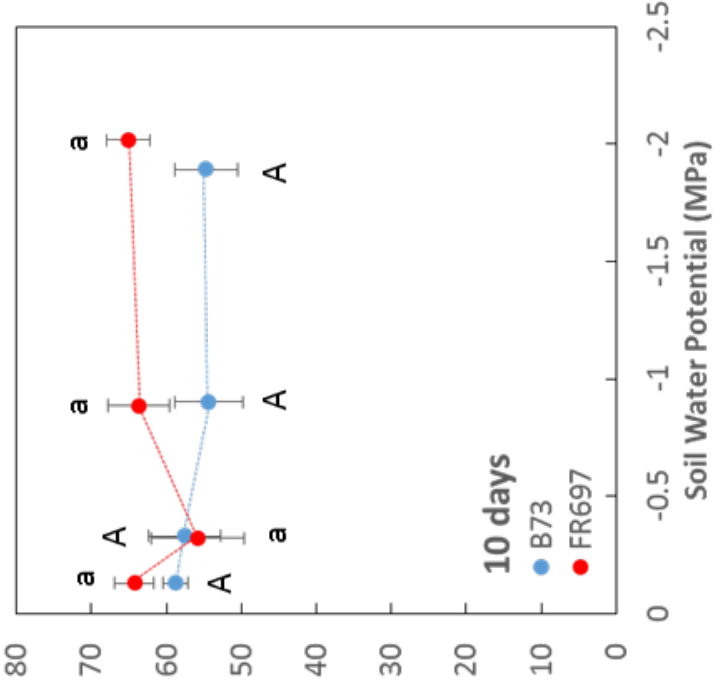

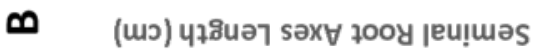

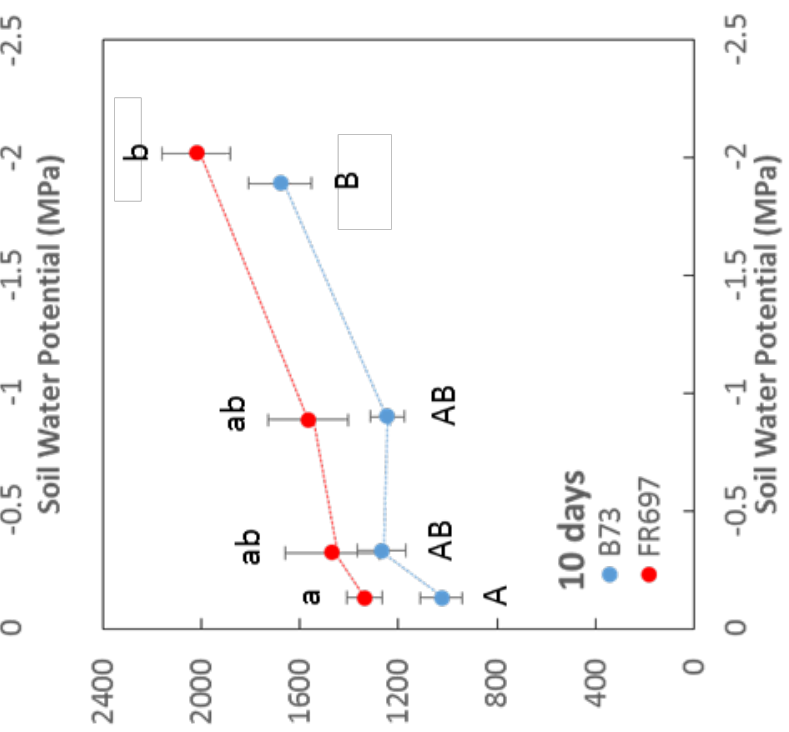

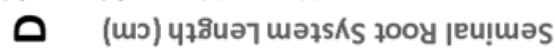

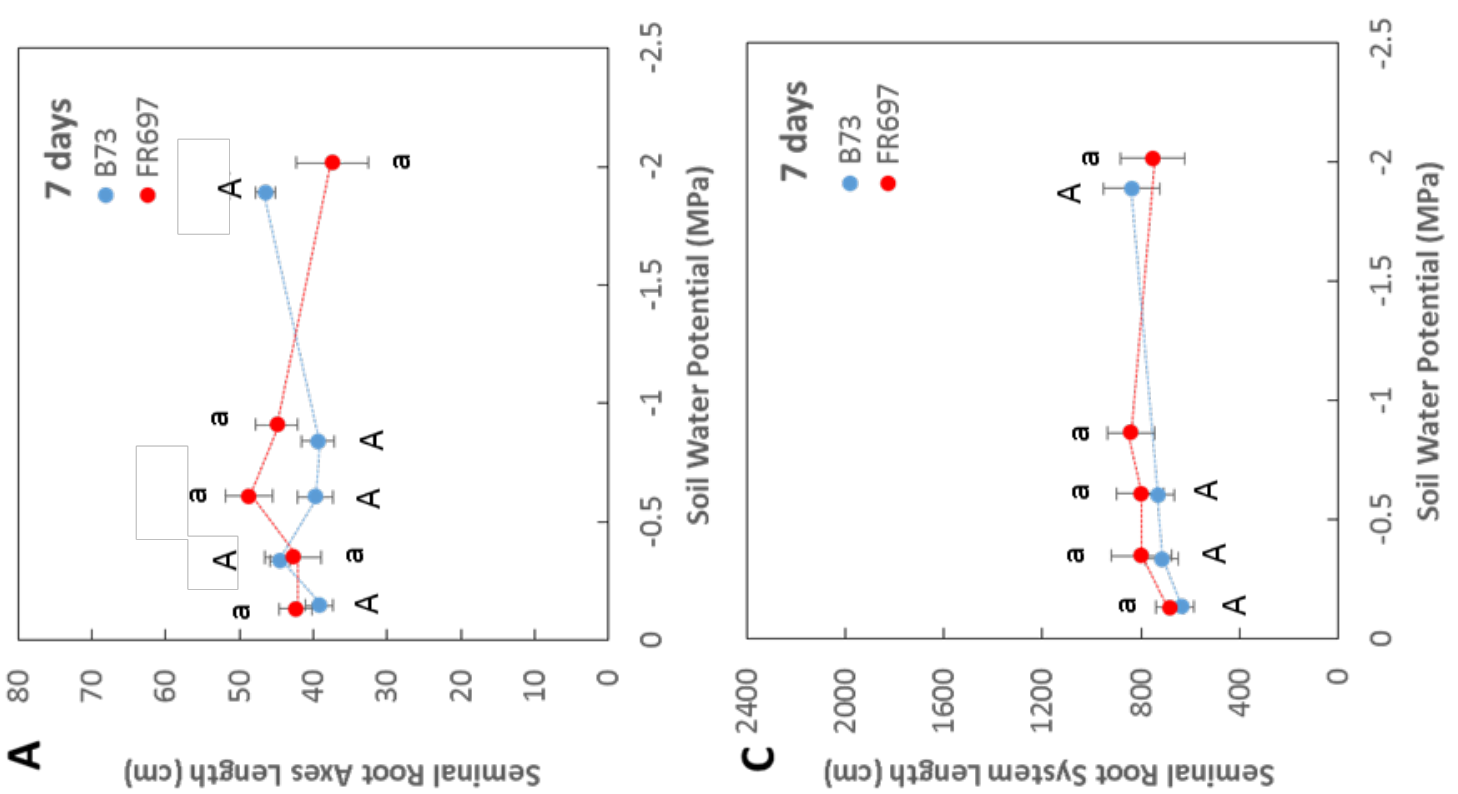


Figure 16. B73 and FR697 primary root axes length (A, B) and primary root system length, including lateral roots, (C, D) as a function of soil water potential in the outer chamber. Plants were grown for 7 days $(A, C)$ or 10 days $(B, D)$ in the outer chamber before harvest. Primary root axes length did not significantly increase under mild or severe water stress in either genotypes after 7 or 10 days of treatment. After 10 days of treatment with moderate or severe water stress (-0.9, 2.0 $\mathrm{MPa}$ ), the primary root system of FR697 proliferated significantly compared to well-watered plants, but growth increased only at severe stress in B73. Because the axes length did not change significantly, this increase in length was comprised of an increase in lateral root growth on the primary root axis. Water-stressed data are means \pm SE ( $n=5-12$ plants) from single or duplicate experiments. Wellwatered control data are means \pm SE $(n=12-22$ plants $)$ from three to four experiments. Within a genotype, means with different letters are significantly different, $p<0.025$. Means with the same letter are not significantly different, $p>0.05$. 


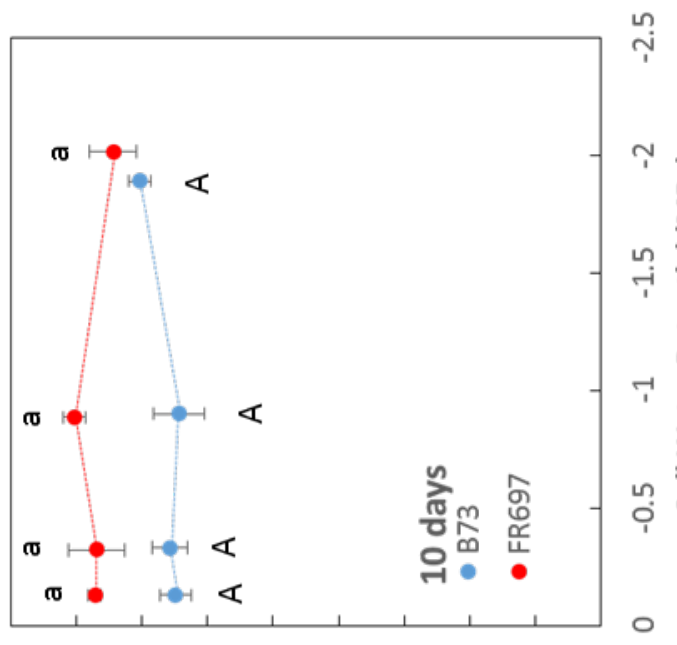

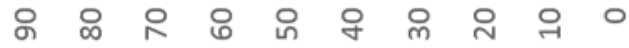

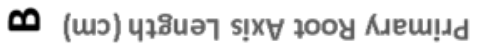

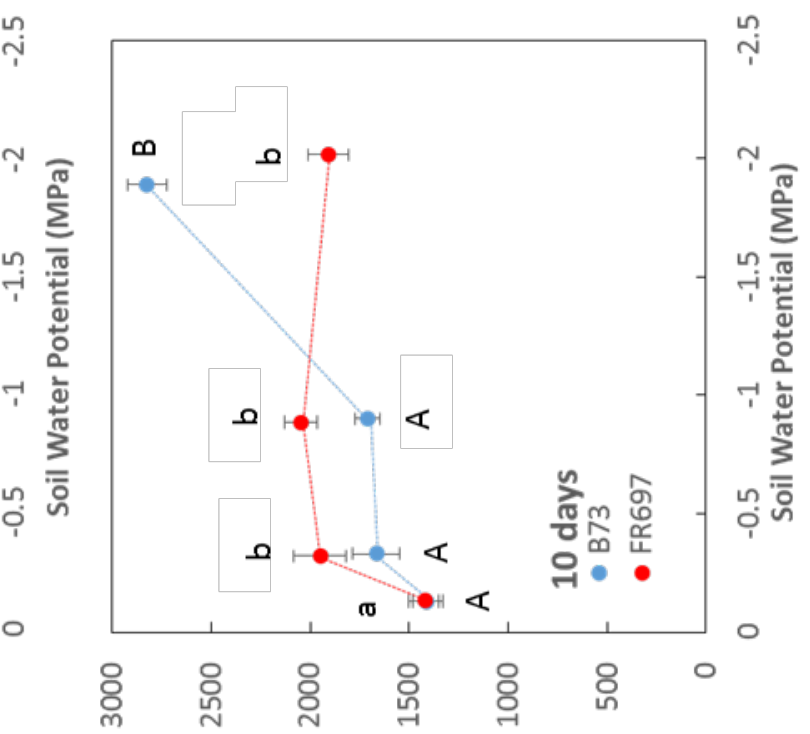

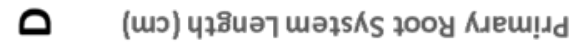

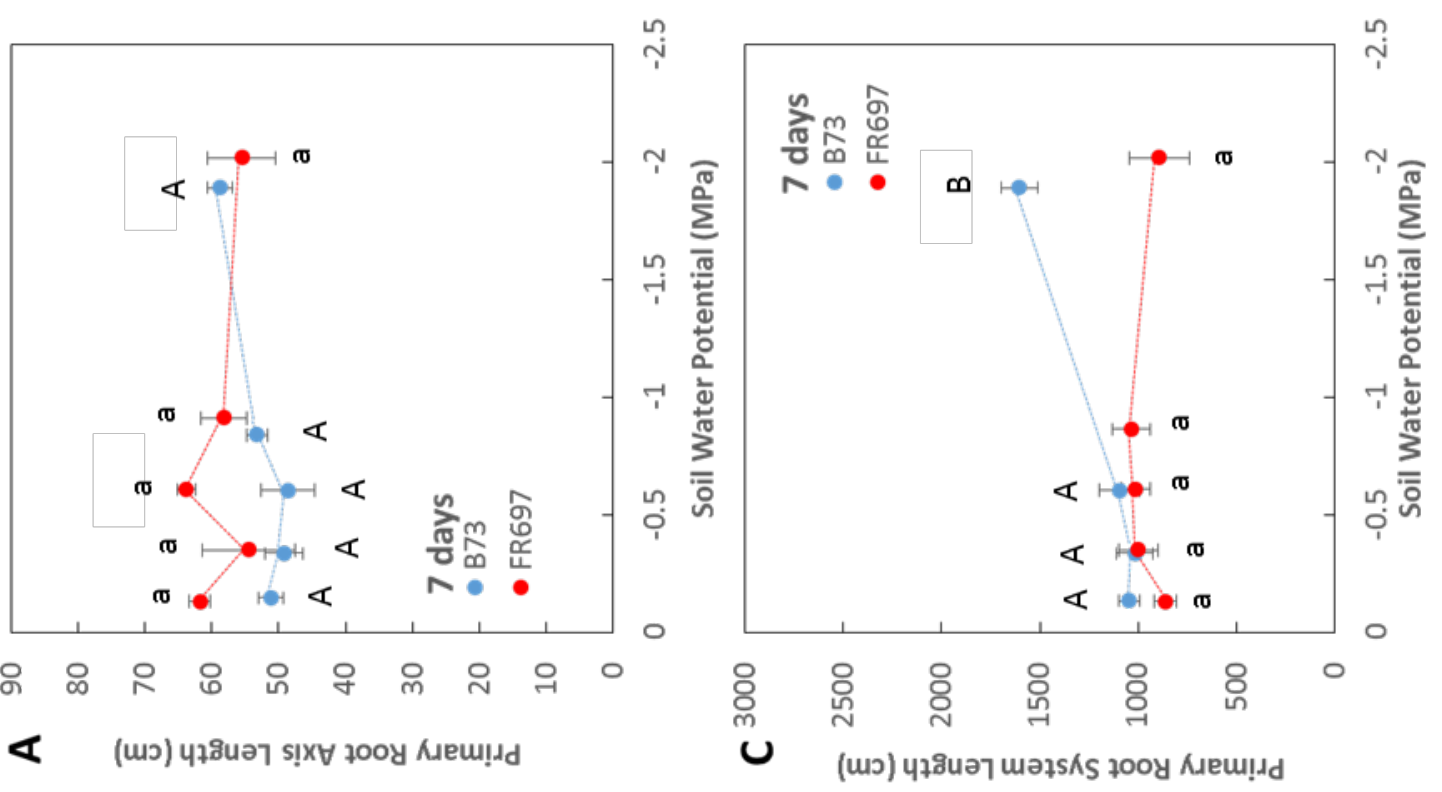




\section{Nodal lateral root growth decreases with low soil water potential in the outer chamber}

Node 1 lateral root length significantly decreased under mild and severe water stress conditions in both genotypes after 7 and 10 days of treatment (Fig. 17). Node 2 lateral root length also displayed a similar trend after 10 days of treatment. Node 2 lateral root length after 7 days of treatment did not show a significant difference in length over the range of soil water potentials. The laterals on these roots were not all initiated yet due to the young developmental age of the Node 2, 7 day axes. The number of nodal axes did not differ significantly from well-watered plants over the range of low soil water potentials after 7 and 10 days of treatment. B73 grown in -0.9 MPa soil for 7 days did have significantly decreased number of Node 1 axes, but this trend was not reproduced in plants treated for 10 days (Fig. 18).

\section{DISCUSSION:}

Upon building a new model system for the study of nodal root growth response to low soil water potentials, it was useful to characterize growth in the system over a range of water potentials. This was especially useful to uncover any unforeseen and interesting growth patterns. Growth responses of first and second order nodal roots from B73 and FR697 maize genotypes were characterized over a range of soil water potentials. As the growth responses of the nodal roots were characterized along with their root tip water potentials, exciting and unexpected results emerged from the data. 
Figure 17. B73 and FR697 lateral root system length of Node 1 (A, B) and Node $2(C, D)$ as a function of soil water potential in the outer chamber. Plants were grown for 7 days $(A, C)$ or 10 days $(B, D)$ in the outer chamber before harvest. Nodal lateral root length significantly decreased under mild or severe water stress in both genotypes after 7 or 10 days of treatment. Water-stressed data are means \pm SE ( $n=5$-11 plants) from single or duplicate experiments. Well-watered control data are means \pm SE $(n=12-17$ plants) from three to four experiments. Within a genotype, means with different letters are significantly different, $p<0.025$. Means with the same letter are not significantly different, $\mathrm{p}>0.05$. 

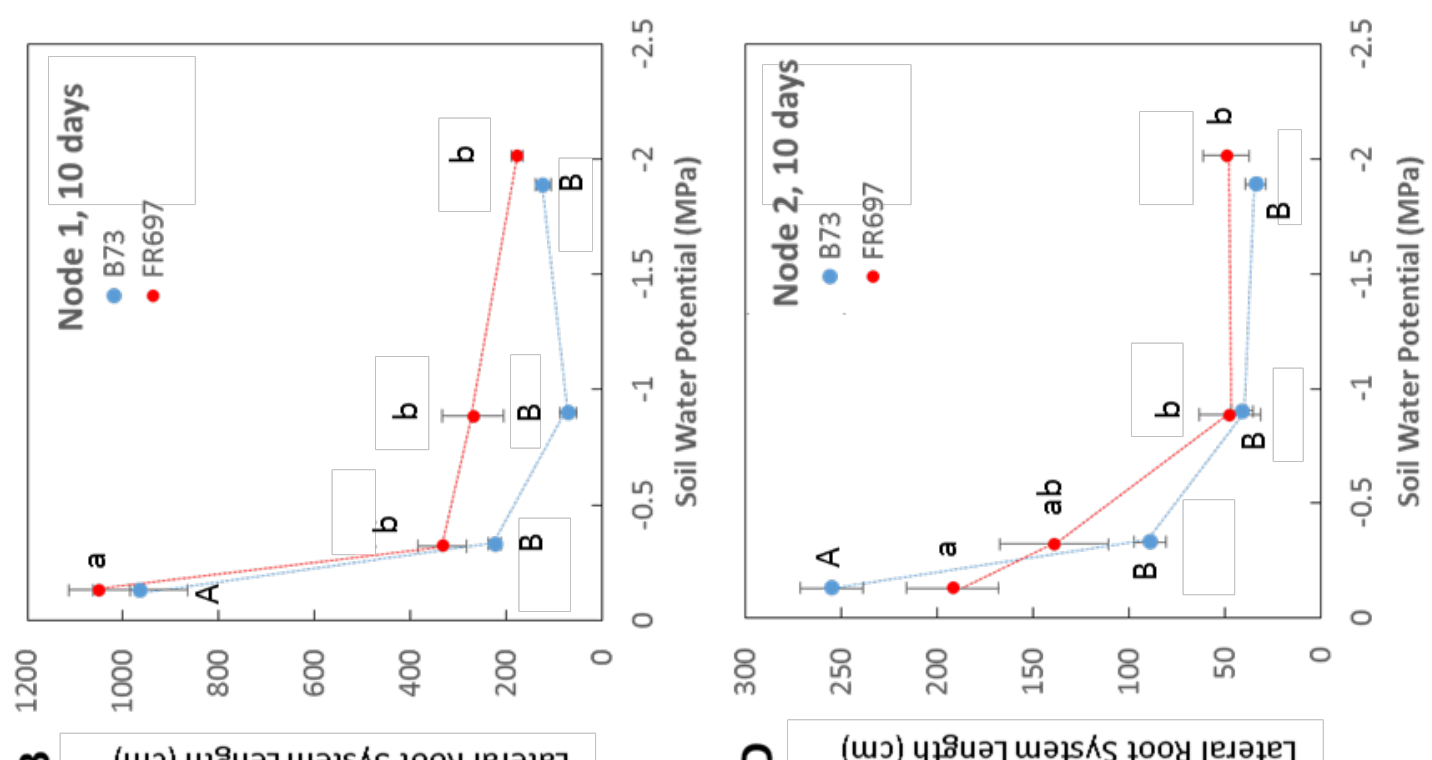

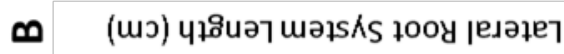
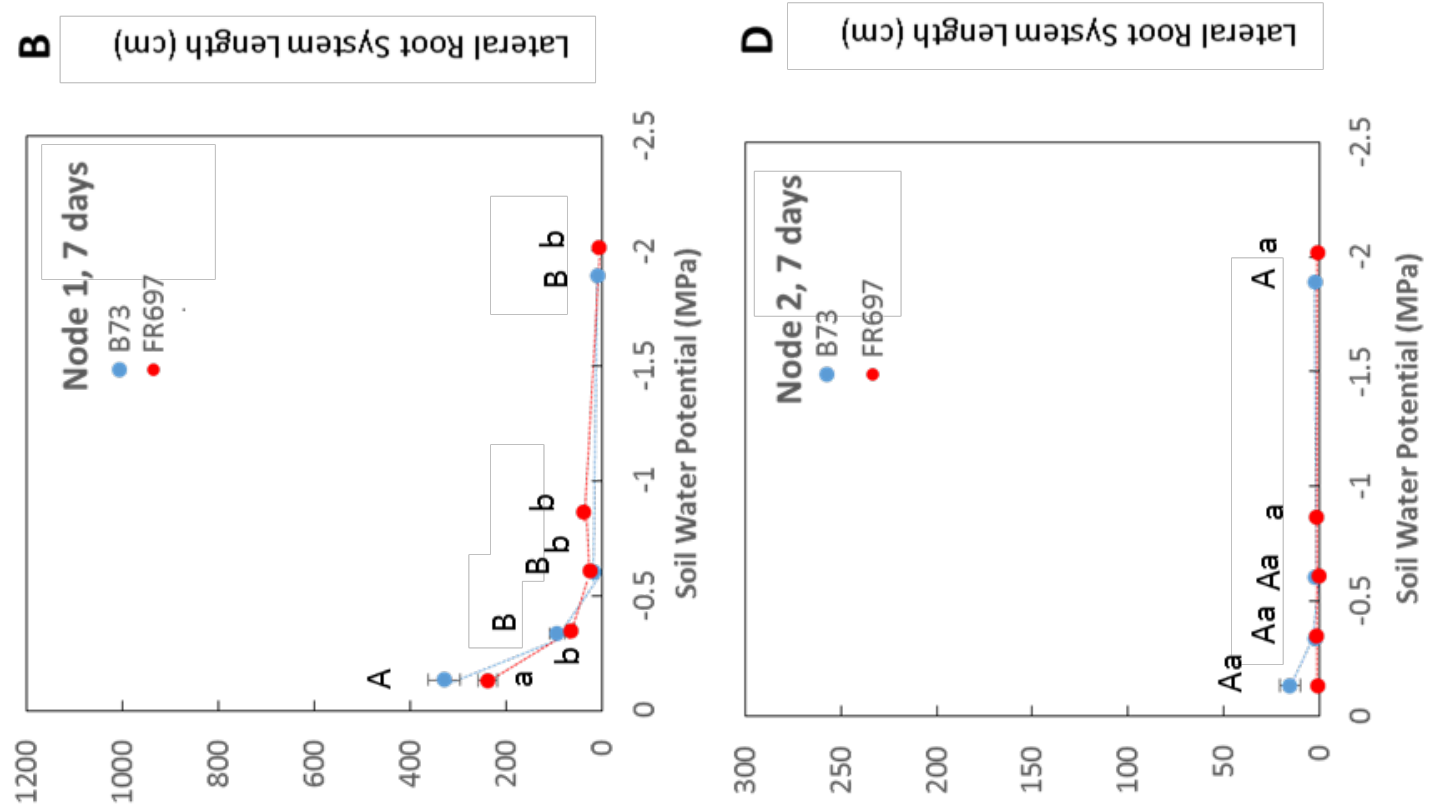

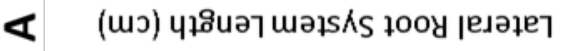

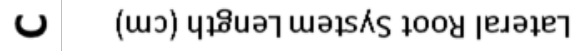


Figure 18. B73 and FR697 number of nodal root axes in Node $1(A, C)$ and Node $2(B, D)$ as a function of soil water potential in the outer chamber. Plants were grown for 7 days $(A, B)$ or 10 days $(C, D)$ in the outer chamber before harvest. Number of nodal axes did not differ significantly from well-watered plants over the range of water stress levels. B73 grown in moderate stress $(-0.9 \mathrm{MPa})$ for 7 days did decrease the number of Node 1 roots, but this trend was not reproduced in plants treated for 10 days. Water-stressed data are means \pm SE $(n=6-12$ plants $)$ from single or duplicate experiments. Well-watered control data are means $\pm \mathrm{SE}$ $(n=13-22$ plants) from three to four experiments. Within a genotype, means with different letters are significantly different, $p<0.025$. Means with the same letter are not significantly different, $\mathrm{p}>0.05$. 

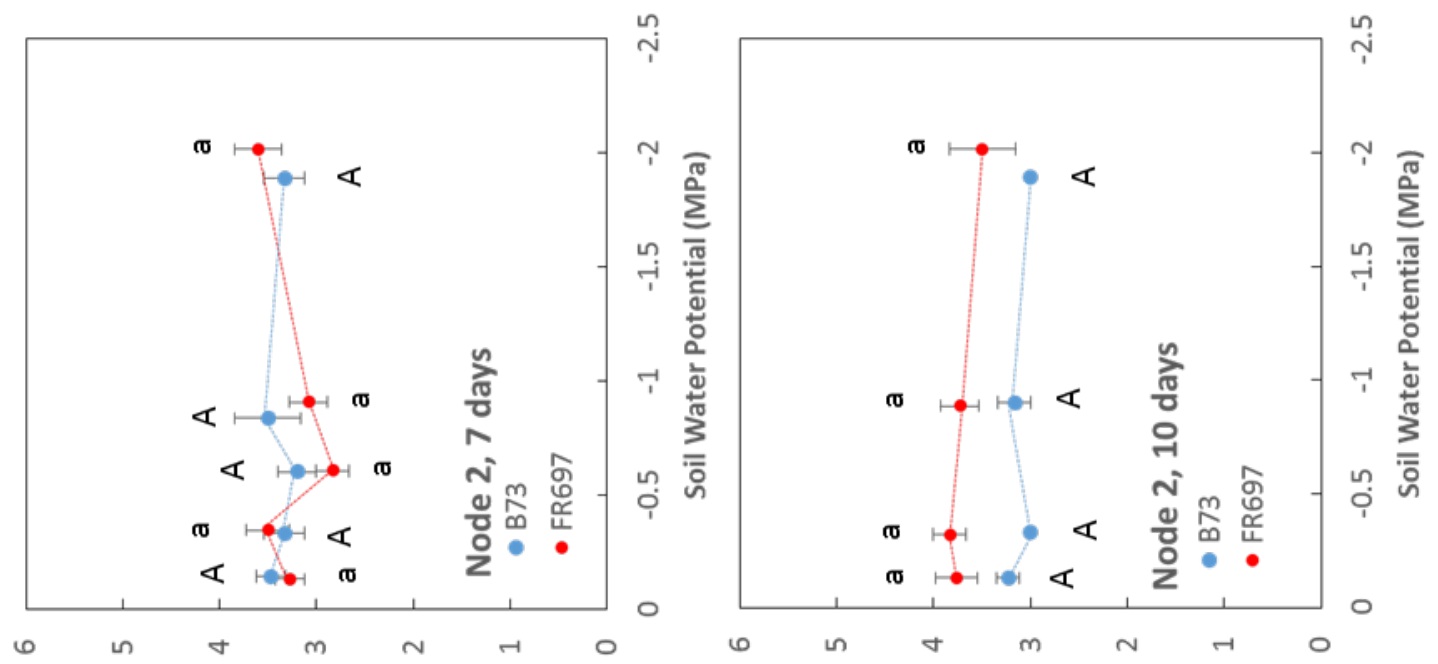

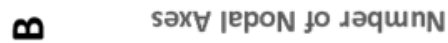

อ $\operatorname{sax\forall }$ IepoN to dəquinN

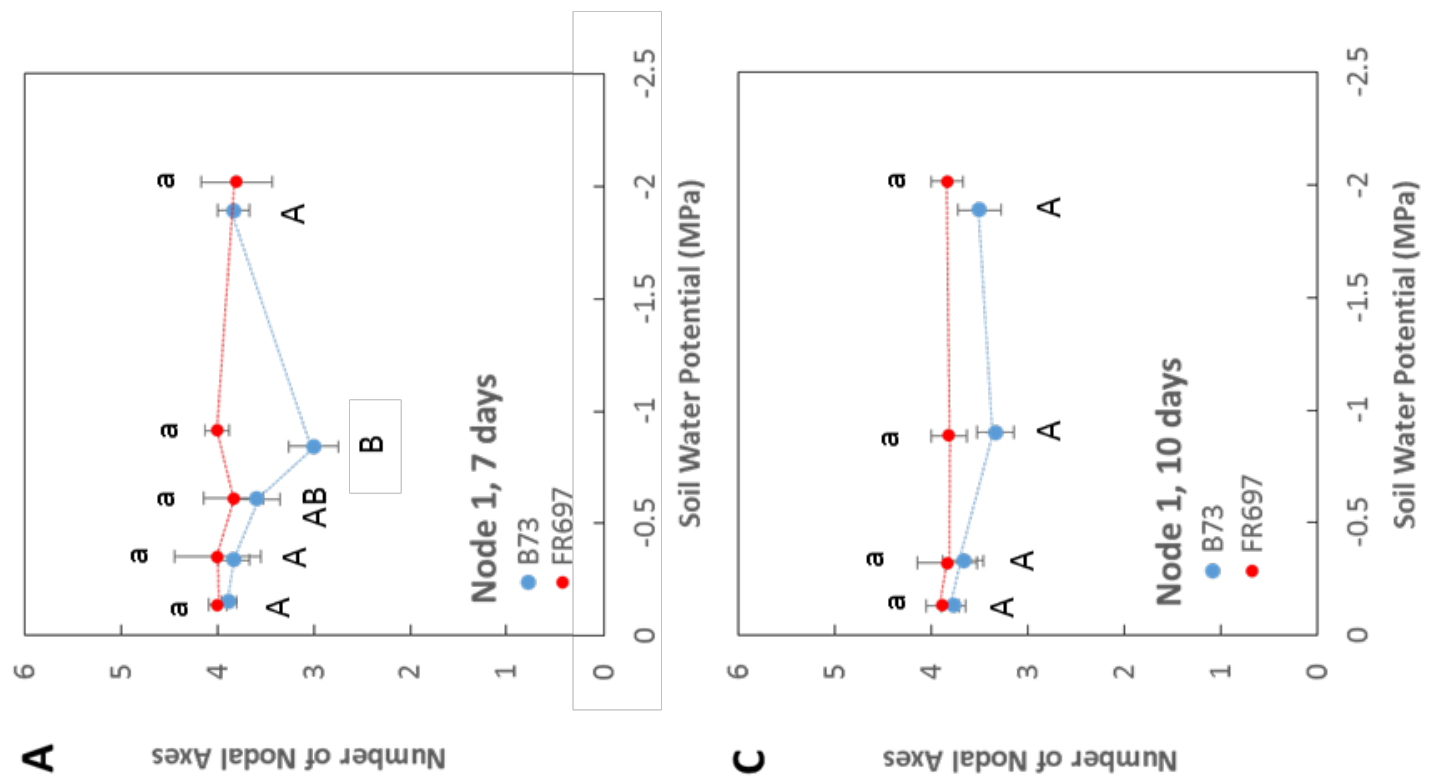


Maize nodal roots have been shown to maintain growth at low soil and tissue water potentials (Sharp and Davies, 1979; Westgate and Boyer, 1985), so it was hypothesized that nodal root growth would be maintained in mildly to moderately low soil water potentials. Data collected on the nodal axes root lengths and nodal root elongation rate from the divided-chamber system were not consistent with these previous findings (Figs. 1, 8, 9). It was discovered that the nodal axes length of both B73 and FR697 was sensitive to mildly low soil water potentials (Fig. 1).

The heightened sensitivity of nodal roots grown in the divided-chamber system may differ from previous studies for various reasons. First, the maize line that was used in previous studies was a hybrid (Westgate and Boyer, 1985), which may have exhibited a more vigorous hybrid growth unlike the inbred lines compared in the divided-chamber system. Secondly, the plants studied previously (Westgate and Boyer, 1985) were older than the young plants treated in the divided-chamber system. The nodal roots studied previously were at least from the third node. Based on the growth of Node 2 in comparison to Node 1 in this study, Node 3 may have an increased capacity to conduct water to the root tip than either of the nodes reported in the divided-chamber system.

\section{Hydraulic conductivity of nodal axes}

A surprising result of this study was the growth maintenance of the nodal root axes over the lowest range of soil water potentials (-0.9 MPa, $-2.0 \mathrm{MPa})$. 
Because the root tips were more hydrated than the soil (Fig. 4), water was conducted into the root tip from the plant through the root axis. Follow-up studies were conducted to measure differences in tissue water potential along the mature region of the root axes immediately following the growth zone (Figs. 10, $11,12)$.

The root axes water potentials indicated that FR697 had an increased capacity to conduct water into the root axes tissue or a decreased loss of water to the soil. The water potential of Node 1 B73 axes was much lower than FR697 Node 1 axes (Fig. 10A, 10C), suggesting poor conductivity of water along the axis to maintain root growth or an increase in leakage of water out of the axes into the surrounding soil. This was further illustrated by the increasing water stress in the tissue of B73 Node 1 as the roots continued to grow farther away from the hydrated shoot in the day 10 data (Fig. 12A).

The nodal roots harvested from the divided-chamber system showed growth induced water potentials, as the axes had higher water potentials than the water potentials in the growing regions (Figs. 10, 11, 12). This gradient was steeper in B73 than FR697 in both nodes, which indicated genotypic diversity in the increasing resistance to water movement into the growth zone (Fig. 10). Because the tissue immediately behind the tip (20 $\mathrm{mm}$ from apex) displayed a higher water potential, and the root tip was maintained at a much lower water potential, the water flow may have been hindered throughout the root tip.

The water potential of the cortical cells reflected the magnitude of water loss to the soil and the flux of water coming in from the vasculature. It served as 
an indication of how readily the water is transported from the stem into that region of the root. Because FR697 maintained a higher tissue water potential, it indicated FR697 may have had a higher hydraulic conductivity in the root. Because the degree to which the root was able to source water internally and maintain a higher water potential in the root tip directly impacted the growth of the root, the question must be asked what mechanisms are differentially regulated by the genotypes to facilitate water movement into and restrict water loss out of the root tips.

Wiegers et al. (2009) predicted that higher phloem flux rates would promote root growth under water stress. Inhibition of lateral root growth, which feeds off the transport phloem (De Schepper et al., 2013), may serve to increase the flux rates in the transport phloem under water stress to the apical root tip. Wiegers et al. also predicted roots may adapt to water stress by increasing the proximity of phloem elements to the growth zone to provide phloem water to the stressed root tip (Wiegers et al., 2009). Shortening of the growth zone in maize root tips has been shown under water stress (Sharp et al., 1988), and this likely does serve to shorten the distance between the meristematic and elongating cells in the root tip to the differentiated sieve tube elements. In addition, if root tips had shorter growth zones, the phloem elements would possibly be closer to the expanding non-suberized cells, which are susceptible to losing water to the soil (Brouwer, 1965).

Hydraulic lift through interconnected xylem elements may have played a significant role in mitigating the stress of the nodal root tips in the evenings when 
transpiration and xylem tension decreases (Caldwell et al., 1998). However, due to the low water conducting capacity of early metaxylem, the lack of late metaxylem, and the low stomatal resistance of young seedlings, the study posits a water limitation in young maize plants (Wentzel et al., 1989). It has been shown that the lack of xylem vessel elements near the tip imposes a high resistance to water flow in young maize roots (Wang et al., 1991). Kramer also predicted greater resistance to longitudinal water movement through the xylem in young tissues where xylem has not yet differentiated (Kramer, 1969). As the plant matures and develops an increasing system of late metaxylem, the water conducting capacity increases dramatically (Wenzel et al., 1989). These limits to hydraulic conductivity could help explain the unexpected sensitivity of Node 1 , the most immature nodal roots, to water stress in both genotypes (Fig. 1).

\section{Possible suberization response}

The mechanisms underlying this genotypic diversity may include the induction of a water-proofing system such as suberization or the formation of an exodermis. Portas and Taylor made a keen observation that if water and ions are lost through the "leaky" elongation zone, then enough water must be supplied to it to maintain turgor in the growing cells (Portas and Taylor, 1976). Brouwer also noted ions and water may leave the root more readily if the tissue has not been suberized. This may be an advantage of the shortening growth zone, to limit the leakiness of growing, un-suberized cell surface area (Brouwer, 1965). 
In Node 1, growth rate and root tip water potential continued to decrease in B73 whereas the water potential increased in FR697 with decreasing soil water potential (Fig. 4A). This suggests there may have been some response of the tissue to inhibit the release of water flowing out of the root to maintain the water potential of the root tip and growth rate despite the low soil water potential.

Because there was not much change in the water potential of the mature tissue between FR697 Node 2 at 7 and 10 days (Figs. 10D and 11), with the root axis at different lengths (Fig. 1B, 2A), this may further demonstrate how well connected the FR697 Node 2 roots are to the hydrated plant and how well the plant facilitates water transport to the tip, whether through vasculature connections, aquaporin regulation, or prevention of water loss from the root.

\section{Carbon partitioning and plasticity of the root system}

Due to the nature of the divided-chamber system, the total root system had access to soil water in parts of the root system other than the nodal roots, creating a plasticity response to heterogeneous soil conditions. Whereas previous studies imposed water stress through withholding water, this would affect the entire root system and not only nodal roots (Westgate and Boyer, 1985).

The importance of plasticity in the root system has been discussed extensively (Pregitzer et al., 1993). The ability of a root system to sense the availability of water, nutrients, and the path of least mechanical resistance 
promotes the most efficient use of carbon in the plant while also retrieving more resources for continued growth.

Although the water potential of the inner chamber did not change as part of the soil treatment, growth in the inner chamber varied as the nodal root system was treated with different soil water potentials (Figs. 15 and 16), indicating a repartitioning of carbon in the root system. Interestingly, although the seminal and primary root axes were not bound at the bottom of the pot, their axial length did not increase in response to low soil water potential (Figs. 15A, 15B, 16A, 16B). Instead, the seminal and primary lateral roots increased in response to water stress in the outer chamber (Figs. 15 and 16), and lateral roots have been shown to absorb a large amount of water (Ahmed et al., 2015).

It was not clear if the re-partitioning of carbon shown here is an active proliferation of the seminal and primary root system or a passive diffusion of photosynthates into other growing portions of the root system. Preliminary experiments in the lab suggested even with nodal roots excised, the seminal and primary root systems proliferate in the inner container (Tyler Dowd, University of Missouri, unpublished).

In previous split root studies, the growth of the nodal root system was also observed in water deficit conditions. Nodal root system growth in wheat was observed to increase under water deficit, but the growth response of the nodal root axes was not distinguished (Volkmar, 1997). The magnitude of the length increase, which included the entire nodal lateral root system, suggested that the length increase may have been due to an increase in the lateral root system. The 
lateral root system also proliferated at low soil water potentials in millet (Rostamza et al., 2013). Inconsistent with these results, in the present study the nodal lateral root system was significantly inhibited by low soil water potentials (Fig. 17). Unlike millet (Rostamza et al., 2013), the two maize lines studied here did not show a decrease in the number of nodal axes in response to low soil water potential for either node of roots (Fig. 18).

\section{Leaf growth in the divided-chamber system}

Maize leaf growth has been shown to be sensitive to water stress, decreasing as the leaf tissue water potential decreased (Westgate and Boyer, 1985). This growth response is thought to be a vital adaptation to drought, limiting the plant's leaf area to reduce the rate of water loss from the soil and prolonging the availability of water in the soil.

While previous studies looked at the growth inhibition of leaves and roots by withholding water, the divided-chamber system allowed the maintenance of leaf hydration and growth. Although water potential of the leaves was not measured, shoots growing in the divided-chamber system had access to water through the well-watered seminal and primary root systems. Therefore, the leaves may have been more hydrated and less inhibited in their growth pattern than if the shoot did not have access to water.

Photosynthesis is extremely sensitive to water stress (Boyer, 1970; Tezara et al., 1999). As the nodal roots perceived low soil water potentials, it is likely the transpiration rate and photosynthetic rate of the plant decreased due to 
signals originating from the roots (Zhang and Davies, 1991; Dodd, 2005). In the likely photosynthate-limited plant, maintained leaf growth under mildly low soil water potentials could have become a competing sink with nodal root growth. While nodal root growth was most sensitive at $-0.3 \mathrm{MPa}$ soil treatment, leaf growth was maintained (Figs. 1 and 13). This result is not consistent with the sensitive growth pattern of leaves and the resilient growth pattern of nodal roots in previous studies (Westgate and Boyer, 1985; Volkmar 1997; Rostamza et al., 2013). The inner chamber supplying water to the shoot may be a possible explanation.

While water stress was not imposed on the leaves, leaf growth was inhibited when the nodal root system was treated with -2.0 MPa soil. It has been shown that roots have the ability to signal to the shoots, inhibiting growth under water deficit conditions (Dodd 2003; Munns and Cramer, 1996). The growth inhibition of the leaves at -2.0 MPa may have been affected by such signals from the nodal roots. Alternatively, leaf water potential could have dropped under this extreme water deficit condition, limiting the growth of the leaf tissue. The leaf growth inhibition at -2.0 MPa soil treatment may have enabled the plant to repartition the carbon to maintain nodal root growth under severe water deficit. 


\section{CHAPTER 4}

Future studies and implications for translational research 


\section{Introduction}

The divided-chamber system provides a powerful experimental approach to investigate the physiological mechanisms regulating nodal root growth responses to adverse soil conditions. The divided-chamber system is currently functional in testing differences in hydraulic conductivity inside the root system and plasticity of growth.

In the present study it was discovered that the two contrasting genotypes may not be very different physiologically in their nodal root growth response to low water potentials. Although this study began as an investigation of the differential abilities of two contrasting genotypes to maintain growth under water stress, very little can be said about their physiological differences under water stress through these results because the nodal roots of FR697 were not comparatively stressed relative to $\mathrm{B} 73$. There was a large difference in the ability of the genotypes to facilitate the conductance of water into the root tip in order to maintain a high root tip water potential and sustain growth (see Chapter 3).

Unlike previous split root studies, the water potential of the growing root tip was measured to investigate the possible effect of root tip water potential maintenance on the growth response. Because the plant had access to water through the inner chamber, hydraulic conductivity was thought to be an important influence on root growth through dry soil. It was previously demonstrated that roots have the ability to maintain growth through air, based purely on phloem water sources (Boyer et al., 2010). The present study highlights the importance 
of a plant's ability to maintain higher root tip water potentials under water stress conditions.

Also unique to this divided-chamber system, the study characterized the growth of nodal roots in respect to their developmental node. Because of the design of the precise and highly-controlled divided-chamber system, it was possible to study each node independently and to delineate the responses of

distinct nodes of roots. Because the growth patterns were reproducible, this also limited the variability between samples and produced a more robust analysis of growth patterns in response to precise low soil water potentials. Because of its reproducibility, the divided-chamber system could be used to study detailed mechanistic questions regarding the maintenance of nodal root growth under water deficit conditions.

\section{Future anatomical studies}

The primary root in the maize seedling system has a shortened growth zone at -1.6 MPa relative to well-watered conditions (Yamaguchi and Sharp, 2010). It is expected that the growth zone is also shortened in nodal roots in response to low soil water potential, and this may serve to shorten the distance between the meristematic and elongating cells in the root tip to the differentiated sieve tube elements (Wiegers et al., 2009). The shortening of the growth zone may be analyzed in future studies along with the proximal distance from the root apical meristem to the closest differentiated phloem and vessel element. 
Because water flows more readily through vascular elements, future anatomical characterizations of root tips and axis tissue would be informative about the hydraulics of the root axis and root tip. It has been shown that the lack of maize vessel elements near the tip imposes a high resistance to water flow in young roots (Wang et al., 1991). Over an extended period of time, the plants will morphologically adapt to the dry environment. It is expected that the width of vessel elements would increase and the number of elements would increase with severity of water stress. FR697 is expected to have larger vascular elements than B73 due to its superior ability to maintain high tissue water potentials. Node 2 is expected to have larger vascular elements than Node 1 due to its superior ability to maintain high tissue water potentials.

\section{Future hydraulic conductivity studies}

Future studies could help determine if the hydraulic conductivity of the nodal root axis, as measured with a root pressure probe (Bramley et al., 2007; Hose et al., 2000; Steudle et al., 1987), and the root tip, as measured with a cellular pressure probe (Hose et al., 2000), increase during water stress. Studies could also determine if these changes are genetically diverse or vary developmentally with the severity of water stress.

Whole root hydraulic conductivity measurements cannot measure the hydraulic conductivity of the root tip, which is the region of most interest in terms of water limitations to growth. Considering these limitations, hydraulic conductivity of cells in the root tip could be measured with a cellular pressure 
probe as described in (Hose et al., 2000). Due to the differences in aquaporin expression and abundance throughout the circadian cycle, the hydraulic conductivity of the roots may be measured at the end of the day and at the end of the night cycle. Hydraulic conductivity is expected to increase during the day in both genotypes, when PIP proteins are highly expressed relative to their expression in the dark (Hachez et al., 2012).

Hydraulic conductivity of the nodal root axis is expected to increase under water stress. FR697 is expected to have a greater hydraulic conductivity than B73 under water stress, due to its superior ability to maintain high water potentials under similar severe water stress conditions. Node 2 roots are also expected to have a greater hydraulic conductivity than Node 1 roots under water stress, due to their ability to maintain high root tip water potentials under similar low soil water potentials.

\section{Future aquaporin studies}

While maize aquaporin expression, protein content, and post-translational modification were not measured in this study, it would be an interesting next step to measure these in the root tips of B73 Node 2 and FR697 Node 2 under severe stress (-2.0 MPa). Because FR697 maintained a higher root tip water potential than B73 under severe stress, it is possible its aquaporin content is significantly higher. This would also help separate differences in hydraulic conductivity due to anatomical differences, such as number, width, and proximity of sieve tube elements to the growing root tip. 
Transcripts from root tips could be purified and sequenced using RNAseq to identify known and probable aquaporin sequences and quantify their relative abundance. Level of protein detection in water-stressed samples could be compared to well-watered samples of the same genotype and of the same node.

Aquaporin expression and aquaporin protein abundance are expected to increase under water stress, and it is also expected to increase more in FR697 relative to B73 due to its superior ability to maintain high root tip water potentials under water stress. Aquaporin expression and protein abundance are also expected to increase more in Node 2 relative to Node 1 due to its superior ability to maintain high tissue water potentials under water stress. Differences in aquaporin homologs are expected between the two lines, which may explain differences in regulation of water status.

Plasma-membrane proteins could be purified and enriched using the protocol described for maize primary roots (Zhang et al., 2013). Level of protein detection in water-stressed samples could be compared to well-watered samples of the same genotype and of the same node. The aquaporins ZmTIP2-1 and ZmPIP1-2 would be good candidates for targeted protein abundance studies due to their significant abundance in the regions of the FR697 primary root where growth is maintained (Voothuluru et al., 2016). While the divided-chamber system has already displayed the importance of defining root types, that even nodes differ from each other in hydraulic conductance capabilities, there may be some redundancy of protein abundance within the apical root meristems in the same genotype. 


\section{Future studies with ABA}

The following future studies could help elucidate whether the stress level of the root tip is affected by ABA's positive influence on root hydraulic conductivity through aquaporins. The effects of ABA on hydraulic conductivity are unique to the type of tissue in which ABA is active (Dodd, 2013). While ABA decreases hydraulic conductivity in the shoot by inducing stomatal closure, creating greater resistance at the stomate level, $A B A$ is thought to decrease resistance to water movement in root cortical cells through effects on aquaporin content (Parent et al., 2009).

One commonly overlooked aspect of hormone experiments is the importance of the water stress level of the tissue, which may affect the plant's perception of endogenous hormone levels (Sharp, 2002; Tardieu and Davies, 1992). Therefore, while it would be meaningful to study the effects of $A B A$ while the plant is stressed, the exogenous application of ABA on a well-watered plant would be less meaningful because of the differential sensitivity of the tissues.

The split root system is amenable to studies of exogenous hormone treatment on the nodal roots. Not only are the roots visible against the face, allowing for immediate localization of the roots without disturbing their structure in the soil, but the transparent walls are also thin enough to cut and access intact roots of growing plants. After cutting a small flap in the transparency surrounding the root, exogenous ABA could be applied to the root tips of B73 and FR697 water stressed plants in physiologically relevant amounts (similar to those cited in Spollen et al. 2000). After treatments are applied, the cut transparency flap could 
be taped shut to prevent water loss from the soil and artifactual dehydration of the root tip.

Iso-osmotic solutions relative to the soil water potential would be applied to control plants. The water potential of ABA treatments will also be adjusted to the soil water potential so that the resulting tissue water potential cannot be attributed to an artifactual hydration of the tissue from the applied solution.

Application of exogenous ABA to water stressed nodal roots is expected to increase the hydraulic conductivity of the root tip by increasing aquaporin expression and aquaporin protein content (Parent et al. 2009). Previous studies with a pressure probe showed an increase in maize root hydraulic conductivity when the tissue was treated with exogenous $A B A$, acting presumably through aquaporins (Hose et al., 2000). This increase in hydraulic conductivity is expected to increase the tissue water potential of the root tip under water stress by partially alleviating resistance of water flow to the growing tip. Because B73 has lower endogenous levels of ABA under stress (Leach et al. 2011), exogenous ABA may have a more pronounced effect on water status than in FR697 with higher endogenous levels of ABA under stress (Leach et al. 2011).

\section{Future suberization studies}

Future studies may also include histological measurement or chemical extraction of suberin content in nodal roots under water deficit. Because FR697 showed a superior ability to maintain a high root tip water potential at a greater length from the stem, despite decreasing soil water potentials, it is possible this 
line had a greater suberization response to reduce the amount of water lost to the dry soil. It has been shown that water-stressed roots increase suberin content in the root to retain water (Schreiber et al., 2004; Steudle, 2000). The time to equilibration in the psychrometer cups for mature tissue relative to root tips increased (data not shown), and a similar increase in equilibration time was seen in water stressed tissue relative to well-watered tissue measurements. This observation suggested that water movement out of the root tissue was negatively affected and may have indicated the presence of suberization.

\section{From the lab to the field}

There is a need for meaningful, translational results from the lab to the field. With a greater emphasis on sustainable intensification, we are learning to create more food with fewer resources. This intensification necessitates the transition of knowledge discovered in the lab to implementation and candidate testing in the field to improve the crop varieties available to farmers.

There are many artifacts inherent to studies in lab that pose difficulties in translating findings to the field. Efforts must focus on key parameters to interpret the results and better ascertain those improvements that will most effectively increase plant growth and survival in unfavorable, rapidly changing climatic conditions. Tissue water potential is integral to the accurate interpretation of physiological studies in both the lab and the field. Without measuring the soil or tissue water potential, experiments and results are difficult to reproduce and compare. 
Future studies in the field may be able to study the growth of older nodes of roots, which are difficult to study in the lab due to the size of the pot required for unrestricted growth of the seminal, primary, and Node 1 root axes, while studying Node 3. Because Node 2 is more resilient than Node 1 of B73 and FR697 in maintaining a high root tip water potential, even higher rates of hydraulic conductivity may be seen in Node 3 . Mechanisms enabling the increased hydraulic conductivity may be elucidated and selected for in breeding programs to enable the growth of deeper rooting, more resilient maize varieties. 


\section{APPENDIX 1: ANATOMICAL STUDIES}


An anatomical study of nodal root tips was performed to measure the nodal root growth zones and the proximity of the nearest sieve tube elements of the different genotypes in well-watered and severe water deficit conditions (approximately -2.0 MPa). Cross sections were analyzed for morphological changes in the number and width of vessel and phloem elements in the root tip, particularly those that varied between genotypes and stress levels.

Nodal root tips that were used in root tip water potential measurements were fixed in FAA (formalin, acetic acid, alcohol) and processed by Indexx BioResearch Labs. Root tips were dehydrated through an ethanol dilution series, then embedded in paraffin. Parrafin blocks were sectioned with a microtome into $4 \mu \mathrm{m}$ sections and stained with a combination of alcian blue and safranin- $\mathrm{O}$ (Tolivia and Tolivia, 1987). This combination of dyes was selected to stain the targets lignin, suberin, and cutin (red) and cellulose (blue). It was a highly reproducible stain and reduced non-specific binding of safranin (Ruzin, 1999; Tolivia \& Tolivia, 1987). A wide-field light microscope, Zeiss Axiovert 200M, was used to image samples at the University of Missouri Molecular Cytology Core Facility.

The anatomical study was inconclusive due to poor tissue embedding. Several layers of the embedded wax roots were cut from the paraffin blocks, but all layers of the tissue were deformed. The tissue embedding process may have been impeded by petroleum coating the roots, artifactual remnants from the psychrometer measurements of root tip water potential. If the petroleum coated the tissue, it may have stopped the root from properly dehydrating through the 
ethanol series and filling with paraffin. Because it was impossible to determine the degree of shrinkage the cells had undergone, the samples were not analyzed. 


\section{REFERENCES}

Ahmed M, Zarebanadkouki M, Kaestner A, Carminati A. 2015. Meaurements of water uptake of maize roots: the key function of lateral roots. Plant and Soil. 398:1-19.

Bagley JE, Miller J, Bernacchi CJ. 2015. Biophysical impacts of climate-smart agriculture in the midwest United States. Plant, Cell and Environment 38 (9):1913-1930.

BaluškaF, Mancuso S, Volkmann D, Barlow PW. 2004. Root apices as plant command centres: the unique 'brain-like' status of the root apex transition zone. Biológia (Bratislava) 59 (13):7-19.

Bolaños J, Edmeades G 1996. The importance of the anthesis-silking interval in breeding for drought tolerance in tropical maize. Field Crops Research 48, 65-80.

Boyer J.S., Byrne P., Cassman K.G., Cooper M., Delmer D., Greene T., Gaffney J. 2013. The US drought of 2012 in perspective: a call to action. Global Food Security 2, 139-143.

Boyer JS, Silk WK, Watt M. 2010. Functional Plant Biology 37: 1105-1116.

Boyer JS. 1982. Plant productivity and the environment. Science. 218: 443-448.

Boyer JS. 1970. Leaf enlargement and metabolic rates in corn, soybean, and sunflower at various leaf water potentials. Plant Physiology 46:233-235. 
Boyer JS, Knipling EB. 1965. Isopiestic technique for measuring leaf water potentials with a thermocouple psychrometer. Proceedings of the National Academy of Sciences of the United States of America 54, 1044-1051.

Bramley H, Turner NC, Turner DW, Tyerman SD. 2007. Comparison between gradient-dependent hydraulic conductivities of roots using the root pressure probe: the role of pressure propagations and implications for the relative roles of parallel radial pathways. Plant, Cell and Environment 30(7): 861874.

Brouwer R. 1965. Ion absorption and transport in plants. Annual Review of Plant Physiology 16:241-266.

Caldwell MM, Dawson TE, Richards JH. 1998. Hydraulic lift: consequences of water efflux from the roots of plants. Oecologia 113: 151-161.

Canny MJ and Huang CX. 1994. Rates of diffusion into roots of maize. New Phytologist 126: 11-19.

Chaumont F and Tyerman SD. 2014. Aquaporins: Highly Regulated Channels Controlling Plant Water Relations. Plant Physiology 164: 1600-1618.

Chaves MM, Maroco JP, Pereira JS. 2003. Understanding plant responses to drought - from genes to the whole plant. Functional Plant Biology 30:239264. 
Davies WJ, Zhang J, Yang J, Dodd IC. 2011. Novel crop science to improve yield and resource use efficiency in water-limited agriculture. Journal of Agricultural Science 149:123-31.

De Schepper V, De Swaef T, Bauweraerts I, Steppe K. 2013. Phloem Transport: A Review of Mechanisms and Controls. Journal of Experimental Botany 64 (16), 4839-4850.

Dembinsky D, Woll K, Saleem M, Liu Y, Fu Y, Borsuk LA, Lamkemeyer T, Fladerer C, Madlung J, Barbazuk B, et al. 2007. Transcriptomic and Proteomic Analyses of Pericycle Cells of the Maize Primary Root. Plant Physiology 145: 575-588.

Dodd IC. 2013. Abscisic acid and stomatal closure: a hydraulic conductance conundrum? New Phytologist 197: 6-8.

Dodd IC. 2005. Root-to-shoot signalling: assessing the roles of 'up' in the up and down world of long-distance signalling in planta. Plant and Soil 74:257275.

Dodd IC. 2003. Hormonal interactions and stomatal responses. Journal of Plant Growth Regulation 22:32-46.

Du T, Kang S, Zhang J, Davies WJ. 2015. Deficit irrigation and sustainable water-resource strategies in agriculture for China's food security. Journal of Experimental Botany 66(8):2253-2269. 
Elmore R, Abendroth L. 2007. Rootless corn syndrome. Integrated Crop Management News. Paper 1083.

Eshel A. and Beeckman T. 2013. Plant Roots: The Hidden Half, 4th edn Boca Raton, FL, USA: CRC press.

Fusseder, A. 1987. The longevity and activity of primary root of maize. Plant and Soil 101:257-265.

Gao Y, Lynch JP. 2016. Reduced crown root number improves water acquisition under water deficit stress in maize (Zea mays L.). Journal of Experimental Botany 67: 4533-4546.

Grubbs FE. 1950. Sample criteria for testing outlying observations. Annals of Mathematical Statistics 21 (1): 27-58.

Hachez C, Veselov D, Ye Q, Reinhardt H, Knipfer T, Fricke W, Chaumont F. 2012. Short-term control of maize cell and root water permeability through plasma membrane aquaporin isoforms. Plant, Cell and Environment 35: 185-198.

Harmon ME, Lajtha K. 1999. Analysis of detritus and organic horizons for mineral and organic constituents. In: Robertson GP, Bledsoe CS, Coleman DC, Sollins P (Eds.) Standard soil methods for long-term ecological research. Oxford University Press, New York, pp. 143-165.

Hochholdinger F. 2016. Untapping root system architecture for crop improvement. Journal of Experimental Botany 67(15): 4431-4433. 
Hochholdinger F Tuberosa R. 2009. Genetic and genomic dissection of maize root development and architecture. Current Opinion in Plant Biology 12: $172-177$.

Hochholdinger F, Woll K, Sauer M, Dembinsky D. 2004. Genetic dissection of root formation in maize (Zea mays) reveals root type-specific developmental programmes. Annals of Botany 93: 359-368.

Hodge A. 2004. The plastic plant: root reponses to heterogeneous supplies of nutrients. New Phytologist 162: 9-24.

Hoppe DC, McCully ME, Wenzel CL. 1986. The nodal roots of Zea: their development in relation to structural features of the stem. Canadian Journal of Botany 64: 2524-2537.

Horchani F, Aschi-Smiti S. 2010. Prolonged root hypoxia effects on enzymes involved in nitrogen assimilation pathway in tomato plants. Plant Signaling and Behavior 12:1583-1589.

Hose E, Steudle E, Hartung W. 2000. Abscisic acid and hydraulic conductivity of maize roots: a study using cell- and root-pressure probes. Planta 211: 874882.

Javot $\mathrm{H}$ and Maurel C. 2002. The Role of Aquaporins in Root Water Uptake. Annals of Botany 90: 1-13. 
Kang SZ, Zhang JH. 2004. Controlled alternate partial rootzone irrigation: its physiological consequences and impact on water use efficiency. Journal of Experimental Botany 55(407):2437-2446.

Kramer PJ. 1969. Plant and soil water relationships: A modern synthesis. New York: McGraw-Hill Book Co.

Lawlor DW, Cornic G. 2002. Photosynthetic carbon assimilation and associated metabolism in relation to water deficits in higher plants. Plant, Cell and Environment 25: 275-294.

Leach KA, Hejlek LG, Hearne LB, Nguyen HT, Sharp RE, Davis GL. 2011. Primary Root Elongation Rate and Abscisic Acid Levels of Maize in Response to Water Stress. Crop Science 51: 157-172.

Lilley JM Kirkegaard JA . 2011. Benefits of increased soil exploration by wheat roots. Field Crops Research 122, 118-130.

Lobell DB, Roberts MJ, Schlenker W, Braun N, Little BB, Rejesus RM, Hammer GL. 2014. Greater sensitivity to drought accompanies maize yield increase in the U.S. Midwest. Science 344:516-519.

Lynch J. P. 2013. Steep, cheap and deep: an ideotype to optimize water and N acquisition by maize root systems. Annals of Botany 112 347-357.

McLaughlin JE, Boyer JS. 2004. Sugar-responsive gene expression, invertase activity, and senescence in aborting maize ovaries at low water potentials. Annals of Botany 94: 675-689. 
McMullen MD, Kresovich S, Villeda HS, Bradbury P, Li H, et al. 2009. Genetic properties of the maize nested association mapping population. Science 325: $737-40$.

Miedema P, Post J, Groot P. 1987. The effects of low temperature on seedling growth in maize genotypes. In Agricultural Research Reports, Vol 926. Pudoc, Wageningen, The Netherlands, p 124.

Munns R, Cramer G. 1996. Is coordination of leaf and root growth mediated by abscisic acid? Plant and Soil 185:33-49.

Neales TF, Incoll LD. 1968. The control of leaf photosynthesis rate by the level of assimilate concentration in the leaf: a review of hypotheses. Botanical Reviews 34: 107-125.

Nonami H, Boyer JS. 1989. Turgor and growth at low-water potentials. Plant Physiology 89: 798-804.

Parent B, Hachez C, Redondo E, Simonneau T, Chaumont F, Tardieu F. 2009. Drought and abscisic acid effects on aquaporin content translate into changes in hydraulic conductivity and leaf growth rate: a transscale approach. Plant Physiology 149: 2000-2012.

Passioura J. 2006. The perils of pot experiments. Functional Plant Biology 33(12): 1075-1079.

Portas C, Taylor H. 1976. Growth and survival of young plant roots in dry soil. Soil Science 121:170-175. 
Pregitzer, K.S., R.L. Hendrick, and R. Fogel. 1993. The demography of fine roots in response to patches of water and nitrogen. New Phytologist 125: 575580.

Pritchard J, Fricke W, Tomos D. 1996. Turgor-regulation during extension growth and osmotic stress of maize roots. An example of single cell mapping. Plant and Soil 187: 11-21.

Ribaut JM, Pilet PE. 1994. Water stress and indol-3yl-acetic acid content of maize roots. Planta 193: 502-507.

Rostamza M, Richards RA, Watt M. 2013. Response of millet and sorghum to a varying water supply around the primary and nodal roots. Annals of Botany 112: $439-446$.

Saab I.N., Sharp R.E., Pritchard J. 1992. Effect of inhibition of abscisic acid accumulation on the spatial distribution of elongation in the primary root and mesocotyl of maize at low water potentials. Plant Physiology 99: 2633.

Saab I.M., Sharp R.E., Pritchard J., Voetberg G.S. 1990. Increased endogenous abscisic acid maintains primary root growth and inhibits shoot growth of maize seedlings at low water potentials. Plant Physiology 93: 1329-1336.

Schnable PS et al. 2009. The B73 maize genome: complexity, diversity, and dynamics. Science 326: 1112-1115. 
Schreiber L, Franke R, Hartmann K. 2004. Wax and suberin development of native and wound periderm of potato (Solanum tuberosum L.) and its relation to peridermal transpiration. Planta 220: 520-530.

Shapiro SS and Wilk MB. 1965. An analysis of variance test for normality (complete samples). Biometrika 52 (3-4): 591-611.

Sharp RE, PoroykoV, Hejlek LG, Spollen WG, Springer GK, Bohnert HJ, Nguyen HT. 2004. Root growth maintenance during water deficits: physiology to functional genomics. Journal of Experimental Botany 55: 2343-2351.

Sharp RE. 2002. Interaction with ethylene: changing views on the role of ABA in root and shoot growth responses to water stress. Plant, Cell and Environment 25: 211-222.

Sharp RE, Silk WK, Hsiao TC. 1988. Growth of the maize primary root at low water potential. I. Spatial distribution of expansive growth. Plant Physiology 87: 50-57.

Sharp RE and Davies WJ. 1979. Solute Regulation and Growth by Roots and Shoots of Water-Stressed Maize Plants. Planta 147: 43-49.

Silk WK. 1984. Quantitative descriptions of development. Annual Review of Plant Physiology 35:479-518.

Sobeih WY, Dodd IC, Bacon MA, Grierson D, and Davies WJ. 2004. Longdistance signals regulating stomatal conductance and leaf growth in tomato 
(Lycopersicon esculentum) plants subjected to partial root-zone drying. Journal of Experimental Botany 55 (407): 2353-2363.

Sokal RR and Rohlf FJ. 1995. Biometry: The Principles and Practice of Statistics in Biological Research. Third Edition. WH Freeman and Company.

Spollen WG, LeNoble ME, Samuels TD, Bernstein N, Sharp RE. 2000. Abscisic Acid Accumulation Maintains Maize Primary Root Elongation at Low Water Potentials by Restricting Ethylene Production. Plant Physiology 122: 967976.

Spollen WG, Sharp RE, Saab IN, Wu Y. 1993. Regulation of cell expansion in roots and shoots at low water potentials. In Water Deficits: Plant Responses from Cell to Community (eds J.A.C.Smith, H.Griffiths) pp. 3752. BIOS Scientific Publishers, Oxford, UK.

Steudle E. 2000. Water uptake by plant roots: an integration of views. Plant and Soil 226: 45-56.

Steudle E, Peterson CA. 1998. How does water get through roots? Journal of Experimental Botany 49: 775-788.

Steudle E, Oren R, Schulze ED. 1987. Measurement of hydraulic conductivity, solute permeability, and of reflection coefficients of excised roots using the root pressure probe. Plant Physiology 84(4): 1220-1232.

Tardieu F, Davies WJ. 1992. Stomatal response to abscisic acid is a function of current plant water status. Plant Physiology 98: 540-545. 
Taiz L and Zeiger E. 2010. Plant Physiology, Fifth Edition, published by Sinauer Associates. Sunderland, MA.

Tezara W, Mitchell VJ, Driscoll SD, Lawlor DW. 1999. Water stress inhibits plant photosynthesis by decreasing coupling factor and ATP. Nature 401:914917.

The Royal Society. 2009. Reaping the benefits: Science and the sustainable intensification of global agriculture. The Royal Society Policy Document 11/09, The Royal Society, London.

Thompson AJ, Andrews J, Mulholland BJ, McKee JM, Hilton HW, Horridge JS, Farquhar GD, Smeeton RC, Smillie IR, Black CR, et al. 2007. Overproduction of abscisic acid in tomato increases transpiration efficiency and root hydraulic conductivity and influences leaf expansion. Plant Physiology 143: 1905-1917.

Tolivia D, Tolivia J. 1987. Fasga: a new polychromatic method for simultaneous and differential staining of plant tissues. Journal of Microscopy 148:113117.

USDA. 2016. Crops: Corn. Economic Research Service. http://www.ers.usda.gov/topics/crops/corn/

Verslues PE, Ober ES, Sharp RE. 1998. Root growth and oxygen relations at low water potentials: impact of oxygen availability in polyethylene glycol solutions. Plant Physiology 116: 1403-1412. 
Volkmar KM. 1997.Water stressed nodal roots of wheat: effects on leaf growth. Australian Journal of Plant Physiology 24: 49-56.

Voothuluru, P., Anderson, J. C., Sharp, R. E., and Peck, S. C. 2016. Plasma membrane proteomics in the maize primary root growth zone: novel insights into root growth adaptation to water stress. Plant, Cell and Environment 39: 2043-2054.

Voothuluru P., Sharp R.E. 2013. Apoplastic hydrogen peroxide in the growth zone of the maize primary root under water stress. I. Increased levels are specific to the apical region of growth maintenance. Journal of Experimental Botany 64, 1223-1233.

Wallander S, Aillery M, Hellerstein D, and Hand M. 2013. The Role of Conservation Programs in Drought Risk Adaptation. Washington, DC: U.S. Department of Agriculture, Economic Research Service, Economic Research Report No. 148.

Wang XL, Canny MJ, McCully ME. 1991. The water status of the roots of soilgrown maize in relation to the maturity of their xylem. Physiologia Planatarum. 82: 157-162.

Weaver JE. 1926. Root Development of Field Crops. New York-London: McGraw-Hill Book Co.

Wenzel CL, McCully ME, Canny MJ. 1989. Development of water conducting capacity in the root systems of young plants of corn and some other C4 grasses. Plant Physiology 89: 1094-1101. 
Westgate ME, Boyer JS. 1985. Osmotic adjustment and the inhibition of leaf, root, stem and silk growth at low water potentials in maize. Planta 164: 540-549.

Wiegers BS, Cheer AY, Silk WK. 2009. Modeling the hydraulics of root growth in three dimensions with phloem water sources. Plant Physiology 150: 20922103.

Yamaguchi M., Sharp R.E. 2010. Complexity and coordination of root growth at low water potentials: recent advances from transcriptomic and proteomic analyses. Plant, Cell and Environment 33: 590-603.

Zhang J, Davies WJ. 1991. Antitranspirant activity in xylem sap of maize plants. Journal of Experimental Botany 42: 317-321.

Zhang Z, Voothuluru P, Yamaguchi M, Sharp RE, Peck SC. 2013.

Developmental distribution of the plasma membrane-enriched proteome in the maize primary root growth zone. Frontiers in Plant Science 4: 33. 


\section{VITA}

Kara J. Riggs was born in February, 1988 in Minnesota. She received her Bachelor of Science in Agriculture from The Ohio State University in 2010. Her interest in food security began when she read about golden rice in 2000 , and she has followed this interest into researching methods of crop improvement. She joined the Division of Plant Sciences, University of Missouri, Columbia in August 2010. Her Ph.D. research is focused on root growth maintenance of maize under water deficit conditions. 